\title{
Nitro-Substituted Hoveyda-Grubbs Ruthenium Carbenes: Enhancement of Catalysts Activity through Electronic Activation
}

\author{
Supplementary Information
}

\author{
Anna Michrowska, ${ }^{a}$ Robert Bujok, ${ }^{a}$ Syuzanna Harutyunyan, ${ }^{a}$ Volodymyr \\ Sashuk, ${ }^{a}$ Grigory Dolgonos ${ }^{b}$ and Karol Grela ${ }^{*, a}$ \\ a Institute of Organic Chemistry, Polish Academy of Sciences, Kasprzaka 44/52, \\ 01-224 Warsaw, Poland; ${ }^{b}$ Institute of Physical Chemistry, Polish Academy of \\ Sciences, Kasprzaka 44/52, 01-224 Warsaw, Poland \\ grela@icho.edu.pl
}

\section{General}

Unless otherwise noted, all reactions were carried out under Ar in pre-dried glassware using Schlenk techniques. The solvents were dried by distillation over the following drying agents and were transferred under argon: THF (K/benzophenone), toluene $(\mathrm{Na})$, n-pentane, $n$-hexane, $\mathrm{CH}_{2} \mathrm{Cl}_{2}\left(\mathrm{CaH}_{2}\right), \mathrm{Et}_{2} \mathrm{O}$ $\left(\mathrm{LiAlH}_{4}\right), \mathrm{MeOH}(\mathrm{Mg})$. Flash column chromatography: Merck silica gel 60 (230-400 mesh). NMR: Spectra were recorded on Bruker AVANCE 500, Varian Gemini 200 and 400 spectrometers in $\mathrm{CDCl}_{3}$; chemical shifts $(\delta)$ are given in ppm relative to TMS, coupling constants $(J)$ in Hz. IR: Perkin-Elmer Spectrum 2000 FT-IR, wavenumbers in $\mathrm{cm}^{-1}$. MS (EI, LSIMS): AMD 604 Intectra GmbH. MS (ESI): Mariner Perseptive Biosystems, Inc. GC: HP 6890 with HP 5 column. GC/MS: HP 5890 with HP 5 column. Micro-analyses were provided by Institute of Organic Chemistry, PAS, Warsaw. The following catalysts and substrates were prepared according to the literature procedures: $4 \mathbf{b},{ }^{1} \mathbf{2 2},{ }^{2}, \mathbf{2 9},{ }^{3}$ substrates for $\mathbf{3 6}$ and $\mathbf{4 2 - 4 4},{ }^{4} \mathbf{4 5},{ }^{5} \mathbf{5 0}, \mathbf{5 1}$ and $\mathbf{5 3},{ }^{6}$ $55,{ }^{7} 56-57,{ }^{8} \mathbf{7 1} .^{9}$ All commercially available chemicals were used as received.

\section{Synthesis of catalysts $6-10$ and 70}

Alkylation of nitrosalicylaldehydes 11-13. General Procedure: To a suspension of $\mathrm{K}_{2} \mathrm{CO}_{3}(1.11 \mathrm{~g}, 8.0 \mathrm{mmol})$ and $\mathrm{Cs}_{2} \mathrm{CO}_{3}(0.52$ $\mathrm{g}, 40 \mathrm{mmol})$ in DMF $(20 \mathrm{~mL})$ a corresponding nitrosalicylaldehyde $(4.0 \mathrm{mmol})$ was added. After stirring for $10 \mathrm{~min}$ at room temperature, 2-iodopropane $(0.8 \mathrm{~mL}, 1.36 \mathrm{~g}, 8.0 \mathrm{mmol})$ was added and the reaction mixture was stirred for 1-2 days at $40{ }^{\circ} \mathrm{C}$. Then the reaction mixture was poured into $20 \mathrm{~mL}$ water and extracted three times with EtOAc. The combined extracts were washed with brine, water

\footnotetext{
1 Wakamatsu, H.; Blechert, S. Angew. Chem., Int. Ed. Engl. 2002, 41, 2403-2405.

2 Henley, R. V.; Turner, E. E. J. Chem. Soc. 1930, 928 -940

3 Mąkosza, M.; Stalewski, J.; Wojciechowski, K.; Danikiewicz, W. Tetrahedron 1997, 53, $193-214$.

4 Moskalev, N.; Mąkosza, M. Tetrahedron Lett. 1999, 40, 5935; (b) Moskalev, N.; Barbasiewicz, M.; Mąkosza, M. Tetrahedron 2004, 60, 347-358.

5 (a) Pietrusiewicz, K. M.; Zablocka, M.; Monkiewicz, J. J. Org. Chem., 1984, 49, 152; (b) Pietrusiewicz, K. M.; Zabłocka, M. Tetrahedron Lett., 1988, 29, 199.

6 Mąkosza, M.; Nieczypor, P.; Grela, K. Tetrahedron 1998, 54, 10827-10836.

7 Prowotorow, I.; Wicha, J.; Mikami, K. Synthesis 2001, 145-149.

8 Furman, B.; Dziedzic, M. Tetrahedron Lett. 2003, 44, 8249-8252.

9 Grela, K.; Michrowska, A.; Bieniek, M.; Kim, M.; Klajn, R. Tetrahedron, 2003, 59, 4525-4531.
} 
and dried. The solvent was evaporated. The products 11a-13a were used without further purification.

2-isopropoxy-5-nitrobenzaldehyde (11a): Yellow low melting solid $(86 \%)$. IR $(\mathrm{KBr}): \nu$ 3115, 2991, 2942, 1679, 1609, 1526, 1348, 1284, 1111, 950, 832, 748, $667 \mathrm{~cm}^{-1} ;{ }^{1} \mathrm{H}$ NMR (500 $\left.\mathrm{MHz}, \mathrm{CDCl}_{3}\right) \delta 1.48(\mathrm{~d}, 6 \mathrm{H}, J=6.1 \mathrm{~Hz})$, $4.85(\mathrm{q}, 1 \mathrm{H}, J=6.1 \mathrm{~Hz}), 7.10(\mathrm{~d}, 1 \mathrm{H}, J=9.2$ $\mathrm{Hz}), 8.39(\mathrm{dd}, 1 \mathrm{H}, J=2.9,9.2 \mathrm{~Hz}), 8.69(\mathrm{~d}$, $1 \mathrm{H}, J=2.9 \mathrm{~Hz}), 10.41(\mathrm{~s}, 1 \mathrm{H}) ;{ }^{13} \mathrm{C}$ NMR $(125$ $\mathrm{MHz}, \mathrm{CDCl}) \delta 21.8,72.6,113.6,124.7,125.1$, 130.4, 141.1, 164.3, 187.8; MS (EI) $\mathrm{m} / \mathrm{z}$ (rel intensity) $209\left(10,[\mathrm{M}]^{+\cdot}\right), 167(100), 137$ (18), 120 (11), 93 (7), 75 (3), 65 (10), 53 (4); HRMS (EI) calcd for $[\mathrm{M}]^{+\cdot}\left(\mathrm{C}_{10} \mathrm{H}_{11} \mathrm{O}_{4} \mathrm{~N}\right): 209.0688$. found 209.0686 .

2-isopropoxy-3-nitrobenzaldehyde (12a): Brown oil (56\%). ${ }^{1} \mathrm{H}$ NMR $\left(400 \mathrm{MHz}, \mathrm{CDCl}_{3}\right)$ $\delta 1.88(\mathrm{~d}, 6 \mathrm{H}, J=6.3 \mathrm{~Hz}), 4.34$ (heptet, $1 \mathrm{H}$, $J=6.2 \mathrm{~Hz}), 7.31-7.36(\mathrm{~m}, 1 \mathrm{H}), 8.07-8.12(\mathrm{~m}$, $2 \mathrm{H}), 10.42(\mathrm{~d}, 1 \mathrm{H}, J=0.9 \mathrm{~Hz}) ; \mathrm{MS}(\mathrm{ESI})$ $\mathrm{m} / z$ (rel intensity) $264.1\left([\mathrm{M}+\mathrm{H}+2 \mathrm{MeOH}]^{+}\right)$, $232.1\left([\mathrm{M}+\mathrm{H}+\mathrm{MeOH}]^{+}\right)$.

2-isopropoxy-3-methoxy-5-nitrobenzaldehyde (13a): yellow-brown crystals (71\%). MS (LSIMS) $\mathrm{m} / \mathrm{z}$ (rel intensity) 240 $\left([\mathrm{M}+\mathrm{H}]^{+}\right)$; HRMS (LSIMS) calcd for $[\mathrm{M}+\mathrm{H}]^{+}$ $\left(\mathrm{C}_{11} \mathrm{H}_{14} \mathrm{O}_{5} \mathrm{~N}\right)$ : 240.08720; found: 240.08661 .

General procedure for Wittig olefination: In a three-necked flame-dried flask solid methyltriphenylphosphonium bromide (0.384 g, $1.1 \mathrm{mmol}$, Aldrich) and THF (3.5 $\mathrm{mL}$ ) were placed under argon. Then a solution of $n$-BuLi $(1 \mathrm{~mL}, 1.5 \mathrm{mmol}, 1.5 \mathrm{M})$ was added dropwise at $-78{ }^{\circ} \mathrm{C}$. After stirring 15 min at $-78{ }^{\circ} \mathrm{C}$, a solution of corresponding 2-isopropoxybenzaldehyde 11a-13a (0.75 $\mathrm{mmol})$ in THF $(1 \mathrm{~mL})$ was added and the reaction mixture was stirred at the same temperature for $30 \mathrm{~min}$. After warming to RT the reaction mixture was stirred for $48 \mathrm{~h}$. The reaction mixture was treated with saturated solution of $\mathrm{NH}_{4} \mathrm{Cl}$, extracted with EtOAc and dried. The solvent was evaporated and the product was purified by column chromatography (c-hexane-ethyl acetate $2: 8$ ).

2-isopropoxy-5-nitrostyrene (14): Pale yellow oil (57\%). IR (film): $\nu$ 3088, 2982, 2967, 1627, 1607, 1583, 1516, 1341, 1271, 1107, 950, $742 \mathrm{~cm}^{-1} ;{ }^{1} \mathrm{H}$ NMR $(500 \mathrm{MHz}, \mathrm{CDCl}) \delta 1.41$ $(\mathrm{d}, 6 \mathrm{H}, J=6.0 \mathrm{~Hz}), 4.71(\mathrm{q}, 1 \mathrm{H}, J=6.0 \mathrm{~Hz})$, $5.40(\mathrm{dd}, 1 \mathrm{H}, J=0.5,11.2 \mathrm{~Hz}), 5.87(\mathrm{dd}, 1 \mathrm{H}$, $J=0.5,17.7 \mathrm{~Hz}), 6.91(\mathrm{~d}, 1 \mathrm{H}, J=9.1 \mathrm{~Hz})$, $7.00(\mathrm{dd}, 1 \mathrm{H}, J=11.2,17.7 \mathrm{~Hz}), 8.12(\mathrm{dd}, 1 \mathrm{H}$, $J=2.8,9.1 \mathrm{~Hz}), 8.36(\mathrm{~d}, 1 \mathrm{H}, J=2.8 \mathrm{~Hz}) ;{ }^{13} \mathrm{C}$ NMR $(125 \mathrm{MHz}, \mathrm{CDCl}) \delta 21.9,71.5,112.2$, 116.8, 122.4, 124.5, 128.1, 130.1, 141.0, 159.9; MS (EI) $\mathrm{m} / z$ (rel intensity) $207\left(4,[\mathrm{M}]^{+\cdot}\right), 165$ (59), 148 (100), 135 (4), 118 (96), 104 (2), 90 (15), 65 (8), 63 (7), 51 (4); MS (ESI): $m / z 230$ $\left([\mathrm{M}+\mathrm{Na}]^{+}\right)$; HRMS (ESI) calcd for $[\mathrm{M}+\mathrm{Na}]^{+}$ $\left(\mathrm{C}_{11} \mathrm{H}_{13} \mathrm{O}_{3} \mathrm{NNa}\right)$ : 230.0788. found 230.0776.

2-isopropoxy-3-nitrostyrene (15): Brown oil $(67 \%) .{ }^{1} \mathrm{H}$ NMR $\left(200 \mathrm{MHz}, \mathrm{CDCl}_{3}\right) \delta$ $1.28(\mathrm{~d}, 6 \mathrm{H}, J=6.0 \mathrm{~Hz}), 4.23$ (heptet, $1 \mathrm{H}, J$ $=6.2 \mathrm{~Hz}), 5.43(\mathrm{dd}, 1 \mathrm{H}, J=11.0,1.0 \mathrm{~Hz})$, $5.80(\mathrm{dd}, 1 \mathrm{H}, J=17.8,1.0 \mathrm{~Hz}), 6.95-7.28$ $(\mathrm{m}, 2 \mathrm{H}), 7.65-7.74(\mathrm{~m}, 2 \mathrm{H}) ;{ }^{13} \mathrm{C}$ NMR $(50$ $\left.\mathrm{MHz}, \mathrm{CDCl}_{3}\right) \delta 148.2,134.8,130.7,130.4$, 124.1, 123.4, 117.0, 78.9, 22.1; MS (EI) $\mathrm{m} / z$ (rel intensity) $207\left(4,[\mathrm{M}]^{+\cdot}\right), 165(59), 149$ (10), 148 (100), 90 (15), 89 (14); HRMS (EI) calcd for $[\mathrm{M}]^{+\cdot}\left(\mathrm{C}_{11} \mathrm{H}_{13} \mathrm{O}_{3} \mathrm{~N}\right)$ : 207.08954; found: 207.09005

\section{2-isopropoxy-3-methoxy-5-nitrostyrene} (16): Brown oil (63\%). IR (film): $\nu$ 3095, 2978, 2937, 2872, 2664, 1694, 1612, 1580, 1524, $1465,1430,1373,1340,1300,1282,1226,1185$, $1141,1099,1073,1048,996,928,885,852,802$, 776, 747, 576, $463 \mathrm{~cm}^{-1}$; ${ }^{1} \mathrm{H}$ NMR (500 MHz, acetone- $\left.d_{6}\right) \delta 1.30(\mathrm{~d}, 6 \mathrm{H}, J=6.1 \mathrm{~Hz}), 3.93$ $(\mathrm{s}, 3 \mathrm{H}), 4.63$ (heptet, $1 \mathrm{H}, J=6.1 \mathrm{~Hz}$ ), 5.41 $(\mathrm{dd}, 1 \mathrm{H}, J=11.1,1.0 \mathrm{~Hz}), 5.84(\mathrm{dd}, 1 \mathrm{H}, J=$ 17.7, $1.0 \mathrm{~Hz}) 7.03-7.09(\mathrm{~m}, 1 \mathrm{H}) 7.67(\mathrm{~d}, 1 \mathrm{H}$, $J=2.6 \mathrm{~Hz}), 8.07(\mathrm{~d}, 1 \mathrm{H}, J=2.6 \mathrm{~Hz}) ;{ }^{13} \mathrm{C}$ NMR $\left(125 \mathrm{MHz}\right.$, acetone- $\left.d_{6}\right) \delta 153.2,150.0$, 143.5, 132.8, 130.5, 117.0, 113.8, 106.1, 76.4, $56.2,22.5$; MS (EI) $m / z$ (rel intensity) 238 (2), $237\left(11, \mathrm{M}^{+\cdot}\right), 196$ (10), 195 (100), 134 (9), 43 (9); HRMS (EI) calcd for [M] ${ }^{+\cdot}\left(\mathrm{C}_{12} \mathrm{H}_{15} \mathrm{O}_{4} \mathrm{~N}\right)$ : 237.10011; found: 237.09979 .

3,5-dibromobiphenyl-2-ol (18): To a solution of 7 (4.25 g, 25 mmole) in $\mathrm{AcOH}(5 \mathrm{~mL})$, $\mathrm{Br}_{2}$ (3 mL, $9.33 \mathrm{~g}$, $58 \mathrm{mmole}$ ) was added at RT in portions-the reaction is exothermic in initial stage and the flask was cooled in cold water when necessary. The mixture was stirred at RT for $2 \mathrm{~h}$, then poured into water $(80 \mathrm{~mL})$ and solid $\mathrm{K}_{2} \mathrm{CO}_{3}$ was added in portions to $\mathrm{pH} 5$. The mixture was extracted with MTBE $(3 \times 50 \mathrm{~mL})$, the combined extracts were washed with aqueous $\mathrm{Na}_{2} \mathrm{SO}_{3}$, dried and evaporated. The product (7.56 g, $92 \%)$ was used without further purification. 
Yellowish solid, $\mathrm{mp}=52-55{ }^{\circ} \mathrm{C}$; lit.: ${ }^{10} 56-57$ ${ }^{\circ} \mathrm{C}$.

3-bromo-5-nitrobiphenyl-2-ol (19): To a suspension of $18(0.64 \mathrm{~g}, 1.9 \mathrm{mmol})$ in $\mathrm{AcOH}$ $(0.8 \mathrm{~mL})$ concentrated $\mathrm{HNO}_{3}(0.15 \mathrm{~mL}, 2.1$ mmol) was added slowly ( 3 minutes) at $5{ }^{\circ} \mathrm{C}$ and the mixture was stirred for additional 5 min. Water $(25 \mathrm{~mL})$ was then added and the resulted mixture was extracted with $\mathrm{CH}_{2} \mathrm{Cl}_{2}$ ( $3 \times 25 \mathrm{~mL})$. The combined extracts were washed with water $(3 \times 25 \mathrm{~mL})$, dried and evaporated to dryness to give $0.216 \mathrm{~g}(39 \%)$ of the product as a orange-brown solid. mp $=111-113{ }^{\circ} \mathrm{C}$, lit.: $:^{11} 113.5-114{ }^{\circ} \mathrm{C}$.

\section{2-isopropoxy-3-bromo-5-nitrobiphenyl} (19a): The compound was obtained according to procedure used for alkylation of the nitrosalicylaldehydes 11-13. Yellow oil (70\%). IR (film): $\nu$ 3081, 2979, 2932, 2871, 1602, 1581, 1567, 1523, 1497, 1464, 1452, 1429, 1384, 1373, 1337, 1288, 1237, 1175, 1141, 1099, 1054, 924, 904, 850, 809, 782, 768, 743, 701, 634, 566, $510,437 \mathrm{~cm}^{-1} ;{ }^{1} \mathrm{H}$ NMR $\left(400 \mathrm{MHz}, \mathrm{CDCl}_{3}\right)$ $\delta 1.06(\mathrm{~d}, 6 \mathrm{H}, J=6.2 \mathrm{~Hz}), 4.06$ (heptet, $1 \mathrm{H}$, $J=6.1 \mathrm{~Hz}), 7.42-7.50(\mathrm{~m}, 3 \mathrm{H}), 7.54-7.57(\mathrm{~m}$, $2 \mathrm{H}), 8.18(\mathrm{~d}, 1 \mathrm{H}, \mathrm{J}=2.7 \mathrm{~Hz}) 8.44(\mathrm{~d}, 1 \mathrm{H}, J=$ $2.9 \mathrm{~Hz}$ ); MS (EI) $m / z$ (rel intensity) 337 (4), $335\left(4,[\mathrm{M}]^{+\cdot}\right), 296$ (13), 295 (99), 293 (100), 168 (31) 140 (9), 139 (24), 43 (10).

\section{2-Isopropoxy-5-nitro-3-vinylbiphenyl}

(20): In a Schlenk flask was placed solid $\mathrm{Pd}\left[\mathrm{Ph}_{3} \mathrm{P}\right]_{4}(0.015 \mathrm{~g}, 5 \mathrm{~mol} \%)$ and a solution of 19a $(0.088 \mathrm{~g}, 0.26 \mathrm{mmol})$ in toluene $(1.0 \mathrm{~mL})$ was added under argon. Neat $\mathrm{Bu}_{3} \mathrm{SnCH}=\mathrm{CH}_{2}(0.093 \mathrm{~g}, 0.29 \mathrm{mmol})$ was added via syringe and the resulting solution was heated at $115{ }^{\circ} \mathrm{C}$ for $6 \mathrm{~h}$. After cooling to RT the mixture was evaporated and a solution of KF $(0.58 \mathrm{~g})$ in methanol $(5 \mathrm{~mL})$ were added to the residue. The mixture was stirred at RT for $2 \mathrm{~h}$ and evaporated again. To the resulting residue MTBE $(15 \mathrm{~mL})$ and water $(10 \mathrm{~mL})$ were added. Organic phase was removed, inorganic phase was extracted with MTBE $(4 \times 15 \mathrm{~mL})$. The combined extracts were dried and evaporated. The product was purified by column chromatography (c-hexane-ethyl acetate $1.5: 8$ ) to give 0.059 g $(78 \%)$ of 20 as a yellow oil. IR (film): $\nu$ 3085, 3030, 2976, 2929, 2871, 2854, 1734, 1628, $1603,1584,1525,1497,1464,1453,1430,1408$, $1384,1341,1302,1282,1229,1219,1176,1140$,
$1102,1083,1029,996,939,922,858,816,775$, 762, 747, 721, 699, 653, 622, 551, 511, 462, 435 $\mathrm{cm}^{-1} ;{ }^{1} \mathrm{H}$ NMR $\left(400 \mathrm{MHz}, \mathrm{CDCl}_{3}\right) \delta 1.00(\mathrm{~d}$, $6 \mathrm{H}, J=6.2 \mathrm{~Hz}$ ), 3.86 (heptet, $1 \mathrm{H}, J=6.2 \mathrm{~Hz}$ ), $5.47(\mathrm{dd}, 1 \mathrm{H}, \mathrm{J}=11.1,0.8 \mathrm{~Hz}), 5.90(\mathrm{dd}, 1 \mathrm{H}$, $J=17.7,0.8 \mathrm{~Hz}) 7.12(\mathrm{dd}, 1 \mathrm{H}, J=17.8,11.1$ $\mathrm{Hz}) 7.38-7.48$ (m, 3H), 7.55-7.58 (m, 2H), 8.13 $(\mathrm{d}, 1 \mathrm{H}, J=2.9 \mathrm{~Hz}) 8.39(\mathrm{~d}, 1 \mathrm{H}, J=2.8 \mathrm{~Hz})$; ${ }^{13} \mathrm{C}$ NMR $\left(100 \mathrm{MHz}, \mathrm{CDCl}_{3}\right) \delta$ 158.0, 143.7, $137.3,136.8,134.0,131.0,128.9,128.5,128.1$, 125.4, 120.4, 117.2, 76.8, 22.0; MS (EI) $\mathrm{m} / z$ (rel intensity) $284(3), 283\left(15,[\mathrm{M}]^{+\cdot}\right), 242(16)$, 241 (100), 224 (37), 195 (9), 194 (29), 165 (19), 152 (11), 43 (9); HRMS (EI) calcd for [M] ${ }^{+}$. $\left(\mathrm{C}_{17} \mathrm{H}_{17} \mathrm{O}_{3} \mathrm{~N}\right)$ : 283.12084; found: 283.12106 .

\section{1-Bromo-2-isopropoxy-4-nitrobenzene} (22a): The compound was obtained according to procedure used for alkylation of the nitrosalicylaldehydes 11-13. Yellowish solid, $\mathrm{mp}=38-40{ }^{\circ} \mathrm{C}$. IR (film): $\nu$ 3104, 2983, 2938, 1897, 1755, 1566, 1531, 1467, 1410, 1351, $1310,1262,1179,1139,1124,1106,1035,979$, $878,848,821,799,740,701,607,502,470,441$ $\mathrm{cm}^{-1} ;{ }^{1} \mathrm{H}$ NMR $\left(400 \mathrm{MHz}, \mathrm{CDCl}_{3}\right) \delta 1.44$ $(\mathrm{d}, 6 \mathrm{H}, J=6.0 \mathrm{~Hz}), 4.70$ (heptet, $1 \mathrm{H}, J=6.0$ $\mathrm{Hz})$, 7.69-7.70 (m, 2H), 7.72-7.73 (m, 1H); ${ }^{13} \mathrm{C}$ NMR $\left(100 \mathrm{MHz}, \mathrm{CDCl}_{3}\right) \delta$ 155.0, 147.9, 133.6, 121.1, 116.2, 108.4, 72.8, 21.7; MS (EI) $m / z$ (rel intensity) $261(11), 259\left(11,[\mathrm{M}]^{+\cdot}\right), 219$ (97), 217 (100), 189 (13), 187 (14), 173 (16), 171 (16), 161 (19), 159 (20), 145 (13), 143 (14), 92 (18), 79 (13), 75 (12), 63 (54), 62 (13), 43 (77), 41 (39), 39 (15).

2-Isopropoxy-4-nitrostyrene (23) In a Schlenk flask was placed solid $\mathrm{Pd}\left[\mathrm{Ph}_{3} \mathrm{P}\right]_{4}$ $(0.069 \mathrm{~g}, 3 \mathrm{~mol} \%)$ and a solution of $22 \mathrm{a}(0.517$ $\mathrm{g}, 2.0 \mathrm{mmol})$ in toluene $(8 \mathrm{~mL})$ was added under argon. Neat $\mathrm{Bu}_{3} \mathrm{SnCH}=\mathrm{CH}_{2}(0.692 \mathrm{~g}$, $2.2 \mathrm{mmol}$ ) was added via syringe and the resulting solution was refluxed for $6 \mathrm{~h}$. After cooling to RT the mixture was evaporated and to the residue a solution of KF $(0.7 \mathrm{~g})$ in methanol $(10 \mathrm{~mL})$ were added. The mixture was stirred at RT for $2 \mathrm{~h}$ and evaporated again. To the residue MTBE $(30 \mathrm{~mL})$ and water $(15 \mathrm{~mL})$ were added. Organic phase was removed, inorganic phase (containing insoluble material) was extracted with MTBE (3 x $25 \mathrm{~mL}$ ) and combined extracts were dried and evaporated. The product was purified by column chromatography (c-hexane-ethyl acetate $2: 18)$ to give $0.343 \mathrm{~g}(83 \%)$ of 23 as a yellow oil. IR (film): $\nu$ 3091, 2980, 2933,

\footnotetext{
10 Auwers, V.; Wittig, G. J. Prakt. Chem. 1924, 108, 103.

11 Hartshorn, M. P.; Robinson, W. T.; Vaughan, J.; White, J. M. Austr. J. Chem. 1985, 38, 575.
} 
1694, 1626, 1584, 1520, 1486, 1417, 1345, 1293, 1248, 1179, 1111, 981, 916, 870, 846, 831, 801, $737 \mathrm{~cm}^{-1} ;{ }^{1} \mathrm{H}$ NMR $\left(400 \mathrm{MHz}, \mathrm{CDCl}_{3}\right) \delta$ $1.41(\mathrm{~d}, 6 \mathrm{H}, J=6.1 \mathrm{~Hz}), 4.68$ (heptet, $1 \mathrm{H}, J$ $=6.0 \mathrm{~Hz}), 5.46(\mathrm{dd}, 1 \mathrm{H}, J=11.3,1.2 \mathrm{~Hz})$, $5.90(\mathrm{dd}, 1 \mathrm{H}, J=17.8,1.1 \mathrm{~Hz}) 7.02-7.10(\mathrm{~m}$, $1 \mathrm{H}), 7.58(\mathrm{~d}, 1 \mathrm{H}, J=8.6 \mathrm{~Hz}), 7.72(\mathrm{~d}, 1 \mathrm{H}$, $J=3.2 \mathrm{~Hz}), 7.77-7.80(\mathrm{~m}, 1 \mathrm{H}) ;{ }^{13} \mathrm{C}$ NMR $\left(100 \mathrm{MHz}, \mathrm{CDCl}_{3}\right) \delta 155.1,147.8,134.1,130.5$, 126.6, 118.2, 115.6, 108.2, 71.4, 21.8; MS (EI) $\mathrm{m} / \mathrm{z}$ (rel intensity) $208(4), 207\left(22,[\mathrm{M}]^{+\cdot}\right), 165$ (100), 135 (20), 119 (10), 118 (13), 107 (16), 91 (38), 89 (18), 79 (14), 65 (19), 63 (10), 43 (32), 41 (19), 39 (13); HRMS (EI) calcd for [M] ${ }^{+}$ $\left(\mathrm{C}_{11} \mathrm{H}_{13} \mathrm{O}_{3} \mathrm{~N}\right)$ : 207.08954; found: 207.08872; Anal. Calcd. for $\mathrm{C}_{11} \mathrm{H}_{13} \mathrm{O}_{3} \mathrm{~N}: \mathrm{C}, 63.76 ; \mathrm{H}$, 6.32; $\mathrm{N}, 6.76$. found: $\mathrm{C}, 63.54 ; \mathrm{H}, 6.49 ; \mathrm{N}, 6.61$.

2-methoxy-5-nitrostyrene (70a): Solid $\mathrm{Ph}_{3} \mathrm{P}=\mathrm{CH}_{2} \quad(0.693 \mathrm{~g}, 2.5 \mathrm{mmol}$, Aldrich) and THF $(20 \mathrm{~mL})$ were placed under argon in a Schlenk tube. A solution of 2-methoxy-5-nitrobenzaldehyde $(0.202 \mathrm{~g}, 1.1$ mmol, Aldrich) in THF (5 mL) was added at $-78{ }^{\circ} \mathrm{C}$. The reaction mixture was stirred at the same temperature for $1 \mathrm{~h}$. Then the reaction mixture was warmed to $\mathrm{RT}$ and treated with saturated solution of $\mathrm{NH}_{4} \mathrm{Cl}$, extracted with MTBE $(4 \times 20 \mathrm{~mL})$ and dried. The solvent was evaporated and the product was purified by column chromatography to give $0.115 \mathrm{~g}(58 \%)$ of the product as a yellow oil. IR (KBr): $\nu$ 3115, 3090, 3023, 2962, 2924, 2851, 2655, 2513, 2247, 2018, 1902, 1847, 1805, $1779,1731,1624,1610,1583,1515,1467,1458$, $1445,1421,1339,1257,1186,1141,1085,1018$, $992,918,903,827,802,759,743,637,597,520$ $504,433 \mathrm{~cm}^{-1} ;{ }^{1} \mathrm{H}$ NMR $\left(500 \mathrm{MHz}, \mathrm{CDCl}_{3}\right) \delta$ $3.96(\mathrm{~s}, 3 \mathrm{H}), 5.41(\mathrm{dd}, 1 \mathrm{H}, J=11.2,0.9 \mathrm{~Hz})$, $5.86(\mathrm{dd}, 1 \mathrm{H}, J=17.7,1.0 \mathrm{~Hz}), 6.91-7.02(\mathrm{~m}$, $2 \mathrm{H}), 8.14(\mathrm{dd}, 1 \mathrm{H}, J=9.1,2.8 \mathrm{~Hz}), 8.34(\mathrm{~d}$, $1 \mathrm{H}, J=2.8 \mathrm{~Hz}) ;{ }^{13} \mathrm{C}$ NMR $\left(125 \mathrm{MHz}, \mathrm{CDCl}_{3}\right)$ $\delta 161.3,141.5,129.8,127.6,124.7,122.1,117.2$, 110.4, 56.1; MS (EI) $\mathrm{m} / \mathrm{z}$ (rel intensity) 180 (8), $179\left(68,[\mathrm{M}]^{+*}\right), 164(46), 149$ (9), $118(100)$, 103 (14), 90 (33), 89 (35), 79 (11), 77 (30), 63 (17), 51 (11); HRMS (EI) calcd for [M] ${ }^{+}$. $\left(\mathrm{C}_{19} \mathrm{H}_{9} \mathrm{O}_{3} \mathrm{~N}\right)$ : 179.05824 ; found: 179.05780 .

\subsection{General procedure for preparation of catalysts $6 a$ and $7 a$}

Catalyst 6a: Under an argon atmosphere Grubbs' catalyst 1a $(164.6 \mathrm{mg}, 0.20 \mathrm{mmol})$ was placed in a Schlenk tube. Then $\mathrm{CH}_{2} \mathrm{Cl}_{2}$ $(15 \mathrm{~mL})$ was added followed by a solution of styrene 14 in $\mathrm{CH}_{2} \mathrm{Cl}_{2}(5 \mathrm{~mL})$. The resulted suspension was stirred at $40{ }^{\circ} \mathrm{Cfor} 1 \mathrm{~h}$. After this point forth all manipulations can be done without a protective atmosphere of argon. The resulting mixture was concentrated in vacuo, and purified by silica-gel column chromatography, using c-hexane-EtOAc (5:2) as eluent and collecting a product as a brown band. After removal of solvents and washing with small amount of dry n-pentane 6a was obtained as brown, microcrystalline solid (95 mg, 83\%). IR (solid film): $\nu$ 2930, 2852, 1604, 1575, 1521, 1476, 1447, 1379, 1342, 1275, 1241, 1205, 1181, 1136, 1095, 1049, 1005, 951, 918, $851,830,789,745,656,606,518 \mathrm{~cm}^{-1} ;{ }^{1} \mathrm{H}$ NMR $\left(500 \mathrm{MHz}, \mathrm{CDCl}_{3}\right) \delta$ 1.26-2.35 (m, 39 $\mathrm{H}), 5.33-5.40(\mathrm{~m}, 1 \mathrm{H}), 7.18(\mathrm{~d}, 1 \mathrm{H}, J=5 \mathrm{~Hz})$, $8.54(\mathrm{~d}, 1 \mathrm{H}, J=5 \mathrm{~Hz}), 8.60(\mathrm{~s}, 1 \mathrm{H}), 17.38(\mathrm{~d}$, $1 \mathrm{H}, J=5.0 \mathrm{~Hz}) ;{ }^{13} \mathrm{C}$ NMR $\left(125 \mathrm{MHz}, \mathrm{CDCl}_{3}\right)$ $\delta$ 273.2, 157.0, 143.3, 124.2, 117.6, 113.2, 78.2, $35.8(\mathrm{~d}, J=10 \mathrm{~Hz}), 30.1,27.7(\mathrm{~d}, J=24 \mathrm{~Hz})$, 26.2, 22.1; MS (EI) $\mathrm{m} / \mathrm{z}$ (rel intensity) 645 (9, $\left.[\mathrm{M}]^{+\cdot}\right), 569$ (4), $567(6), 566(4), 419(4), 418$ (3), 417 (8), 416 (10), 415 (10), 414 (14), 413 (10), 412 (10), 411 (7), 410 (6), 409 (4), 408 (5), 379 (7), 378 (5), 377 (13), 376 (11), 375 (11), 374 (10), 373 (6), 372 (4), 371 (4), 285 (14), 281 (13), 280 (10), 279 (10), 215 (15), 214 (69), 213 (10), 199 (24), 198 (43), 167 (12), 159 (10), 153 (18), 133 (69), 132 (57), 123 (10), 117 (100), 116 (27), 115 (23), 113 (11), 83 (50), 82 (18), 81 (37), 79 (18), 78 (16), 77 (15), 67 (18), 55 (74), 54 (11), 43 (70), 42 (16), 41 (77); HRMS (EI): calcd. for $[\mathrm{M}]^{+\cdot}\left(\mathrm{C}_{28} \mathrm{H}_{44} \mathrm{O}_{3} \mathrm{~N}^{35} \mathrm{Cl}_{2} \mathrm{P}^{102} \mathrm{Ru}\right): \quad 645.14794$. found: 645.14706 .

Catalyst 7a: brown solid (70\%) IR (solid film): $\nu$ 2930, 2853, 1605, 1575, 1521, 1447, $1342,1275,1242,1205,1181,1135,1095,1049$, 1005, 951, 918, 851, 830, 789, 745, 656, 606, $518 \mathrm{~cm}^{-1} ;{ }^{1} \mathrm{H}$ NMR $\left(500 \mathrm{MHz}, \mathrm{CDCl}_{3}\right) \delta$ $1.27-2.34(\mathrm{~m}, 39 \mathrm{H}), 5.35-5.40(\mathrm{~m}, 1 \mathrm{H}), 7.84$ (s, 1H), 7.92-7.99 (m, 2H), $17.42(\mathrm{~d}, 1 \mathrm{H}, J=$ $4.2 \mathrm{~Hz}) ;{ }^{13} \mathrm{C}$ NMR $\left(125 \mathrm{MHz}, \mathrm{CDCl}_{3}\right) \delta 271.1$, 152.2, 146.7, 145.7, 121.9, 119.1, 108.6, 77.5, 35.9, $35.7(\mathrm{~d}, J=10 \mathrm{~Hz}), 30.127 .7(\mathrm{~d}, J=24$ $\mathrm{Hz}$ ), 26.9, 26.2, 22.1; MS (ESI) $\mathrm{m} / \mathrm{z}$ (rel intensity) $675.4\left([\mathrm{M}+\mathrm{MeOH}]^{+}\right)$. MS (EI) $\mathrm{m} / z$ (rel intensity) $645\left(3,[\mathrm{M}]^{+\cdot}\right), 296(10), 280(10)$, 241 (12), 215 (24), 214 (100), 213 (17), 207 (24), 199 (29), 198 (39), 180 (13), 179 (29), 178 (11), 177 (12), 167 (23), 166 (15), 165 (82), 162 (15), 159 (16), 153 (20), 149 (11), 135 (18), 133 (88), 132 (63), 131 (13), 118 (10), 117 (67), 116 (16), 115 (18), 113 (11), 107 (11), 105 (11), 97 (10), 95 (11), 91 (29), 89 (10), 83 (44), 82 (18), 81 (36), 79 (21), 78 (18), 77 (20), 71 (13), 69 (17), 67 
(17), 65 (14), 57 (19), 55 (57), 43 (65), 42 (11), 41 (63), 39 (21), 36 (10); HRMS (EI): calcd. for $[\mathrm{M}]^{+\cdot}\left(\mathrm{C}_{28} \mathrm{H}_{44} \mathrm{O}_{3}{ }^{35} \mathrm{Cl}_{2} \mathrm{NP}^{102} \mathrm{Ru}\right): \quad 645.14794$ found 645.14957 .

\subsection{General procedure for preparation of catalysts $6 b, 7 b, 10$ and 70 from $1 b$}

Catalyst 6b: Carbene complex $\mathbf{1 b}(153 \mathrm{mg}$, $0.18 \mathrm{mmol}), \mathrm{CuCl}(18 \mathrm{mg}, 0.18 \mathrm{mmol})$ and $\mathrm{CH}_{2} \mathrm{Cl}_{2}(10 \mathrm{~mL})$ were placed in a Schlenk flask. A solution of styrene $14(38 \mathrm{mg}, 0.18$ mmol) in $\mathrm{CH}_{2} \mathrm{Cl}_{2}(4 \mathrm{~mL})$ was then added and the resulted solution was stirred under $\operatorname{argon}$ at $30{ }^{\circ} \mathrm{C}$ for $1 \mathrm{~h}$. From this point forth, all manipulations were carried out in air with reagent-grade solvents. The reaction mixture was concentrated in vacuo and the resulted material was purified by column chromatography on silica. Elution with $c$-hexane-EtOAc $(5: 2)$ removes $\mathbf{6} \mathbf{b}$ as a green band. Removal of solvent, washing with cold $n$-pentane and drying under vacuum afforded $\mathbf{6 b}$ as a green microcrystalline solid (100 mg, 83\%). IR (KBr): $\nu$ 2924, 2850, 1606, 1521, 1480, 1262, 1093, 918, $745 \mathrm{~cm}^{-1}$; ${ }^{1} \mathrm{H}$ NMR $(500 \mathrm{MHz}$, $\left.\mathrm{CDCl}_{3}\right) \delta 16.47(\mathrm{~s}, 1 \mathrm{H}, \mathrm{H}-1), 8.42(\mathrm{dd}, 1 \mathrm{H}, J$ $=9.1,2.5 \mathrm{~Hz}), 7.80(\mathrm{~d}, 1 \mathrm{H}, J=2.5 \mathrm{~Hz}), 7.09(\mathrm{~s}$, $4 \mathrm{H}), 6.88(\mathrm{~d}, 1 \mathrm{H}, J=9.1 \mathrm{~Hz}), 4.97$ (sept, $1 \mathrm{H}$, $J=6.1 \mathrm{~Hz}), 4.20\left(\mathrm{~s}, 4 \mathrm{H}, \mathrm{H}-11,11^{\prime}\right), 2.44(2 \mathrm{~s}$, $18 \mathrm{H}, \mathrm{H}-12,13), 1.30(\mathrm{~d}, 6 \mathrm{H}, J=6.1 \mathrm{~Hz}) ;{ }^{13} \mathrm{C}$ NMR $\left(125 \mathrm{MHz} \mathrm{MHz}, \mathrm{CDCl}_{3}\right) \delta$ 291.1, 208.4, $156.4,150.3,144.7,143.1,139.3,139.1,129.5$, 124.1, 117.4, 112.7, 77.6, 51.5, 21.1, 21.0, 19.4; ${ }^{1} \mathrm{H}$ NMR (500 MHz, $\left.\mathrm{CD}_{2} \mathrm{Cl}_{2}\right) \delta 16.42$ (s, $1 \mathrm{H}$, $\mathrm{H}-1), 8.46(\mathrm{dd}, 1 \mathrm{H}, \mathrm{J}=9.1,2.5 \mathrm{~Hz}, \mathrm{H}-5), 7.80$ $(\mathrm{d}, 1 \mathrm{H}, J=2.5 \mathrm{~Hz}, \mathrm{H}-3), 7.10$ (s, 4H, H-16), $6.94(\mathrm{~d}, 1 \mathrm{H}, J=9.1 \mathrm{~Hz}, \mathrm{H}-6), 5.01$ (sept, 1H, $J=6.1 \mathrm{~Hz}, \mathrm{H}-8), 4.22$ (s, 4H, H-11,11'), 2.47 (2s, 18H, H-12,13), $1.30(\mathrm{~d}, J=6.1 \mathrm{~Hz}, 6 \mathrm{H}$, $\left.\mathrm{H} 9,9^{\prime}\right) ;{ }^{13} \mathrm{C} \mathrm{NMR}\left(125 \mathrm{MHz}, \mathrm{CD}_{2} \mathrm{Cl}_{2}\right) \delta 289.1$ (C-1), 208.2 (C-10), 156.8 (C-7), 150.3, 145.0 (C-2), 143.5 (C-4), 139.6 (C-15), 139.3, 129.8 (C-16), 124.5 (C-5), 117.2 (C-3), 113.3 (C-6), 78.2 (C-8), $52.0\left(\mathrm{C}-11,11^{\prime}\right), 21.3\left(\mathrm{C}-9,9^{\prime}\right), 21.2$ (C-12), 19.4 (C-13) (where assignments of the NMR signals (based on 2D ${ }^{1} \mathrm{H},{ }^{13} \mathrm{C}$-chemical shift correlated spectra: GHSQC, GHMBC) are given, they are unambiguous and refer to the arbitrary numbering shown on Scheme 1); MS (ESI) $m / z$ (rel intensity) 636 $[\mathrm{M}-\mathrm{Cl}]^{+}$; $\operatorname{HRMS}$ (IE): $\mathrm{m} / \mathrm{z}$ calcd for $[\mathrm{M}]^{+}$ $\left(\mathrm{C}_{31} \mathrm{H}_{37} \mathrm{~N}_{3} \mathrm{O}_{3}{ }^{35} \mathrm{Cl}_{2}{ }^{102} \mathrm{Ru}\right):$ 671.1255. found 671.1229; Anal. Calcd. for $\mathrm{C}_{31} \mathrm{H}_{37} \mathrm{Cl}_{2} \mathrm{~N}_{3} \mathrm{O}_{3} \mathrm{Ru}$ : C, 55.44; H, 5.55; N, 6.26. found: C, 55.35; H,
$5.70 ; \mathrm{N}, 6.09$.

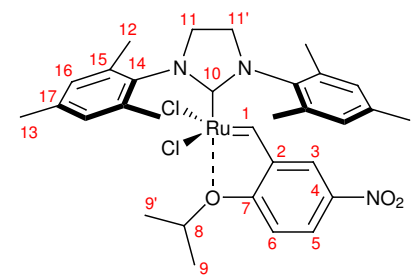

$6 b$

Scheme 1

Catalyst 7b: green microcrystalline solid (83\%). IR (KBr): $\nu$ 2922, 2853, 1740, 1666, 1607, 1520, 1484, 1448, 1423, 1401, 1378, 1337, 1262, 1200, 1158, 1114, 1097, 1034, 968, 902, $853,801,736,579,453,427 \mathrm{~cm}^{-1} ;{ }^{1} \mathrm{H}$ NMR $\left(500 \mathrm{MHz}, \mathrm{CDCl}_{3}\right) \delta 1.29(\mathrm{~d}, 6 \mathrm{H}, J=6.1 \mathrm{~Hz})$, 2.40-2.47 (m, 18H), $4.18(\mathrm{~s}, 4 \mathrm{H}), 4.94-5.02$ (heptet, $1 \mathrm{H}, J=6.1 \mathrm{~Hz}), 7.06(\mathrm{~s}, 1 \mathrm{H}), 7.08$ (bs, 4H), 7.65 (bs, 1H), 7.77 (dd, $1 \mathrm{H}, J=8.4$, $1.9 \mathrm{~Hz}), 16.73$ (s 1H); ${ }^{13} \mathrm{C}$ NMR $(125 \mathrm{MHz}$, $\left.\mathrm{CDCl}_{3}\right) \delta 21.0,51.5,76.7,108.2,119.2,121.4$, 129.4, 139.1, 145.3, 147.8, 151.5, 207.6, 288.7; MS (EI) $m / z$ (rel intensity) $671\left(5,[\mathrm{M}]^{+\cdot}\right)$, 670 (3), 442 (12), 406 (18), 405 (11), 404 (13), 403 (10), 338 (21), 337 (14), 323 (28), 305 (33), 304 (94), 303 (92), 301 (14), 289 (21), 167 (63), 166 (22), 165 (39), 161 (11), 159 (10), 158 (21), 155 (11), 153 (31), 152 (10), 148 (10), 146 (33), 145 (17), 144 (15), 139 (37), 137 (10), 136 (13), 135 (11), 131 (11), 130 (11), 123 (12), 122 (14), 121 (25), 120 (10), 119 (12), 107 (17), 93 (16), 91 (25), 79 (12), 78 (15), 77 (29), 65 (30), 63 (15), 44 (10), 43 (100), 42 (31), 41 (76), 40 (12), 39 (48), 38 (10), 36 (42); HRMS (EI): calcd. for $[\mathrm{M}]^{+\cdot}\left(\mathrm{C}_{31} \mathrm{H}_{37} \mathrm{O}_{3} \mathrm{~N}_{3}{ }^{35} \mathrm{Cl}_{2}{ }^{102} \mathrm{Ru}\right.$ : 671.12555. found: 671.12151 .

Catalyst 10: Second generation Grubbs' catalyst $\mathbf{1 b}(0.089 \mathrm{~g}, 0.10 \mathrm{mmol})$ and $\mathrm{CuCl}$ $(0.013,0.13 \mathrm{mmol})$ were placed in a Schlenk flask. Methylene chloride ( $3 \mathrm{~mL}$ ) was added under argon and then a solution of 2-isopropoxy-3-methoxy-5-nitrostyrene 16 (0.031 $\mathrm{g}, 0.13 \mathrm{mmol})$ in methylene chloride $(2 \mathrm{~mL})$ was added. The solution was stirred at room temperature for $15 \mathrm{~min}$. The solvent was evaporated and the product was purified by column chromatography ( $c$-hexane-AcOEt $4: 1)$. The product $(0.034 \mathrm{~g}, 46 \%)$ was obtained as a green microcrystalline solid. IR (film): $\nu$ 3531, 2974, 2927, 2855, 1937, 1694, 1631, 1607, 1572, 1523, 1481, 1464, 1446, 1400, $1381,1338,1267,1235,1217,1183,1163,1147$, 
$1097,1037,978,909,889,853,818,776,742$ 695, 646, 621, 579, $404 \mathrm{~cm}^{-1} ;{ }^{1} \mathrm{H}$ NMR (500 $\left.\mathrm{MHz}, \mathrm{CDCl}_{3}\right) \delta 1.35(\mathrm{~d}, 6 \mathrm{H}, J=6.1 \mathrm{~Hz})$, 2.43 (bs, 18H), 3.85 (s, 3H), $4.19(\mathrm{~s}, 4 \mathrm{H}), 5.84$ (heptet, $1 \mathrm{H}, J=6.1 \mathrm{~Hz}), 7.10(\mathrm{~s}, 4 \mathrm{H}), 7.43$ $(\mathrm{d}, 1 \mathrm{H}, J=2.5 \mathrm{~Hz}), 7.94(\mathrm{~d}, 1 \mathrm{H}, J=2.5 \mathrm{~Hz})$, $16.38(\mathrm{~s}, 1 \mathrm{H}) ;{ }^{13} \mathrm{C}$ NMR $\left(125 \mathrm{MHz}, \mathrm{CDCl}_{3}\right) \delta$ 293.8, 208.6, 153.2, 150.5, 149.1, 146.4, 145.2, $143.6,143.3,139.2,132.0,129.4,125.3,124.0$, $115.2,114.0,111.3,107.9,106.3,83.1,56.7$, 56.5, 56.2, 38.2, 29.6, 26.9, 22.4, 21.9, 21.0; MS (LSIMS) $m / z$ (rel intensity) 701.1 ([M] ${ }^{+\cdot}$ ) $666.2\left([\mathrm{M}-\mathrm{Cl}]^{+}\right)$; HRMS (LSIMS) calcd for $[\mathrm{M}-\mathrm{Cl}]^{+}\left(\mathrm{C}_{32} \mathrm{H}_{39} \mathrm{O}_{4} \mathrm{~N}_{3} \mathrm{ClRu}\right)$ : 667.1679; found: 667.1664 .

Catalyst 70: green solid (39\%). IR (KBr): $\nu$ 3461, 2924, 2853, 1934, 1678, 1608, 1576, 1523, $1483,1447,1380,1343,1267,1133,1085,1010$, $942,905,853,826,746,636,613,579 \mathrm{~cm}^{-1} ;{ }^{1} \mathrm{H}$ NMR $\left(500 \mathrm{MHz}, \mathrm{CDCl}_{3}\right) \delta 2.45(\mathrm{~s}, 18 \mathrm{H}), 3.95$ $(\mathrm{s}, 3 \mathrm{H}), 4.17(\mathrm{~s}, 4 \mathrm{H}), 6.93(\mathrm{~d}, 1 \mathrm{H}, J=9.3 \mathrm{~Hz})$, $7.10(\mathrm{~s}, 4 \mathrm{H}), 7.78(\mathrm{~d}, 1 \mathrm{H}, J=2.4 \mathrm{~Hz}), 8.43(\mathrm{dd}$, $1 \mathrm{H}, J=9.0,2.5 \mathrm{~Hz}), 16.39$ (s, $1 \mathrm{H}) ;{ }^{13} \mathrm{C} \mathrm{NMR}$ $\left(125 \mathrm{MHz}, \mathrm{CDCl}_{3}\right) \delta 285.5,207.5,157.5,143.9$, $143.5,139.2$, 138.6, 135.8, 129.7, 129.6, 124.2, 124.0, 116.6, 111.5, 59.5, 51.7, 20.9, 19.1; MS (ESI) $m / z$ (rel intensity) $675.4\left([\mathrm{M}+\mathrm{MeOH}]^{+}\right)$.

\subsection{A one-pot tree-step preparation of $6 b$ from 1a}

To a suspension of salt 24 (152 mg, 0.388 mmol, Strem) in $n$-hexane (7 $\mathrm{mL})$ a solution potassium tert-amylate, $\mathrm{CH}_{3} \mathrm{CH}_{2} \mathrm{C}\left(\mathrm{CH}_{3}\right)_{2} \mathrm{OK}$, $(0.22 \mathrm{~mL}, 0.372 \mathrm{mmol}$, 1.7 $\mathrm{M}$ in toluene, Fluka) was added under argon and the resulted slightly turbid, yellow solution was stirred at room temperature for 30 min. Grubbs' catalyst 1a $(255 \mathrm{mg}, 0.310$ mmol) was then added to the flask as a solid and the reaction mixture was heated to reflux for $30 \mathrm{~min}$. To the resulted brown-pink suspension a solution of $14(83.5 \mathrm{mg}, 0.403$ $\mathrm{mmol})$ in $\mathrm{CH}_{2} \mathrm{Cl}_{2}(7 \mathrm{~mL})$ and solid $\mathrm{CuCl}$ (33.8 $\mathrm{mg}, 0.341 \mathrm{mmol})$ were added at RT. After 1 $\mathrm{h}$ at $40{ }^{\circ} \mathrm{C}$ the resulted product was purified as described above to afford catalyst $\mathbf{6 b}$ as green crystals (149 mg, 72\%). Analytical data were identical with these reported above.

\section{General procedures for metathesis reactions}

\subsection{Cross-metathesis}

To a mixture of an alkene $(1.0 \mathrm{mmol})$ and a cross-metathesis partner $(2.0-4.0 \mathrm{mmol})$ in $\mathrm{CH}_{2} \mathrm{Cl}_{2}(5 \mathrm{~mL})$ was added a solution of a Ru-catalyst (0.01-0.05 mmol, 1-5 mol\%) in $\mathrm{CH}_{2} \mathrm{Cl}_{2}$ (1 mL). The resulting mixture was stirred at $25-45{ }^{\circ} \mathrm{C}$ for $0.5-16 \mathrm{~h}$. The solvent was removed under reduced pressure. The crude product was purified by flash chromatography (c-hexane-ethyl acetate).

\subsection{RCM and enyne metathesis}

To a mixture of an alkene $(1.0 \mathrm{mmol})$ in $\mathrm{CH}_{2} \mathrm{Cl}_{2}(50 \mathrm{~mL}, c=0.02 \mathrm{M})$ was added a solution of a Ru-catalyst (0.01-0.05 mmol, 1-5 $\mathrm{mol} \%$ ) in $\mathrm{CH}_{2} \mathrm{Cl}_{2}(1 \mathrm{~mL})$. The resulting mixture was stirred at $0-45{ }^{\circ} \mathrm{C}$ for $0.5-16 \mathrm{~h}$. The solvent was removed under reduced pressure. The crude product was purified by flash chromatography (c-hexane-ethyl acetate).

(Z)-5-Methoxy-1-(methoxymethyl)-3-[(4-methylphenyl)sulfonyl]-1 $H$-indol-4-yl-1-propenyl phenyl sulfone (30): ${ }^{9}$ Colourless crystals, $\mathrm{mp}=140-141{ }^{\circ} \mathrm{C}(54 \%)$. IR (KBr): $\nu$ 3532, 3119, 2926, 2852, 1627, 1514, 1286, 1143, 1085, 1027, 798, 676, $593 \mathrm{~cm}^{-1}$; ${ }^{1} \mathrm{H} \mathrm{NMR}\left(500 \mathrm{MHz}, \mathrm{CDCl}_{3}\right) \delta 3.32(\mathrm{~s}, 3 \mathrm{H})$, $3.58(\mathrm{~s}, 3 \mathrm{H}), 3.96(\mathrm{dd}, 2 \mathrm{H}, J=5.8,1.5 \mathrm{~Hz})$, $5.46(\mathrm{~s}, 2 \mathrm{H}), 5.74(\mathrm{dt}, 1 \mathrm{H}, J=15.0,1.5 \mathrm{~Hz})$, $6.54(\mathrm{dt}, 1 \mathrm{H}, J=15.0,5.8 \mathrm{~Hz}), 6.92(\mathrm{~d}, 1 \mathrm{H}$, $J=9.0 \mathrm{~Hz}), 7.25-7.32(\mathrm{~m}, 2 \mathrm{H}), 7.39-7.56(\mathrm{~m}$, $4 \mathrm{H}), 7.66-7.77\left(\mathrm{AA}^{\prime} \mathrm{XX}^{\prime}, 4 \mathrm{H}\right), 8.08(\mathrm{~s}, 1 \mathrm{H}) ;{ }^{13} \mathrm{C}$ NMR $\left(125 \mathrm{MHz}, \mathrm{CDCl}_{3}\right) \delta$ 21.6, 29.7, 56.2, 56.4, 78.6, 109.6, 110.6, 115.4, 116.6, 124.4, $126.5,127.2,128.9,129.1,130.1,132.7,137.4$, 139.5, 141.3, 144.3, 145.8, 154.2; MS (LSIMS) $\mathrm{m} / \mathrm{z}$ (rel intensity) $548[\mathrm{M}+\mathrm{Na}]^{+}$; MS (EI) $\mathrm{m} / \mathrm{z}$ (rel intensity) $525\left(5,[\mathrm{M}]^{+\cdot}\right), 494(6), 384$ (52), 370 (37), 366 (10), 349 (12), 306 (16), 229 (47), 214 (20), 198 (31), 184 (16), 169 (17), 105 (38), 77 (19); Anal. Calcd. for $\mathrm{C}_{17} \mathrm{H}_{27} \mathrm{NO}_{6} \mathrm{~S}_{2}$ : C, 61.70; H, 5.18; N, 2.66. found: C, 61.46; H, $5.35 ; \mathrm{N}, 2.76$.

Methyl-7-[1-(tert-butyl)-1,1-dimethylsilyl]oxy-2-heptenoate (31): ${ }^{12}$ Colorless oil, $(E):(Z)=95: 5$ (95\%). IR (film): $\nu$ 2952, 2933, $2859,1729,1659,1472,1437,1389,1317,1258$,

\footnotetext{
12 Nicolaou, K. C.; Hwang, C.-K.; Marron, B. E.; DeFrees, S. A.; Couladouros, E. A. J. Am. Chem. Soc. 1990, 112, $3040-3054$.
} 
$1201,1165,1102,1040,983,838,777 \mathrm{~cm}^{-1} ;{ }^{1} \mathrm{H}$ NMR $\left(500 \mathrm{MHz}, \mathrm{CDCl}_{3}\right) \delta \quad 0.03(\mathrm{~s}, 6 \mathrm{H}), 0.88$ $(\mathrm{s}, 9 \mathrm{H}), 1.46-1.57(\mathrm{~m}, 4 \mathrm{H}), 2.17-2.25(\mathrm{~m}, 2 \mathrm{H})$, $3.61(\mathrm{t}, 2 \mathrm{H}, J=5.9 \mathrm{~Hz}), 3.71(\mathrm{~s}, 3 \mathrm{H}), 5.81(\mathrm{dt}$, $1 \mathrm{H}, J=15.7,1.6 \mathrm{~Hz}), 6.96(\mathrm{dt}, 1 \mathrm{H}, J=15.7$, $7.0 \mathrm{~Hz}) ;{ }^{13} \mathrm{C}$ NMR $\left(125 \mathrm{MHz}, \mathrm{CDCl}_{3}\right) \delta 167.1$, 149.4, 121.0, 62.7, 51.3, 32.2, 31.9, 25.9, 24.4, 18.3, -5.3; MS (EI) $\mathrm{m} / \mathrm{z}$ (rel intensity) 257 (1, $\left.[\mathrm{M}-15]^{+}\right), 241$ (3), $217(4), 215$ (23), 183 (15), 171 (0.5), 155 (1), 139 (3), 119 (3), 101 (3), 89 (100), 81 (53), 79 (13), 75 (32), 73 (25), 59 (24), 47 (11), 41 (21), 39 (10); HRMS (ESI) calcd for [M+Na] ${ }^{+}\left(\mathrm{C}_{14} \mathrm{H}_{28} \mathrm{O}_{3} \mathrm{SiNa}\right): 295.1700$. found: 295.1691.

7-[1-(tert-butyl)-1,1-dimethylsilyl]oxy-2-heptenenitrile (32): ${ }^{13}$ Yellow oil, $(Z):(E)=$ 2.7:1 (90\%). IR (film): $\nu$ 2953, 2931, 2858, 2222, 1633, 1523, 1472, 1388, 1344, 1255, 1179, $1101,1006,978,836,776 \mathrm{~cm}^{-1} ;{ }^{1} \mathrm{H}$ NMR (500 $\left.\mathrm{MHz}, \mathrm{CDCl}_{3}\right) \delta 0.07(\mathrm{~s}, 6 \mathrm{H}), 0.89(\mathrm{~s}, 9 \mathrm{H})$, $1.49-1.54$ and $1.54-1.58(2 \mathrm{~m}, 4 \mathrm{H}), 2.42-2.48$ $(\mathrm{m}, 2 \mathrm{H}), 3.60-3.64(\mathrm{~m}, 2 \mathrm{H}), 5.29-5.35(\mathrm{~m}$, $1 \mathrm{H}), 6.45-6.51(\mathrm{~m}, 1 \mathrm{H}) ;{ }^{13} \mathrm{C}$ NMR $(125 \mathrm{MHz}$, $\left.\mathrm{CDCl}_{3}\right) \delta 155.0,118.2,99.6,62.5,32.0,31.6$, $25.9,24.6,21.9,-5.3$; MS (EI) $\mathrm{m} / z$ (rel intensity) $238(0.5), 224$ (3), 183 (15), 182 (100), 166 (1), 152 (9), 138 (1), 126 (22), 108 (10), 101 (6), 81 (7), 75 (74), 73 (21), 59 (22), 57 (11), 49 (17), 47 (19), 41 (25), 39 (16) (GC/MS for (Z)-isomer); MS (EI) $\mathrm{m} / \mathrm{z}$ (rel intensity) 238 (0.5), 224 (3), 183 (14), 182 (73), 166 (1), 155 (9), 140 (2), 126 (15), 115 (7), 108 (8), 101 (11), 81 (15), 75 (100), 73 (26), 59 (25), 57 (11), 56 (11), 47 (17), 45 (18), 41 (24), 39 (16) (GC/MS for (E)-isomer); HRMS (ESI) calcd for [M+Na] ${ }^{+}$ ( $\left.\mathrm{C}_{13} \mathrm{H}_{25} \mathrm{NOSiNa}\right): 262.1598$. found: 262.1610.

Diethyl 2-[3-cyano-2-propenyl]malonate (33): ${ }^{14}$ Colorless oil, $(E):(Z)=1: 2(87 \%)$. IR (film): $\nu$ 2986, 2941, 2223, 1749, 1732, 1467, 1447, 1371, 1339, 1228, 1177, 1097, 1032, $859 \mathrm{~cm}^{-1}$; (E)-isomer: ${ }^{1} \mathrm{H}$ NMR $(500 \mathrm{MHz}$, $\left.\mathrm{CDCl}_{3}\right) \delta 1.28(\mathrm{t}, 6 \mathrm{H}, J=3.6 \mathrm{~Hz}), 2.77-2.81$ $(\mathrm{m}, 2 \mathrm{H}), 3.46(\mathrm{t}, 1 \mathrm{H}, J=3.6 \mathrm{~Hz}), 4.19-4.25$ $(\mathrm{m}, 4 \mathrm{H}), 5.42-5.44(\mathrm{~m}, 1 \mathrm{H}), 6.66-6.78(\mathrm{~m}, 1 \mathrm{H})$; ${ }^{13} \mathrm{C}$ NMR $\left(125 \mathrm{MHz}, \mathrm{CDCl}_{3}\right) \delta 167.9,150.7$, 116.8, 102.6, 61.8, 50.2, 32.0, 14.0; (Z)-isomer: ${ }^{1} \mathrm{H}$ NMR $\left(500 \mathrm{MHz}, \mathrm{CDCl}_{3}\right) \delta 1.28(\mathrm{t}, 6 \mathrm{H}$, $J=3.6 \mathrm{~Hz}), 2.97-3.00(\mathrm{~m}, 2 \mathrm{H}), 3.51(\mathrm{t}, 1 \mathrm{H}$, $J=3.6 \mathrm{~Hz}), 4.19-4.25(\mathrm{~m}, 4 \mathrm{H}), 5.42-5.44(\mathrm{~m}$, $1 \mathrm{H}), 6.51-6.60(\mathrm{~m}, 1 \mathrm{H}) ;{ }^{13} \mathrm{C}$ NMR $(125 \mathrm{MHz}$, $\left.\mathrm{CDCl}_{3}\right) \delta 167.8,149.8,115.2,102.1,61.8,50.3$, 30.4, 14.0; MS (EI) $m / z$ (rel intensity) 225 (7),
197 (12), 180 (38), 179 (27), 169 (12), 152 (24), 151 (68), 134 (20), 124 (21), 123 (82), 107 (55), 106 (63), 93 (24), 80 (100), 79 (80), 78 (25), 69 (18), 55 (24), 53 (48), 52 (52), 39 (25).

Phenyl [(E)-3-phenyl-1-propenyl] sulfone (34): ${ }^{9}$ Colourless oil (84\%). IR (film): $\nu$ 3057, 2921, 1630, 1496, 1384, 1315, 1127, 976, $792 \mathrm{~cm}^{-1} ;{ }^{1} \mathrm{H}$ NMR $\left(400 \mathrm{MHz}, \mathrm{CDCl}_{3}\right)$ $\delta 3.59(\mathrm{dd}, 2 \mathrm{H}, J=6.4,1.7 \mathrm{~Hz}), 6.05(\mathrm{~d}$, $1 \mathrm{H}, J=9.9 \mathrm{~Hz}), 6.16(\mathrm{dt}, 1 \mathrm{H}, J=15.1,1.7$ $\mathrm{Hz}), 6.37(\mathrm{~d}, 1 \mathrm{H}, J=16.5 \mathrm{~Hz}), 6.56(\mathrm{dd}, 1 \mathrm{H}$, $J=16.5,9.9 \mathrm{~Hz}), 7.11(\mathrm{dt}, 1 \mathrm{H}, J=15.1,6.4$ $\mathrm{Hz}), 7.14-7.18,7.24-7.37(2 \mathrm{~m}, 5 \mathrm{H}) ;{ }^{13} \mathrm{C}$ NMR $\left(100 \mathrm{MHz}, \mathrm{CDCl}_{3}\right) \delta 37.7,127.1,128.7,128.8$, $128.8,128.9,129.7,136.0,137.4,147.7$; MS (EI) $\mathrm{m} / \mathrm{z}$ (rel intensity) $208\left(11,[\mathrm{M}]^{+\cdot}\right), 163(3)$, 142 (5), 128 (2), 117 (100), 103 (2), 91 (34), 89 (7), 77 (6), 65 (13); HRMS (EI) calcd for [M] ${ }^{+\cdot}$ $\left(\mathrm{C}_{11} \mathrm{H}_{12} \mathrm{O}_{2} \mathrm{~S}\right):$ 208.0558. found 208.0556.

(E)-6-[1-(tert-butyl)-1,1-dimethylsilyl]-
oxy-1-hexenyl(diphenyl)phosphine oxide (35): ${ }^{15}$ Colourless oil (82\%). IR (KBr): $\nu$ 3059, 2948, 2959, 1617, 1438, 1252, 1189, 1097, 839, $701,528 \mathrm{~cm}^{-1} ;{ }^{31} \mathrm{P} \mathrm{NMR}\left(\mathrm{CDCl}_{3}, 202 \mathrm{MHz}\right): \delta$ $24.8 ;{ }^{1} \mathrm{H} \mathrm{NMR}\left(\mathrm{CDCl}_{3}, 500 \mathrm{MHz}\right): \delta 7.71-7.64$ $(\mathrm{m}, 4 \mathrm{H}), 7.52-7.40(\mathrm{~m}, 6 \mathrm{H}), 6.71(\mathrm{ddt}, 1 \mathrm{H}, J$ $=19.5,17.0,6.5 \mathrm{~Hz}), 6.22(\mathrm{ddt}, 1 \mathrm{H}, J=24.5$, 17.0, $1.5 \mathrm{~Hz}), 3.61-3.57(\mathrm{~m}, 2 \mathrm{H}), 2.34-2.2 .26$ $(\mathrm{m}, 2 \mathrm{H}), 1.57-1.49(\mathrm{~m}, 4 \mathrm{H}), 0.86(\mathrm{~s}, 9 \mathrm{H}), 0.01$ $(\mathrm{s}, 6 \mathrm{H}) ;{ }^{13} \mathrm{C} \mathrm{NMR}\left(\mathrm{CDCl}_{3}, 126 \mathrm{MHz}\right): \delta 152.5$ $(\mathrm{d}, J=1.9 \mathrm{~Hz}), 133.0(\mathrm{~d}, J=105.0 \mathrm{~Hz}), 131.5$ $(\mathrm{d}, J=2.6 \mathrm{~Hz}), 131.2(\mathrm{~d}, J=9.8 \mathrm{~Hz}), 128.4(\mathrm{~d}$, $J=12.1 \mathrm{~Hz}), 122.3(\mathrm{~d}, J=103.3), 62.7(\mathrm{~s}), 34.1$ (d, $J=16.9 \mathrm{~Hz}), 32.28$ (s), $25.88(\mathrm{~s}), 24.26(\mathrm{~s})$, 18.25 (s), -5.37 (s); MS (EI) $m / z$ (rel intensity) $414\left(1,[\mathrm{M}]^{+\cdot}\right), 399$ (3), 357 (100), 202 (10), 135 (5), 115 (3), 81 (4), 75 (8), 59 (4); HRMS (EI) calcd for [M] $]^{+\cdot}\left(\mathrm{C}_{24} \mathrm{H}_{35} \mathrm{SiO}_{2} \mathrm{P}\right): 414.2144$. found 414.2162; Anal. calcd for $\mathrm{C}_{24} \mathrm{H}_{35} \mathrm{SiO}_{2} \mathrm{P}$ : C, 69.53; H, 8.51. found: C, 68.99; H, 8.79.

\section{4-(2-Methyl-4-nitro-1H-indol-3-yl)-2-}

-butenenitrile (36): Orange crystals, $(E):(Z)$ $=1: 2.4(69 \%)$. IR (KBr): $\nu$ 3313, 3056, 2928, 2218, 1609, 1568, 1512, 1432, 1338, 1304, 1253, 1191, 1116, 993, 787, $724 \mathrm{~cm}^{-1}$; (Z)-isomer: ${ }^{1} \mathrm{H}$ NMR $\left(500 \mathrm{MHz}, \mathrm{CDCl}_{3}\right) \delta 2.47(\mathrm{~s}, 3 \mathrm{H})$, $3.88(\mathrm{dd}, 2 \mathrm{H}, J=6.9,1.6 \mathrm{~Hz}), 5.41(\mathrm{dt}, 1 \mathrm{H}$, $J=10.9,1.6 \mathrm{~Hz}), 6.61-6.68(\mathrm{~m}, 1 \mathrm{H}), 7.13(\mathrm{t}$, $1 \mathrm{H}, J=7.9 \mathrm{~Hz}), 7.61(\mathrm{dd}, 1 \mathrm{H}, J=7.9,1.0$ $\mathrm{Hz}), 7.77(\mathrm{dd}, 1 \mathrm{H}, J=7.9,1.0 \mathrm{~Hz}) ;{ }^{13} \mathrm{C}$ NMR $\left(125 \mathrm{MHz}, \mathrm{CDCl}_{3}\right) \delta 156.1,155.2,153.2,141.3$,

\footnotetext{
${ }^{13}$ Crowe, William E.; Goldberg, Daniel R. J. Am. Chem. Soc. 1995, 117, 5162 - 5163.

14 Randl, S.; Gessler, S.; Wakamatsu, H.; Blechert, S. Synlett 2001, 430-432.

15 Demchuk, O. M.; Pietrusiewicz, K. M.; Michrowska, A.; Grela, K. Org. Lett. 2003, 5, 3217-3220.
} 
138.8, 138.0, 119.1, 117.5, 116.9, 104.4, 98.6, 29.4, 11.3; MS (EI) $\mathrm{m} / \mathrm{z}$ (rel intensity) 241 (58), 225 (12), 224 (78), 207 (11), 194 (31), 193 (100), 192 (19), $181(12), 180(13), 179$ (73), 169 (23), 168 (21), 167 (12), 152 (12), 127 (7), 115 (4), 77 (3); HRMS (EI) calcd for [M] ${ }^{+}$. $\left(\mathrm{C}_{13} \mathrm{H}_{11} \mathrm{~N}_{3} \mathrm{O}_{2}\right)$ : 241.0851. found: 241.0854 .

8-[1-(tert-Butyl)-1,1-dimethylsilyl]oxy-3-octen-2-one (37): ${ }^{16}$ Yellow oil, $(E):(Z)=99: 1$ $(95 \%) .{ }^{1} \mathrm{H}$ NMR $\left(500 \mathrm{MHz}, \mathrm{CDCl}_{3}\right) \delta 0.04$ $(\mathrm{s}, 6 \mathrm{H}), 0.88(\mathrm{~s}, 9 \mathrm{H}), 1.50-1.56(\mathrm{~m}, 4 \mathrm{H}), 2.22$ (s, 3H), 2.21-2.27 (m, 2H), 3.58-2.63 (m, 2H), $6.07(\mathrm{dt}, 1 \mathrm{H}, J=15.9,1.4 \mathrm{~Hz}), 6.79(\mathrm{dt}, 1 \mathrm{H}, J=$ $15.9,6.9 \mathrm{~Hz}) ;{ }^{13} \mathrm{C}$ NMR $\left(125 \mathrm{MHz}, \mathrm{CDCl}_{3}\right) \delta$ 198.6, 148.2, 131.4, 62.7, 32.2, 32.1, 26.8, 25.9, 24.5, 18.3, -5.3; MS (EI) $m / z$ (rel intensity) 256 (0.1), 241 (2), 200 (15), 199 (96), 157 (10), 156 (20), 155 (100), 131 (7), 115 (5), 101 (5), 75 (36), 73 (5), 59 (3), 43 (6), 39 (2); HRMS (ESI) $[\mathrm{M}+\mathrm{Na}]^{+}\left(\mathrm{C}_{14} \mathrm{H}_{28} \mathrm{O}_{2} \mathrm{SiNa}\right)$ : calcd. 279.1751. found: 279.1749 .

7-[1-(tert-butyl)-1,1-dimethylsilyl]oxy-2-methyl-2-heptenenitrile (38): ${ }^{16}$ Colorless oil, $(E):(Z)=1: 2(58 \%) .{ }^{1} \mathrm{H}$ NMR $(500 \mathrm{MHz}$, $\left.\mathrm{CDCl}_{3}\right) \delta 0.08(\mathrm{~s}, 6 \mathrm{H}), 0.93(\mathrm{~s}, 9 \mathrm{H}), 1.25-1.46$ (m, 2H), 1.48-1.64 (m, 2H), 1.87-2.08 (m, 3H), 2.18-2.42 (m, 2H), 3.60-3.69 (m, 2H), $6.16(\mathrm{t}$, $1 \mathrm{H}, J=7.6 \mathrm{~Hz}) \mathrm{ppm} ;{ }^{13} \mathrm{C}$ NMR $(125 \mathrm{MHz}$, $\left.\mathrm{CDCl}_{3}\right) \delta$ 148.3, 118.1, 109.3, 62.6, 32.2, 31.3, 25.9, 24.9, 24.7, 21.9, -5.3; (Z)-isomer: MS (EI) $\mathrm{m} / z$ (rel intensity) $252\left(0.5,[\mathrm{M}-\mathrm{H}]^{+}\right) 238(2)$, 197 (13), 196 (100), 166 (3), 140 (9), 128 (3), 122 (3), 101 (3), 75 (56), 73 (20), 59 (12), 51 (7), 45 (11), 41 (19), 39 (10); (E)-somer: MS (EI) $m / z$ (rel intensity) $253\left(1,[\mathrm{M}]^{+\cdot}\right), 238(3), 197$ (12), 196 (100), 169 (1), 140 (7), 115 (7), 101 (8), 75 (91), 73 (18), 59 (12), 51 (7), 45 (11), 41 (19), 39 (10); HRMS (ESI) calcd for [M+Na] ${ }^{+}$ $\left(\mathrm{C}_{14} \mathrm{H}_{27} \mathrm{NOSiNa}\right):$ 276.1754. found: 276.1773.

(E)-6-[1-(tert-butyl)-1,1-dimethylsilyl]oxy-1-hexenyl phenyl sulfone (39): ${ }^{9}$ Colourless oil (90\%). IR (film): $\nu$ 2952, 2931, 2858, 1321, 1148, 1088, 836, 777, 753, 688, 596, 553 $\mathrm{cm}^{-1} ;{ }^{1} \mathrm{H}$ NMR $\left(200 \mathrm{MHz}, \mathrm{CDCl}_{3}\right) \delta 0.03$ (s, $6 \mathrm{H}), 0.88(\mathrm{~s}, 9 \mathrm{H}), 1.46-1.60(\mathrm{~m}, 4 \mathrm{H}), 2.20-2.35$ $(\mathrm{m}, 2 \mathrm{H}), 3.53-3.62(\mathrm{~m}, 2 \mathrm{H}), 6.32(\mathrm{dt}, 1 \mathrm{H}, J$ $=15.1,1.5 \mathrm{~Hz}), 7.00(\mathrm{dt}, 1 \mathrm{H}, J=15.1,6.8$ $\mathrm{Hz}), 7.47-7.93(\mathrm{~m}, 5 \mathrm{H}) ;{ }^{13} \mathrm{C}$ NMR $(50 \mathrm{MHz}$, $\left.\mathrm{CDCl}_{3}\right) \delta-5.37,18.26,24.04,25.90,31.22$, $32.00,62.50,127.50,127.65,129.18,130.43$, 133.16, 140.72, 147.00; MS (EI) $m / z$ (rel intensity) 299 (16), 298 (22), 297 (100), 199 (10), 135 (50), 125 (5), 81 (5), 79 (5), 77 (4), 75 (13),
73 (8); HRMS (LSIMS): calcd for $[\mathrm{M}+\mathrm{H}]^{+}$ $\left(\mathrm{C}_{18} \mathrm{H}_{31} \mathrm{O}_{3} \mathrm{SSi}\right)$ : 355.1763. found 335.1768.

(E)-11-(Phenylsulfonyl)-10-undecen-1-ol (40): ${ }^{9}$ Colourless oil (81\%). IR (film): $\nu$ 3370, 2928, 2855, 1447, 1318, 1306, 1289, 1147, 1086, $753,688,595 \mathrm{~cm} \mathrm{~cm}^{-1}$; ${ }^{1} \mathrm{H}$ NMR $(500 \mathrm{MHz}$, $\left.\mathrm{CDCl}_{3}\right) \delta$ 1.24-1.35, 1.41-1.49, 1.51-1.58 (3m, $15 \mathrm{H}), 2.23(\mathrm{~m}, 2 \mathrm{H}), 3.62(\mathrm{t}, 2 \mathrm{H}, \mathrm{J}=6.6 \mathrm{~Hz})$, $6.32(\mathrm{dt}, 1 \mathrm{H}, J=15.1,1.5 \mathrm{~Hz}), 6.98(\mathrm{dt}, 1 \mathrm{H}, J$ $=15.1,6.9 \mathrm{~Hz}), 7.51-7.92(\mathrm{~m}, 5 \mathrm{H}) ;{ }^{13} \mathrm{C} \mathrm{NMR}$ $\left(125 \mathrm{MHz} \mathrm{CDCl}_{3}\right) \delta 25.6,27.4,28.8,29.0,29.2$, 29.2, 31.3, 32.6, 62.8, 127.4, 129.1, 130.2, 133.1, 140.7, 147.2; MS (ESI) m/z (rel intensity) 333 $\left([\mathrm{M}+\mathrm{Na}]^{+}\right)$; HRMS (ESI) calcd for $[\mathrm{M}+\mathrm{Na}]^{+}$ $\left(\mathrm{C}_{17} \mathrm{H}_{26} \mathrm{O}_{3} \mathrm{SNa}\right)$ : 333.1495. found 333.1515; Anal. Calcd for $\mathrm{C}_{17} \mathrm{H}_{22} \mathrm{NO}_{3} \mathrm{~S}$ : C, 65.77; $\mathrm{H}$, 8.44; S, 10.33. found: C, 65.35; H, 8.20; S, 10.27 .

$\left(S_{\mathrm{P}}\right)-(-)-[(1 E)-6$-bromohex-1-enyl](methyl)phenylphosphine oxide (41): ${ }^{15}$ Pale brown oil $(86 \%)$. $[\alpha]_{\mathrm{D}}^{20}-28.9 \mathrm{deg}\left(c \mathrm{1.32}, \mathrm{CH}_{2} \mathrm{Cl}_{2}\right)$; IR (film): $\nu$ 2936, 1629, 1437, 1294, 1181, 1115, 980, 896, 742, 696, $502 \mathrm{~cm}^{-1} ;{ }^{31} \mathrm{P}$ NMR (202 $\left.\mathrm{MHz}, \mathrm{CDCl}_{3}\right): \delta 27.4 ;{ }^{1} \mathrm{H}$ NMR $(500 \mathrm{MHz}$, $\left.\mathrm{CDCl}_{3}\right): \delta 7.81-7.69(\mathrm{~m}, 2 \mathrm{H}), 7.54-7.44(\mathrm{~m}, 3$ H), $6.66(\mathrm{ddt}, 1 \mathrm{H}, J=19.4,17.0,6.5 \mathrm{~Hz}), 6.02$ $(\mathrm{ddt}, 1 \mathrm{H}, J=24.9,17.0,1.6 \mathrm{~Hz}), 3.39(\mathrm{t}, 2 \mathrm{H}, J$ $=6.7), 2.31-2.24(\mathrm{~m}, 2 \mathrm{H}), 1.92-1.83(\mathrm{~m}, 2 \mathrm{H})$, $1.75(\mathrm{~d}, 3 \mathrm{H}, \mathrm{J}=13.2 \mathrm{~Hz}), 1.65-1.58(\mathrm{~m}, 2 \mathrm{H})$; ${ }^{13} \mathrm{C} \mathrm{NMR}\left(126 \mathrm{MHz}, \mathrm{CDCl}_{3}\right): \delta 149.8(\mathrm{~d}, \mathrm{~J}=$ $1.7 \mathrm{~Hz}), 134(\mathrm{~d}, J=90.6 \mathrm{~Hz}), 131.6(\mathrm{~d}, J=$ $2.7 \mathrm{~Hz}), 130.0(\mathrm{~d}, 9.7 \mathrm{~Hz}), 128.6(\mathrm{~d}, J=11.8$ $\mathrm{Hz}), 124.0(\mathrm{~d}, J=99.6 \mathrm{~Hz}), 33.3(\mathrm{~d}, \mathrm{~J}=16.8$ $\mathrm{Hz}), 33.2$ (s), 32.0 (s), 26.4 (d, J = 1.1 Hz), 17.0 $(\mathrm{d}, J=74.5 \mathrm{~Hz}) ; \mathrm{MS}$ (ESI) $\mathrm{m} / \mathrm{z}$ (rel intensity) $301(12)[\mathrm{M}+\mathrm{H}]^{+} ; 323(100)[\mathrm{M}+\mathrm{Na}]^{+}$; HRMS (ESI) calcd for $[\mathrm{M}+\mathrm{Na}]^{+}\left(\mathrm{C}_{13} \mathrm{H}_{18} \mathrm{BrOPNa}\right)$ : 323.0171. found 323.0168.

Methyl (E)-4-(2-methyl-6-nitro-1 $H$-indol-3-yl)-2-butenoate (42): Yellow crystalline solid (91\%). IR (KBr): $\nu$ 3364, 2953, 2904, 1707, 1655, 1504, 1324, 1215, $750 \mathrm{~cm}^{-1} ;{ }^{1} \mathrm{H}$ NMR (500 MHz, $\left.\mathrm{CDCl}_{3}\right): \delta 2.42(\mathrm{~s}, 3 \mathrm{H}), 3.61$ $(\mathrm{dd}, 2 \mathrm{H}, J=1.7,6.0 \mathrm{~Hz}), 3.70(\mathrm{~s}, 3 \mathrm{H}), 5.74$ $(\mathrm{dt}, 1 \mathrm{H}, J=1.7,15.7 \mathrm{~Hz}), 7.09(\mathrm{dt}, 1 \mathrm{H}, J$ $=6.0,15.7 \mathrm{~Hz}), 7.42(\mathrm{~d}, 1 \mathrm{H}, J=8.8 \mathrm{~Hz})$, $7.98(\mathrm{dd}, 1 \mathrm{H}, J=2.0,8.8 \mathrm{~Hz}), 8.24(\mathrm{~d}, 1 \mathrm{H}$, $J=2.0 \mathrm{~Hz}), 8.51$ (br. s, $1 \mathrm{H}) ;{ }^{13} \mathrm{C} \mathrm{NMR}(125$ $\left.\mathrm{MHz} \mathrm{CDCl}_{3}\right)$ : 12.0, 26.7, 51.5, 107.2, 108.8, $115.4,117.5,121.4,133.2,133.6,138.9,142.6$, 146.7, 167.0; MS (EI) $m / z$ (rel intensity) 274 $\left(100,[\mathrm{M}]^{+\cdot}\right), 259$ (75), 242 (63), 215 (38), 199 (11), 189 (15), 175 (15), 168 (53), 154 (18),

\footnotetext{
16 Grela, K.; Harutyunyan; S., Michrowska, A. Angew. Chem. Int. Ed. 2002, 41, $4038-4040$.
} 
143 (31), 127 (12), 115 (12), 84 (17); HRMS (EI) calcd for $[\mathrm{M}]^{+\cdot}\left(\mathrm{C}_{14} \mathrm{H}_{14} \mathrm{O}_{4} \mathrm{~N}_{2}\right): 247.0954$. found 274.0959; Anal. calcd for $\mathrm{C}_{14} \mathrm{H}_{14} \mathrm{O}_{4} \mathrm{~N}_{2}$ : C, 61.31; H, 5.14; N, 10.21. found: C, 61.05; H, 5.22; N, 10.09 .

4-(2-methyl-6-nitro-1 $H$-indol-3-yl)-2-butenenitrile (43): Yellow crystals, $(E):(Z)$ $=1: 2.4$ (76\%). IR (KBr): $\nu$ 3306, 2229, 1609, 1592, 1556, 1505, 1471, 1391, 1324, 1114, 1067, 940, 871, $792 \mathrm{~cm}^{-1} ;{ }^{1} \mathrm{H}$ NMR $(500 \mathrm{MHz}$, $\left.\mathrm{CDCl}_{3}\right) \delta 2.49(\mathrm{~s}, 3 \mathrm{H}), 3.62(\mathrm{dd}, 2 \mathrm{H}, J=$ $5.5,1.6 \mathrm{~Hz}), 5.18(\mathrm{dt}, 1 \mathrm{H}, J=16.2,1.6 \mathrm{~Hz})$, 6.50-6.58 (m, 1H), $7.15(\mathrm{t}, 1 \mathrm{H}, J=7.9 \mathrm{~Hz})$, $7.55(\mathrm{~d}, 1 \mathrm{H}, \mathrm{J}=7.9 \mathrm{~Hz}), 7.86(\mathrm{~d}, 1 \mathrm{H}, J=7.9$ $\mathrm{Hz}) ;{ }^{13} \mathrm{C}$ NMR $\left(125 \mathrm{MHz}, \mathrm{CDCl}_{3}\right) \delta$ 153.1, 152.1, 142.7, 139.1, 133.6, 132.9, 117.2, 115.9, 115.6, 107.4, 99.5, 26.7, 12.1; MS (EI) $\mathrm{m} / \mathrm{z}$ (rel intensity) $241\left(100,[\mathrm{M}]^{+\cdot}\right), 226(17), 224(27)$, 211 (11), 194 (38), 193 (18), 192 (7), 189 (45), 180 (16), 179 (19), 169 (6), 168 (12), 167 (13), 155 (13), 154 (12), 143 (32), 142 (7), 127 (9), 115 (9), 77 (6); HRMS (EI) calcd for [M] ${ }^{+}$. $\left(\mathrm{C}_{13} \mathrm{H}_{11} \mathrm{~N}_{3} \mathrm{O}_{2}\right)$ : 241.0851. found: 241.0853.

( $E$ )-3-(2-methyl-6-nitro-1 $H$-indol-3-yl)-1-propenyl(diphenyl)phosphine oxide (44): ${ }^{15}$ Yellow crystals, $\mathrm{mp}=193-194{ }^{\circ} \mathrm{C}$ (76\%). IR (KBr): $\nu$ 3368, 2961, 2854, 1587, 1505, 1468, 1261, 1104, 1065, 820, $732 \mathrm{~cm}^{-1}$; ${ }^{31} \mathrm{P}$ NMR (202 MHz, CD $\left.3(\mathrm{O}) \mathrm{CD}_{3}\right): \delta 21.4$ ppm; ${ }^{1} \mathrm{H}$ NMR $\left(500 \mathrm{MHz}, \mathrm{CD}_{3} \mathrm{~S}(\mathrm{O}) \mathrm{CD}_{3}\right): \delta$ $11.70(\mathrm{~s}, 1 \mathrm{H}), 8.16(\mathrm{~d}, 1 \mathrm{H}, J=2.0 \mathrm{~Hz}), 7.80$ $(\mathrm{dd}, 1 \mathrm{H}, J=2.1,8.8 \mathrm{~Hz}), 7.64-7.45(\mathrm{~m}, 11 \mathrm{H})$, 6.74-6.51 (m, 2H), 3.77-3.70 (m, 2H), 3.29 (s, $3 \mathrm{H}) ;{ }^{13} \mathrm{C}$ NMR $\left(125 \mathrm{MHz}, \mathrm{CD}_{3} \mathrm{~S}(\mathrm{O}) \mathrm{CD}_{3}\right): \delta$ 149.3 (s), 140.9 (d, $J=18.3 \mathrm{~Hz}), 134.0$ (d, J = $112.1 \mathrm{~Hz}), 133.5$ (s), 132.8 (s), 132.5 (s), 131.5 (s), $130.5(\mathrm{~d}, J=9.7 \mathrm{~Hz}), 128.5(\mathrm{~d}, J=11.7 \mathrm{~Hz})$, 122.0 (d, J = 100.5 Hz), 117.3 (s), 113.8 (s), 108.1 (s), 107.1 (s), 28.2 (d, J = 18 Hz), 11.6 (s); MS (EI) $m / z$ (rel intensity) $416\left(14,[\mathrm{M}]^{+\cdot}\right), 386$ (6), 215 (100), 201 (13), 198 (17), 185 (8), 168 (15), 154 (6), 127 (2), 77 (4); HRMS (EI) calcd for $[\mathrm{M}]^{+\cdot}\left(\mathrm{C}_{24} \mathrm{H}_{21} \mathrm{O}_{3} \mathrm{~N}_{2} \mathrm{P}\right)$ : 416.1289. found 416.1281; Anal. calcd for $\mathrm{C}_{24} \mathrm{H}_{21} \mathrm{O}_{3} \mathrm{~N}_{2} \mathrm{P}$ : C, 69.23; H, 5.08; N, 6.73. found: $\mathrm{C}, 69.04 ; \mathrm{H}$, $5.09 ; \mathrm{N}, 6.76$.

$\left(S_{\mathrm{P}}, S_{\mathrm{P}}\right)-(-)-(E)$-ethene-1,2-diylbis[methyl(phenyl)phosphine] dioxide (46): ${ }^{15,17}$ To a mixed solution of $45(0.5 \mathrm{mmol})$ in $\mathrm{CH}_{2} \mathrm{Cl}_{2}(4 \mathrm{~mL})$ was added solution $\mathbf{6 b}$
$(0.025 \mathrm{mmol})$ in $\mathrm{CH}_{2} \mathrm{Cl}_{2}(1 \mathrm{~mL})$. The resulting solution was refluxed for $16 \mathrm{~h}$. Solvent was evaporated and the crude residue was purified using flash chromatography (n-hexane-ethyl acetate-methanol 5:2:0.5 then $\mathrm{CH}_{2} \mathrm{Cl}_{2}$-methanol 10:1) to afford the title compound as a white crystalline powder, $95 \%$ yield. $[\alpha]_{\mathrm{D}}^{20}-255.0 \mathrm{deg}$ ( c $1, \mathrm{CH}_{2} \mathrm{Cl}_{2}$ ); $\mathrm{mp}=238-239{ }^{\circ} \mathrm{C}$; IR $(\mathrm{KBr}): \nu$ 3054, 2989, 2904, 1838, 1590, 1482, 1437, 1301, 1177, 1113, 1025, 894, 882, 754, 740, 692, $484 \mathrm{~cm}^{-1} ;{ }^{31} \mathrm{P}$ NMR (200 MHz, $\left.\mathrm{CDCl}_{3}\right): \delta 26.4 ;{ }^{1} \mathrm{H}$ NMR (500 $\left.\mathrm{MHz}, \mathrm{CDCl}_{3}\right)$, (second order spectrum): $\delta$ 7.68-7.62 (m, 4H), 7.53-742 (m, 6H), $7.33(\mathrm{t}$, $2 \mathrm{H}, J_{A X}+J_{B X}=50 \mathrm{~Hz}$ ), 1.83 (filled-in doublet, $6 \mathrm{H}$, line separation $=13.1 \mathrm{~Hz}) ;{ }^{13} \mathrm{C} \mathrm{NMR}$ (125 $\left.\mathrm{MHz}, \mathrm{CDCl}_{3}\right)$, (second order spectrum, only the central of multiplet signals are listed): $\delta$ 141.8 (6 lines), 132.2 (s), 132 (6 lines), 130.0 (3 lines), 128.9 (3 lines), 16.7 (6 lines); MS (ESI) $m / z$ (rel intensity) $305\left(25,[\mathrm{M}+\mathrm{H}]^{+}\right)$, $327\left(100,[\mathrm{M}+\mathrm{Na}]^{+}\right)$; HRMS (ESI) calcd for $[\mathrm{M}+\mathrm{Na}]^{+}\left(\mathrm{C}_{16} \mathrm{H}_{18} \mathrm{O}_{2} \mathrm{P}_{2} \mathrm{Na}\right)$ : 327.0674. found 327.0690 .

\section{2,2-Diphenyl-3-vinyl-2,5-dihydrofuran}

Brown oil (98\%). IR (film): $\nu$ 3427, 3059, 3026, 2925, 1765, 1682, 1598, 1490, 1447, 1226, $1179,1064,758,700 \mathrm{~cm}^{-1} ;{ }^{1} \mathrm{H}$ NMR $(500$ $\left.\mathrm{MHz} \mathrm{CDCl}_{3}\right) \delta 4.11(\mathrm{q}, 1 \mathrm{H}, J=7.1 \mathrm{~Hz}), 5.10$ $(\mathrm{dd}, 1 \mathrm{H}, J=11.2,0.8 \mathrm{~Hz}), 5.31(\mathrm{dd}, 1 \mathrm{H}, J$ $=17.7,0.8 \mathrm{~Hz}), 6.16-6.18(\mathrm{~m}, 1 \mathrm{H}), 6.20-6.27$ (m, 1H), 7.10-7.40 (m, 10H); ${ }^{13} \mathrm{C}$ NMR (125 $\left.\mathrm{MHz}, \mathrm{CDCl}_{3}\right) \delta 171.1,143.6,143.3,129.7$, $127.9,127.8,124.8,117.5,94.5,60.3$; MS (EI) $\mathrm{m} / \mathrm{z}$ (rel intensity) $248\left(15,[\mathrm{M}]^{+\cdot}\right), 229(8)$, 215 (9), 205 (18), 204 (12), 203 (19), 191 (13), 189 (10), 183 (15), 182 (22), 172 (11), 171 (77), 165 (17), 157 (18), 143 (15), 141 (10), 129 (10), 128 (22), 115 (23), 105 (100), 97 (14), 95 (9), 91 (34), 83 (11), 77 (43), 71 (16), 69 (15), 57 (20), 55 (14), 51 (14), 43 (41), 41 (12), 39 (9); HRMS (() calcd for $\mathrm{E}(\mathrm{I})$ : ); found: calcd for $[\mathrm{M}]^{+}$ $\left(\mathrm{C}_{18} \mathrm{H}_{16} \mathrm{O}\right)$ : 248.1201. found: 248.1196 .

1-[(4-methylphenyl)sulfonyl]-2,3,6,7-tetrahydro-1 $H$-azepine (48): ${ }^{19}$ Colorless solid (99\%). IR (KBr): $\nu$ 3030, 2942, 2899, 2855, 1657, 1596, 1450, 1332, 1286, 1162, 910, 816, $712 \mathrm{~cm}^{-1} ;{ }^{1} \mathrm{H}$ NMR $\left(200 \mathrm{MHz}, \mathrm{CDCl}_{3}\right)$ $\delta 2.28(\mathrm{~m}, 4 \mathrm{H}), 2.39(\mathrm{~s}, 3 \mathrm{H}), 3.25(\mathrm{~m}, 4 \mathrm{H})$, $5.72(\mathrm{~m}, 2 \mathrm{H}), 7.25(\mathrm{~d}, 2 \mathrm{H}, J=8.2 \mathrm{~Hz}), 7.64$

\footnotetext{
17 K. M. Pietrusiewicz, W. Wiśniewski, M. Zabłocka, Tetrahedron 1989, 45, 337.

18 Fürstner, A.; Ackermann, L.; Gabor, B.; Goddard, R.; Lehmann, C. W.; Mynott, R.; Stelzer, F. Thiel, O. R. Chem. Eur. J. 2001, $7,3236-3253$.

19 Grela, K.; Kim, M. Eur. J. Org. Chem. 2003, 963-966.
} 
$(\mathrm{d}, 2 \mathrm{H}, J=8.2 \mathrm{~Hz}) ;{ }^{13} \mathrm{C}$ NMR $(500 \mathrm{MHz}$, $\left.\mathrm{CDCl}_{3}\right) \delta 21.5,29.948 .2,126.9,129.5,130.1$, 136.2, 142.9; MS (EI) $\mathrm{m} / \mathrm{z}$ (rel intensity) 251 (5, [M] $\left.{ }^{+\cdot}\right), 223(2), 184(6), 155$ (4), 105 (2), 91 (19), 96 (16), 77 (1), 65 (13),42 (100); HRMS (EI) calcd for $[\mathrm{M}]^{+\cdot}\left(\mathrm{C}_{13} \mathrm{H}_{17} \mathrm{O}_{2} \mathrm{NS}\right)$ : 251.0980 . found 2251.0979 .

6-Hexyl-3,6-dimethyl-5,6-dihydro-2 $H$ -pyran-2-one (50): ${ }^{20}$ Colourless oil $(99 \%)$. IR (film): $\nu$ 2932, 2858, 1721, 1454, 1436, 1380, 1360, 1177, 1116, $986 \mathrm{~cm}^{-1} ;{ }^{1} \mathrm{H}$ NMR (500 $\left.\mathrm{MHz}, \mathrm{CDCl}_{3}\right) \delta$ 6.43-6.47 (m, 1H), 2.47 (ddd, $1 \mathrm{H}, J=2.1,14.2,18.19 \mathrm{~Hz}), 2.27(\mathrm{ddd}, 1 \mathrm{H}, J=$ $1.7,13.24,19.19 \mathrm{~Hz}), 1.91(\mathrm{~d}, 3 \mathrm{H}, J=1.8 \mathrm{~Hz})$, $1.60-1.75(\mathrm{~m}, 2 \mathrm{H}), 1.38(\mathrm{~s}, 3 \mathrm{H}), 1.25-1.32(\mathrm{~m}$, $8 \mathrm{H}), 0.88(\mathrm{t}, 3 \mathrm{H}, J=6.8 \mathrm{~Hz}) ;{ }^{13} \mathrm{C}$ NMR $(125$ $\left.\mathrm{MHz}, \mathrm{CDCl}_{3}\right) \delta 13.9,16.9,22.5,23.7,25.1$, 29.5, 31.6, 34.1, 40.8, 76.7, 77.0, 77.2, 82.3, 127.7 137.3, 165.3; MS (EI) $\mathrm{m} / \mathrm{z}$ (rel intensity) 195 (2), 145 (1), 125 (100), 113 (3), 108 (2), 97 (9), 82 (29), 69 (7), 57 (15), 43 (22).

3-Methyl-5-propyl-2(5H)-furanone (51): ${ }^{20,21}$ Colourless oil $(76 \%)$. IR (film): $\nu$ 3080, 2962, 2933, 2876, 1755, 1660, 1455, 1341, 1093, $989 \mathrm{~cm}^{-1}$; MS (EI) $\mathrm{m} / z$ (rel intensity) $140\left(14,[\mathrm{M}]^{+\cdot}\right), 127(3), 111(29), 98$ (35), 97 (50), 83 (4), 69 (49) 55 (57), 53 (5), 43 (43), 41 (100); ${ }^{1} \mathrm{H}$ NMR $\left(500 \mathrm{MHz}, \mathrm{CDCl}_{3}\right) \delta$ $7.03(\mathrm{~s}, 1 \mathrm{H}), 4.87-4.89(\mathrm{~m}, 1 \mathrm{H}), 1.91(\mathrm{~s}, 3 \mathrm{H})$, $1.39-1.72(\mathrm{~m}, 4 \mathrm{H}), 0.96(\mathrm{t}, 3 \mathrm{H}, J=7.3 \mathrm{~Hz})$. ${ }^{13} \mathrm{C}$ NMR $\left(125 \mathrm{MHz}, \mathrm{CDCl}_{3}\right) \delta 10.6,13.8$, 18.4, 35.5, 76.7, 77.0, 77.2, 80.8, 129.8, 148.7, 147.3; HRMS (ESI) calc for [M] ${ }^{+\cdot}\left(\mathrm{C}_{8} \mathrm{H}_{12} \mathrm{O}_{2}\right)$ : 140.08378. found 140.08373 .

11-Methyldodec-10-en-1-ol (52): Colorless oil (99\%). IR (film): $\nu$ 3338, 2964, 2927, 2855, 1452, 1377, 1262, 1057, 801, $701 \mathrm{~cm}^{-1}$; ${ }^{1} \mathrm{H}$ NMR $\left(500 \mathrm{MHz}, \mathrm{CDCl}_{3}\right) \delta$ 1.25-1.38 $(\mathrm{m}, 14 \mathrm{H}), 1.46(\mathrm{~s}, 1 \mathrm{H}), 1.59(\mathrm{~s}, 3 \mathrm{H}), 1.69(\mathrm{~s}$, $3 \mathrm{H}), 1.92-1.99(\mathrm{~m}, 2 \mathrm{H}), 3.63(\mathrm{t}, 6.6, J=2 \mathrm{H}$ $\mathrm{Hz}), 5.09-5.14(\mathrm{~m}, 1 \mathrm{H}) ;{ }^{13} \mathrm{C}$ NMR $(125 \mathrm{MHz}$, $\left.\mathrm{CDCl}_{3}\right) \delta 131.1,124.9,63.0,32.8,29.9,29.8$, 29.6, 29.5, 29.4, 29.3, 28.0, 25.7, 17.6; MS (EI) $\mathrm{m} / \mathrm{z}$ (rel intensity) $198\left(10,[\mathrm{M}]^{+\cdot}\right), 180(7)$, 124 (10), 123 (7), 109 (14), 96 (26), 95 (38), 83 (14), 82 (51), 81 (30), 70 (14), 69 (100), 68 (22), 67 (27), 57 (12), 56 (27), 55 (29), 41 (31), 39 (4); HRMS (EI) calcd for [M] ${ }^{+\cdot}\left(\mathrm{C}_{13} \mathrm{H}_{26} \mathrm{O}\right)$ : 198.1984. found: 198.1986; Anal. calcd for $\mathrm{C}_{13} \mathrm{H}_{26} \mathrm{O}: \mathrm{C} 78.72, \mathrm{H}$ 13.21. found: C 78.76, H 13.34 .

\section{1-(6-Methylhept-5-enyl)cyclohexanol} (53): Colorless oil (99\%): IR (film): $\nu$ 3392, 2932, 2857, 1449, 1378, 1262, 1169, 966, $835 \mathrm{~cm}^{-1} ;{ }^{1} \mathrm{H}$ NMR $\left(500 \mathrm{MHz}, \mathrm{CDCl}_{3}\right) \delta$ $1.23-1.58(\mathrm{~m}, 17 \mathrm{H}), 1.60(\mathrm{~s}, 3 \mathrm{H}), 1.68(\mathrm{~s}, 3 \mathrm{H})$, $1.99(\mathrm{q}, 2 \mathrm{H}, J=6.5 \mathrm{~Hz}), 5.09-5.14(\mathrm{~m}, 1 \mathrm{H}) ;{ }^{13} \mathrm{C}$ NMR $\left(125 \mathrm{MHz}, \mathrm{CDCl}_{3}\right) \delta 131.2,124.7,71.4$, 42.4, 37.4, 30.5, 28.0, 25.8, 25.7, 22.5, 22.2, 17.6; MS (EI) $m / z$ (rel intensity) $192\left(26,\left[\right.\right.$ M-18] $\left.{ }^{+}\right)$ 149 (32), 136 (30), 135 (20), 122 (16), 121 (18), 111 (10), 110 (15), 109 (40), 108 (19), 107 (19), 99 (68), 97 (13), 96 (48), 95 (38), 93 ()17, 82 (91), 81 (100), 79 (21), 69 (40), 67 (40), 56 (7), 55 (41), 53 (7), 43 (8), 41 (25); HRMS (ESI) calcd for $[\mathrm{M}+\mathrm{Na}]^{+}\left(\mathrm{C}_{14} \mathrm{H}_{26} \mathrm{ONa}\right)$ : 233.1876 . found: 233.1887 .

7-[1-(tert-Butyl)-1,1-dimethylsilyl]oxy-2-heptenyl acetate (54): Colorless oil, $(E):(Z)=$ 7.9:1 (67\%). IR (film): $\nu 3474,2931,2858,2256$, $1743,1673,1520,1472,1463,1384,1362,1255$, $1102,1025,970,911,836,776,734 \mathrm{~cm}^{-1} ;{ }^{1} \mathrm{H}$ NMR $\left(500 \mathrm{MHz}, \mathrm{CDCl}_{3}\right) \delta 0.05(\mathrm{~s}, 6 \mathrm{H}), 0.89$ $(\mathrm{s}, 9 \mathrm{H}), 1.35-1.47(\mathrm{~m}, 2 \mathrm{H}), 1.49-1.54(\mathrm{~m}, 2 \mathrm{H})$, $2.06(\mathrm{~s}, 3 \mathrm{H}), 2.07-2.10(\mathrm{~m}, 2 \mathrm{H}), 3.60(\mathrm{t}, 2 \mathrm{H}, \mathrm{J}$ $=6.4 \mathrm{~Hz}), 4.51(\mathrm{~d}, 2 \mathrm{H}, J=6.5 \mathrm{~Hz}), 5.52-5.60$ $(\mathrm{m}, 1 \mathrm{H}), 5.73-5.80(\mathrm{~m}, 1 \mathrm{H}) ;{ }^{13} \mathrm{C}$ NMR $(125$ $\left.\mathrm{MHz}, \mathrm{CDCl}_{3}\right) \delta 170.8,136.3,123.9,65.2,62.9$, $32.2,31.9,25.9,25.1,21.0,18.3,-5.3$; MS (EI) $\mathrm{m} / \mathrm{z}$ (rel intensity) $229\left(1,[\mathrm{M}-57]^{+}\right) 207(1)$, 170 (2), 169 (15), 159 (7), 141 (6), 118 (10), 117 (100), 101 (4), 95 (59), 93 (4), 89 (3), 75 (18), 67 (9), 43 (6), 41 (2); HRMS (ESI) calcd for $[\mathrm{M}+\mathrm{Na}]^{+}\left(\mathrm{C}_{15} \mathrm{H}_{30} \mathrm{O}_{3} \mathrm{SiNa}\right): 309.1856$. found: 309.1859

Isopropyl 8-(acetyloxy)-6-methyl-6-octenoate (55): Colorless oil $(E):(Z)=2.3: 1$ (37\%). IR (film): $\nu$ 3454, 2981, 2939, 2870, 1732, 1454, 1375, 1235, 1181, 1146, 1109, 1026, $959,824 \mathrm{~cm}^{-1} ;{ }^{1} \mathrm{H}$ NMR $\left(500 \mathrm{MHz}, \mathrm{CDCl}_{3}\right)$ $\delta \quad 1.23(\mathrm{~d}, 6 \mathrm{H}, J=6.2 \mathrm{~Hz}), 1.37-1.51(\mathrm{~m}$, $2 \mathrm{H}), 1.57-1.65(\mathrm{~m}, 2 \mathrm{H}), 1.69(\mathrm{~d}, 3 \mathrm{H}$, isomer $(E), J=0.3 \mathrm{~Hz}), 1.74(\mathrm{~d}, 3 \mathrm{H}$, isomer $(\mathrm{Z}), J$ $=0.9 \mathrm{~Hz}), 2.04(\mathrm{~s}, 3 \mathrm{H}$, isomer $(E)), 2.10(\mathrm{~s}$, $3 \mathrm{H}$, isomer $(\mathrm{Z})), 2.27(\mathrm{t}, 2 \mathrm{H}, J=7.4 \mathrm{~Hz})$, $4.55(\mathrm{~d}, 2 \mathrm{H}$, isomer $(\mathrm{Z}), J=7.3 \mathrm{~Hz}), 4.58(\mathrm{~d}$, $2 \mathrm{H}$, isomer $(E), J=7.1 \mathrm{~Hz}$ ), 5.01 (hept, $1 \mathrm{H}$, $J=6.2 \mathrm{~Hz}), 5.30-5.37(\mathrm{~m}, 1 \mathrm{H}),{ }^{13} \mathrm{C}$ NMR $\left(125 \mathrm{MHz}, \mathrm{CDCl}_{3}\right) \delta 173.1,171.1,141.9,118.5$, 67.4, 61.3, 39.1, 34.5, 26.9, 24.6, 21.8, 21.0, 16.2; MS (EI) $m / z$ (rel intensity) $213\left(1,[\mathrm{M}-43]^{+}\right)$, 197 (7), 196 (37), 179 (4), 171 (4), 155 (19), 154 (100), 153 (40), 139 (4), 137 (48), 136 (10), 135 (22), 127 (7), 125 (10), 111 (8), 110 (11),

\footnotetext{
${ }^{20}$ Grela, K.; Trynowski, M.; Bieniek, M. Tetrahedron Lett. 2002, 43, 9055-9059.

21 Ma, S.; Yu, Z.; Wu, S. Tetrahedron 2001, 57, $1585-1588$.
} 
109 (33), $108(13), 97(10), 95(39), 94(80), 93$ (41), 81 (28), 79 (31), 71 (9.5), 68 (18), 67 (18), 55 (13), 43 (43), 41 (13), 39 (4); HRMS (ESI) calcdf for $[\mathrm{M}+\mathrm{Na}]^{+}\left(\mathrm{C}_{14} \mathrm{H}_{24} \mathrm{O}_{4} \mathrm{Na}\right): 279.1567$. found: 279.1580 .

2-[1-[(4-Methylphenyl)sulfonyl]tetrahydro-4(1 $H$ )-pyridinyliden]ethyl acetate (56): Colorless oil (47\%). IR (film): $\nu$ 2910, 2846, 1737, 1598, 1466, 1338, 1234, 1166, 1096, $1025,931,817,728 \mathrm{~cm}^{-1}$; ${ }^{1} \mathrm{H}$ NMR $(500 \mathrm{MHz}$, $\left.\mathrm{CDCl}_{3}\right) \delta 2.01(\mathrm{~s}, 3 \mathrm{H}), 2.28-2.33(\mathrm{~m}, 3 \mathrm{H})$, 2.38-2.43 (m, 4H), 3.02-3.09 (m, 4H), $4.51(\mathrm{~d}$, $2 \mathrm{H}, J=7.2 \mathrm{~Hz}), 5.35(\mathrm{t}, 1 \mathrm{H}, J=7.2 \mathrm{~Hz})$, 7.29-7.33 (m, 2H), 7.62-7.65 (m, 2H); ${ }^{13} \mathrm{C}$ NMR $\left(125 \mathrm{MHz}, \mathrm{CDCl}_{3}\right) \delta 170.7,143.5,139.8$, 133.4, 129.6, 127.6, 118.7, 59.9, 47.4, 46.9, 35.0, 27.9, 21.4, 20.9; MS (EI) $m / z$ (rel intensity) 323 (0.2), 279 (0.5), 263 (13), 184 (6), 168 (6), 155 (18), 109 (11), 108 (100), 107 (12), 91 (49), 81 (41), 79 (9), 77 (7), 65 (13), 43 (26), 42 (25), 41 (11), 39 (8); HRMS (ESI) calcd for $[\mathrm{M}+\mathrm{Na}]^{+}$ $\left(\mathrm{C}_{16} \mathrm{H}_{21} \mathrm{NO}_{4} \mathrm{SNa}\right)$ : 346.1084. found: 346.1097; Anal. calcd for $\mathrm{C}_{16} \mathrm{H}_{21} \mathrm{NO}_{4} \mathrm{~S}$ : C 59.42, $\mathrm{H}$ 6.54, N 4.33, S 9.91. found: C 59.40, H 6.57, N 4.17, $\mathrm{S} 9.98$.

1-[(4-Methylphenyl)sulfonyl]-4-[2-(1,1,1-trimethylsilyl)ethylidene]piperidine (57): ${ }^{22}$ Colorless oil (5\%). IR (film): $\nu$ 3087, 2957, 2852, 1727, 1673, 1627, 1598, 1582, 1516, 1484, $1340,1271,1258,1165,1105,1040,995,950$, 847, 799, $728 \mathrm{~cm}^{-1}$; ${ }^{1} \mathrm{H}$ NMR $(500 \mathrm{MHz}$, $\left.\mathrm{CDCl}_{3}\right) \delta 0.10(2,9 \mathrm{H}), 1.45(\mathrm{~d}, 2 \mathrm{H}, J=6.0$ $\mathrm{Hz}), 1.56(\mathrm{~s}, 3 \mathrm{H}), 2.30-2.43(\mathrm{~m}, 4 \mathrm{H}), 3.05-3.12$ $(\mathrm{m}, 4 \mathrm{H}), 5.18-5.26(\mathrm{~m}, 1 \mathrm{H}), 7.31-7.37(\mathrm{~m}$, 2H), 7.64-7.69 (m, 2H); ${ }^{13} \mathrm{C}$ NMR (100 MHz, $\left.\mathrm{CDCl}_{3}\right) \delta 152.2,143.4,130.2,129.6,127.6$ $112.2,47.8,47.5,38.6,32.9,21.9,0.2 ; \mathrm{MS}$ (EI) $\mathrm{m} / z$ (rel intensity) $337\left(17,[\mathrm{M}]^{+\cdot}\right), 323(16)$, 308 (15), 257 (17), 256 (100), 228 (9), 180 (28), 168 (14), 155 (8), 149 (21), 109 (5), 91 (13), 73 (39), 59 (5), 45 (2). HRMS (EI) calcd for [M] ${ }^{+}$. $\left(\mathrm{C}_{17} \mathrm{H}_{27} \mathrm{NO}_{2} \mathrm{SSi}\right): 337.1532$. found: 337.1536 .

3,4-dimethyl-1-[(4-methylphenyl)sulfonyl]-2,5-dihydro-1 $H$-pyrrole (59): ${ }^{23} \mathrm{MS}$ (EI) $\mathrm{m} / \mathrm{z}$ (rel intensity) 251 (3), 250 (4), 236 (18), 186 (1), 170 (1), 155 (23), 139 (3), 96 (100), 94 (22), 91 (69), 81 (14), 80 (15), 67 (9), 65 (32), 63 (7), 55 (9), 53 (10), 41 (40), 39 (29).

\section{4-Methyl-2,2-diphenyl-3-vinyl-2,5-}

-dihydrofuran (62): ${ }^{18}$ MS (EI) $m / z$ (rel intensity) 262 (6), 247 (4), 229 (9), 215 (6), 205 (14), 202 (7), 186 (12), 185 (100), 171 (11), 165 (11), 157 (6), 141 (10), 129 (18), 115 (21), 105 (55), 91 (38), 79 (29), 77 (68), 65 (6), 51 (34), 43 (39), 41 (5), 39 (21).

1,3,4-trimethyl-cyclohex-3-en-1-ol (63): ${ }^{24}$ MS (EI) $m / z$ (rel intensity) 140 (2), 125 (10), 122 (31), 106 (5), 107 (66), 105 (6), 97 (10), 91 (17), 83 (19), 82 (39), 79 (15), 69 (6), 67 (98), 65 (8), 58 (29), 55 (40), 53 (18), 51 (10), 43 (100), 41 (43), 39 (40), 38 (3).

2-Phenyl-3,6-dihydro-2 $H$-pyran (64): ${ }^{25}$ Colourless oil $(90 \%)$. IR (thin film): $\nu$ 3339, 3034, 2926, 2895, 2830, 1723, 1452, 1388, 1179, 1090, 762, 699, $657 \mathrm{~cm}^{-1}$; ${ }^{1} \mathrm{H}$ NMR $(200 \mathrm{MHz}$, $\left.\mathrm{CDCl}_{3}\right) \delta$ 7.45-7.25 (m, 5H), 6.00-5.88 (m, $1 \mathrm{H}), 5.87-5.77(\mathrm{~m}, 1 \mathrm{H}), 4.57(\mathrm{dd}, 1 \mathrm{H}, \mathrm{J}=$ $12.2,4 \mathrm{~Hz}), 4.43-4.33(\mathrm{~m}, 2 \mathrm{H}), 2.41-2.18(\mathrm{~m}$, $2 \mathrm{H}) ;{ }^{13} \mathrm{C} \mathrm{NMR}\left(50 \mathrm{MHz}, \mathrm{CDCl}_{3}\right) \delta$ 142.5, 128.3, 127.4, 126.4, 125.8, 124.4, 75.6, 66.5, 32.8; HRMS (EI) calcd for $[\mathrm{M}]^{+\cdot}\left(\mathrm{C}_{11} \mathrm{H}_{12} \mathrm{O}\right)$ : 160.0888. found 160.0893 .

1-[(4-Methylphenyl)sulphonyl]-3-(2-methyl-1-propenyl)-2,5-dihydro-1H-pyrrole (65): ${ }^{18}$ White solid (99\%). ${ }^{1} \mathrm{H}$ NMR $\left(200 \mathrm{MHz}, \mathrm{CDCl}_{3}\right) \delta 7.71(\mathrm{~d}, 2 \mathrm{H}, J=8.2 \mathrm{~Hz})$, 7.31 (d, 2H, $J=7.9 \mathrm{~Hz}), 5.60$ (bs, 1H), 5.37 (bs, $1 \mathrm{H}), 4.22(\mathrm{~d}, 2 \mathrm{H}, \mathrm{J}=3.3 \mathrm{~Hz}), 4.12(\mathrm{bs}, 2 \mathrm{H})$, $2.41(\mathrm{~s}, 3 \mathrm{H}), 1.74(\mathrm{~d}, 6 \mathrm{H}, \mathrm{J}=6.4 \mathrm{~Hz}) ;{ }^{13} \mathrm{C} \mathrm{NMR}$ $\left(50 \mathrm{MHz} \mathrm{CDCl}_{3}\right) \delta 143.3,137.8,136.2,129.7$, 127.4, 120.8, 117.9, 56.3, 54.5, 27.3, 21.6, 19.9; HRMS (EI) calcd for $[\mathrm{M}]^{+\cdot}\left(\mathrm{C}_{15} \mathrm{H}_{19} \mathrm{O}_{2} \mathrm{NS}\right)$ : 277.1136. found: 277.1132 .

2,5,2',5'-tetrahydro-[3,3']bifuranyl (67): White solid (55\%). ${ }^{1} \mathrm{H}$ NMR (500 MHz, $\left.\mathrm{CDCl}_{3}\right) \delta 5.67(\mathrm{bs}, 2 \mathrm{H}), 4.80-4.73(\mathrm{~m}, 8 \mathrm{H}) ;{ }^{13} \mathrm{C}$ NMR $\left(125 \mathrm{MHz}, \mathrm{CDCl}_{3}\right) \delta 131.8,122.9,76.3$, 75.1; HRMS (EI) calcd for [M] ${ }^{+\cdot}\left(\mathrm{C}_{8} \mathrm{H}_{10} \mathrm{O}_{2}\right)$ : 138.0681. found: 138.0683 .

4-[(Allyloxy)methyl]-3-methylene-3,6-dihydro-2H-pyran (68): Yellow oil (24\%). ${ }^{1} \mathrm{H}$ $\operatorname{NMR}\left(200 \mathrm{MHz}, \mathrm{CDCl}_{3}\right) \delta$ 6.05-5.85 (m, 2H), $5.36(\mathrm{q}, 1 \mathrm{H}, J=1.7 \mathrm{~Hz}), 5.28-5.22(\mathrm{~m}, 1 \mathrm{H})$, $5.20(\mathrm{q}, 1 \mathrm{H}, J=1.3 \mathrm{~Hz}), 5.08(\mathrm{~s}, 1 \mathrm{H}), 4.88(\mathrm{~s}$, $1 \mathrm{H}), 4.34-4.28(\mathrm{~m}, 2 \mathrm{H}), 4.26(\mathrm{t}, 2 \mathrm{H}, J=1.2$ $\mathrm{Hz}), 4.18(\mathrm{q}, 2 \mathrm{H}, J=1.6 \mathrm{~Hz}), 4.03(\mathrm{dt}, 2 \mathrm{H}, J$ $=5.7,1.4 \mathrm{~Hz}) ;{ }^{13} \mathrm{C} \mathrm{NMR}\left(50 \mathrm{MHz}, \mathrm{CDCl}_{3}\right)$

\footnotetext{
${ }^{22}$ Ciufolini, M. A.; Rivera-Fortin, M. A.; Byrne, N. E. Tetrahedron Lett. 1993, 34, 3505-3508

23 Krafft, M. E.; Bonaga, L. V. R.; Wright, J. A.; Hirosawa, C. J. Org. Chem. 2002, 67, $1233-1246$.

24 Singleton, D. A.; Leung, S.-W. J. Organomet. Chem. 1997, 544, 157-162.

25 Larock, R. C.; Gong, W. H.; Baker, B. E. Tetrahedron Lett. 1989, 30, $2603-2606$.
} 
$\delta$ 138.0, 134.7, 131.5, 126.9, 117.2, 107.7, 71.3, 69.6, 69.4, 66.0; IR (thin film): $\nu$ 3345, 3084, 2855, 1727, 1647, 1614, 1451, 1342, 1270, 1126, 1081, $931 \mathrm{~cm}^{-1}$; HRMS (LSIMS) calcd for $[\mathrm{M}+\mathrm{Na}]^{+}\left(\mathrm{C}_{10} \mathrm{H}_{14} \mathrm{O}_{2} \mathrm{Na}\right)$ : 189.0886. found: 189.0900 .

\section{Procedures for recycling of the catalyst}

RCM formation of 47: In a Schlenk flask the catalyst $\mathbf{6 b}(0.025 \mathrm{~g}, 0.037 \mathrm{mmol})$ was placed. The flask was filled with argon and methylene chloride $(65 \mathrm{~mL})$ was added. The solution was cold to $0^{\circ} \mathrm{Cand}$ then a solution of the substrate $(0.374 \mathrm{~g}, 1.5 \mathrm{mmol})$ in methylene chloride $(10 \mathrm{~mL})$ was added. The resulting solution was stirred at $0{ }^{\circ} \mathrm{Cfor} 55$ minutes. The solvent was evaporated and the residue was dissolved in minimal amount of methylene chloride. The catalyst was precipitated with $n$-pentane. The crude $\mathbf{6 b}$ was purified by column chromatography $(0-20 \%$ ethyl acetate/cyclohexane). The catalyst $(0.018 \mathrm{~g}$, $72 \%$ ) was obtained as a green solid. The product 47 was formed in quantitative yield (purity $\geqslant 98 \%$ by GC).

RCM formation of 49: In a Schlenk flask the catalyst $\mathbf{6 b}(0.025 \mathrm{~g}, 0.037 \mathrm{mmol})$ was placed. The flask was filled with argon and methylene chloride $(65 \mathrm{~mL})$ was added. Then a solution of the substrate $(0.362 \mathrm{~g}, 1.5 \mathrm{mmol})$ in methylene chloride $(10 \mathrm{~mL})$ was added and the resulting solution was stirred at room temperature for 45 minutes. The solvent was evaporated and the residue was dissolved in minimal amount of methylene chloride. The catalyst was precipitated with $n$-pentane. The crude $\mathbf{6 b}$ was purified by column chromatography $(0-20 \%$ ethyl acetate/cyclohexane). The catalyst $(0.010 \mathrm{~g}, 40 \%)$ was obtained as a green solid. The product 49 was formed in quantitative yield (purity $\geqslant 98 \%$ by GC).

RCM formation of 28a: In a Schlenk flask the catalyst $\mathbf{6 b}(0.025 \mathrm{~g}, 0.037 \mathrm{mmol})$ was placed. The flask was filled with argon and methylene chloride $(65 \mathrm{~mL})$ was added.
Then a solution of $27 \mathrm{a}(0.208 \mathrm{~g}, 1.5 \mathrm{mmol})$ in methylene chloride $(10 \mathrm{~mL})$ was added under an argon atmosphere and the resulting solution was stirred at room temperatures for $1 \mathrm{~h}$. The solvent was evaporated and the residue was subject to column chromatography $(50 \%$ ethyl acetate/cyclohexane). The crude catalyst was dissolved in minimal amount of methylene chloride and it was precipitated with $n$-pentane. The catalyst $0.013 \mathrm{~g}$, (52\%) was obtained as a green solid. The product 28a was formed in quantitative yield (purity $\geqslant 98 \%$ by GC).

\section{Ab initio studies}

All the calculations were performed using Gaussian 98 (Gaussian 98, Revision A.11.4, Gaussian, Inc., Pittsburgh PA, 2002) ${ }^{26}$ on a IRIX64/Linux workstation. The structres of 2-isopropoxy styrenes were optimized using B3LYP with 6-31G** basis set. Only real values of the analytical harmonic vibrational frequencies confirmed that geometries under study correspond to the minimum-energy structures.

\subsection{Electron density distribution surface maps}

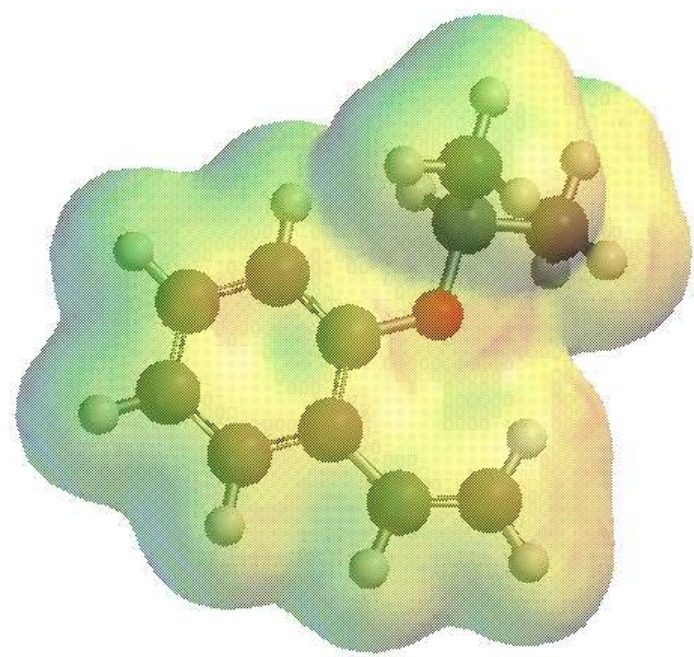

Figure 1a. 2-Isopropoxystyrene 72

${ }^{26}$ M. J. Frisch, G. W. Trucks, H. B. Schlegel, G. E. Scuseria, M. A. Robb, J. R. Cheeseman, V. G. Zakrzewski, J. A. Montgomery, Jr., R. E. Stratmann, J. C. Burant, S. Dapprich, J. M. Millam, A. D. Daniels, K. N. Kudin, M. C. Strain, O. Farkas, J. Tomasi, V. Barone, M. Cossi, R. Cammi, B. Mennucci, C. Pomelli, C. Adamo, S. Clifford, J. Ochterski, G. A. Petersson, P. Y. Ayala, Q. Cui, K. Morokuma, N. Rega, P. Salvador, J. J. Dannenberg, D. K. Malick, A. D. Rabuck, K. Raghavachari, J. B. Foresman, J. Cioslowski, J. V. Ortiz, A. G. Baboul, B. B. Stefanov, G. Liu, A. Liashenko, P. Piskorz, I. Komaromi, R. Gomperts, R. L. Martin, D. J. Fox, T. Keith, M. A. Al-Laham, C. Y. Peng, A. Nanayakkara, M. Challacombe, P. M. W. Gill, B. Johnson, W. Chen, M. W. Wong, J. L. Andres, C. Gonzalez, M. Head-Gordon, E. S. Replogle, and J. A. Pople, Gaussian, Inc. 


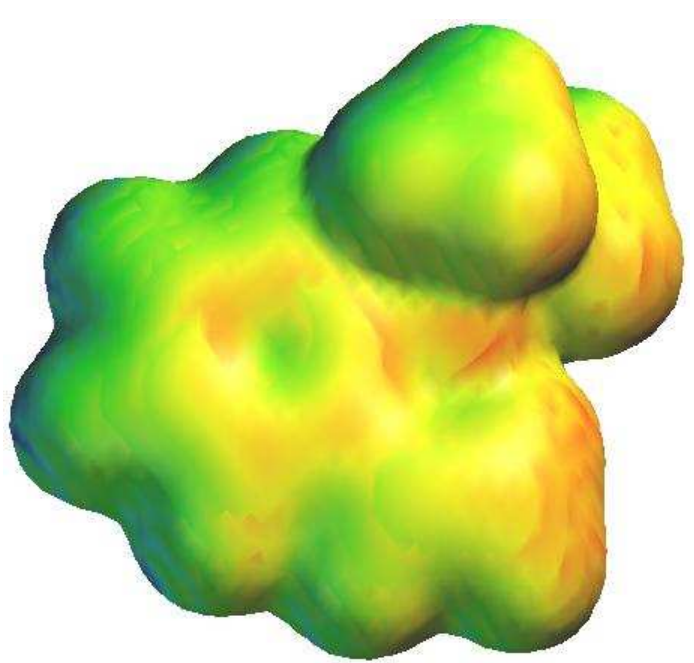

Figure 1b. 2-Isopropoxystyrene $\mathbf{7 2}$

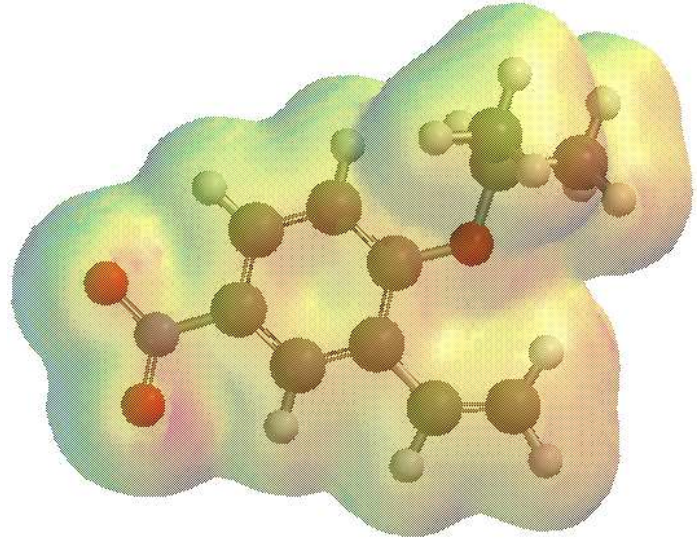

Figure 2a. 2-Isopropoxy-5-nitrostyrene $\mathbf{1 4}$

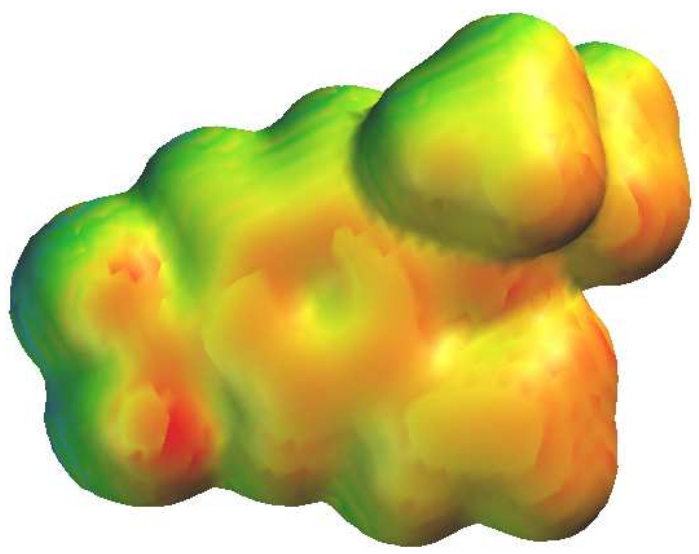

Figure 2b. 2-Isopropoxy-5-nitrostyrene $\mathbf{1 4}$

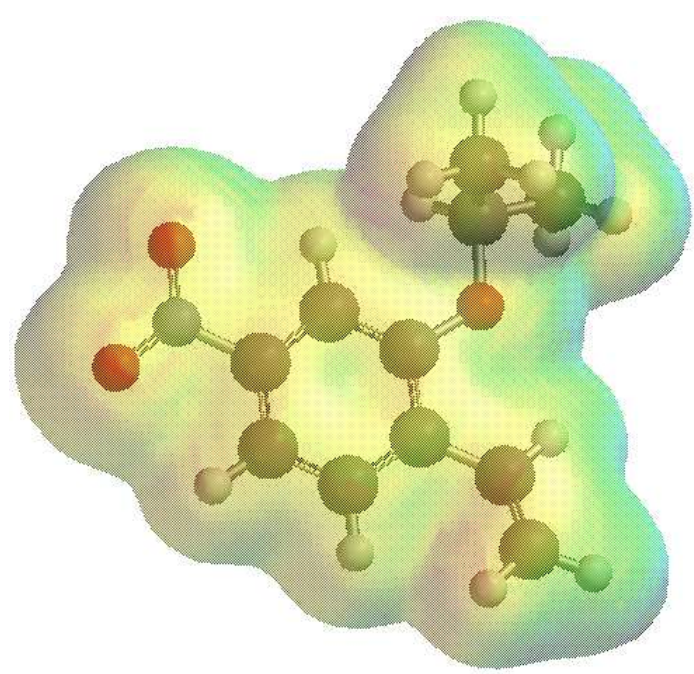

Figure 3a. 2-Isopropoxy-4-nitrostyrene 15

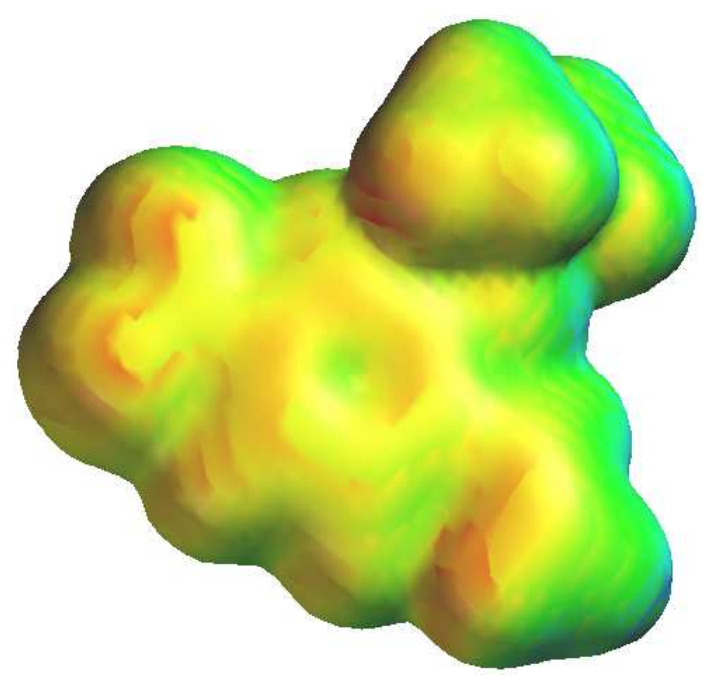

Figure 3b. 2-Isopropoxy-4-nitrostyrene 15

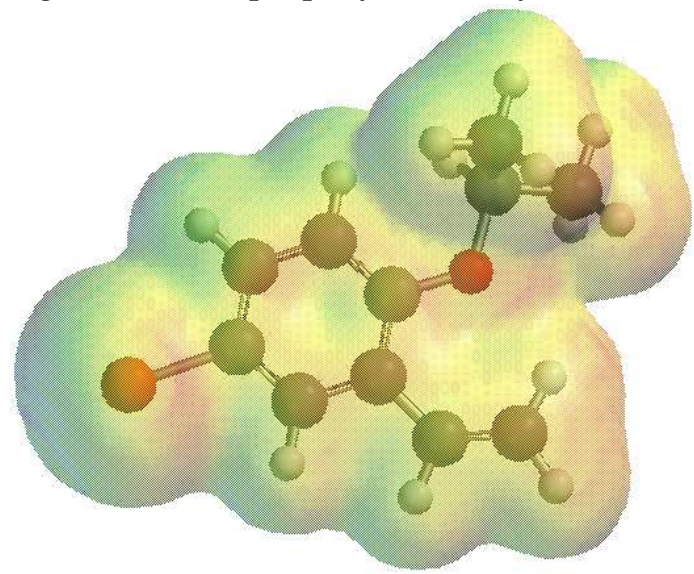

Figure 4a. 2-Isopropoxy-5-bromostyrene 


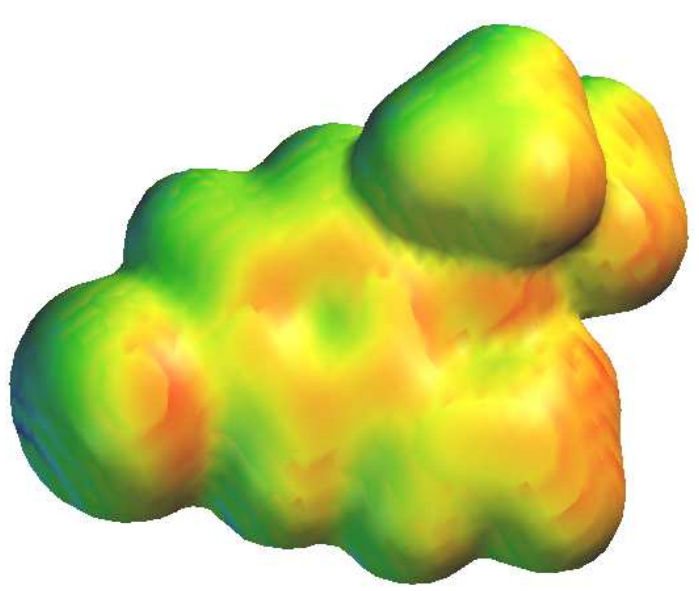

Figure 4b. 2-Isopropoxy-5-bromostyrene

\subsection{ESP charges}

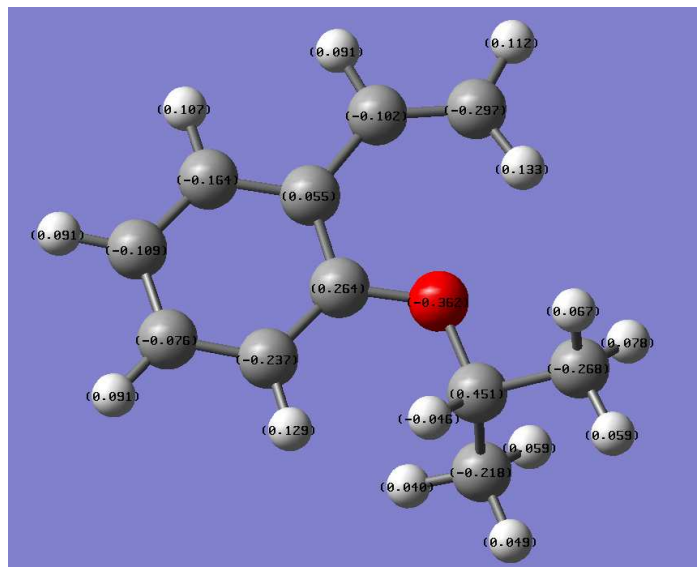

Figure 5. 2-Isopropoxystyrene $\mathbf{7 2}$

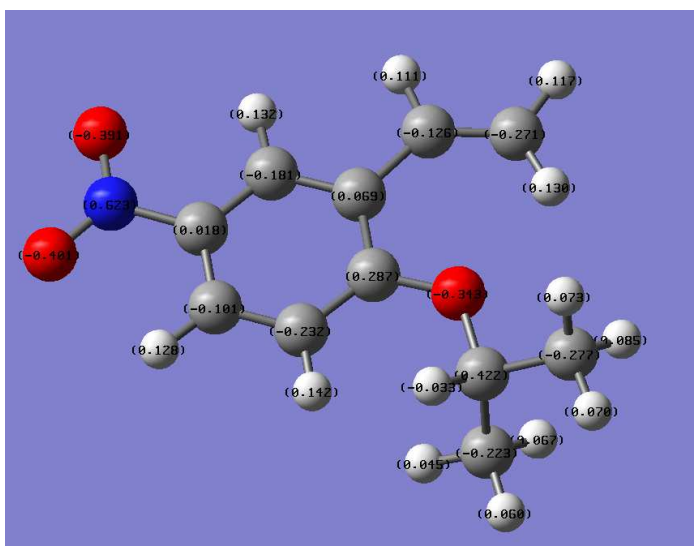

Figure 6. 2-Isopropoxy-5-nitrostyrene $\mathbf{1 4}$

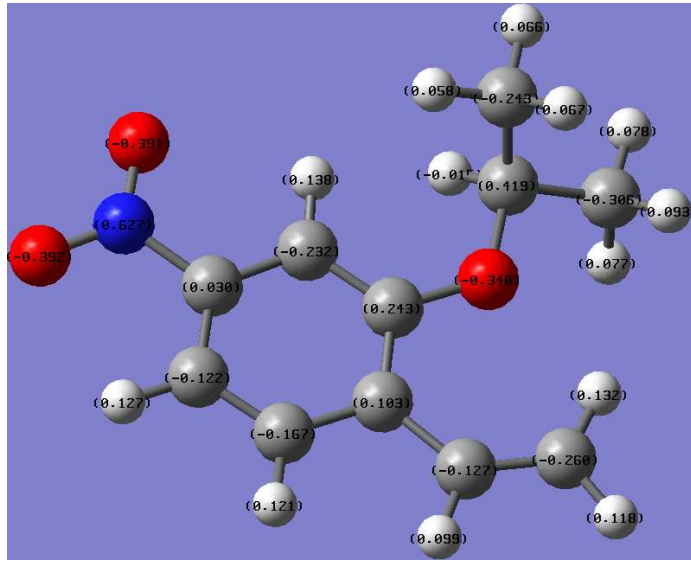

Figure 3. 2-Isopropoxy-4-nitrostyrene 15

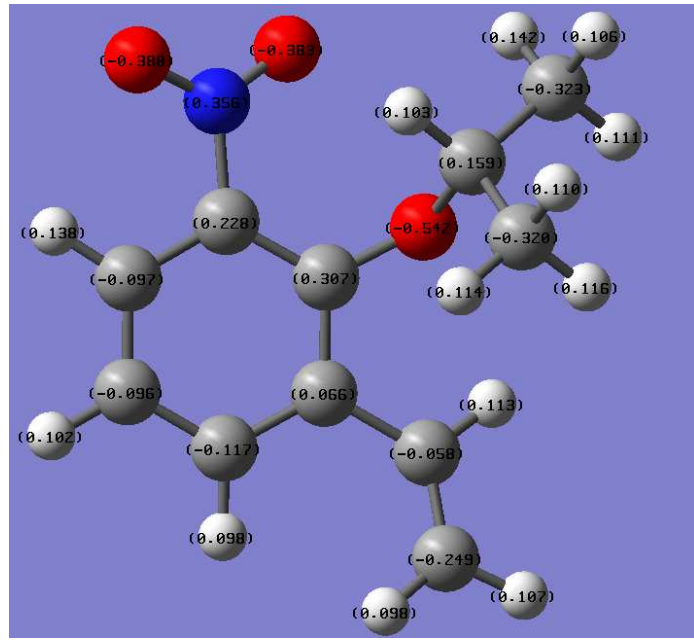

Figure 7. 2-Isopropoxy-3-nitrostyrene

\subsection{Mulliken charges}

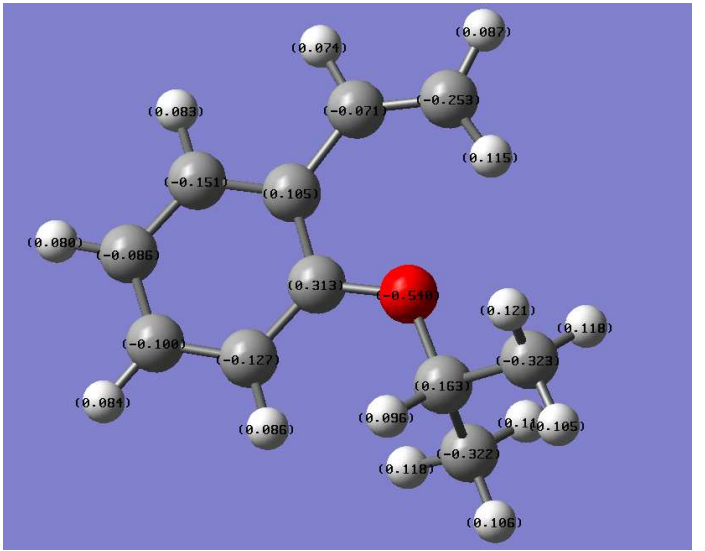

Figure 8. 2-Isopropoxystyrene $\mathbf{7 2}$ 


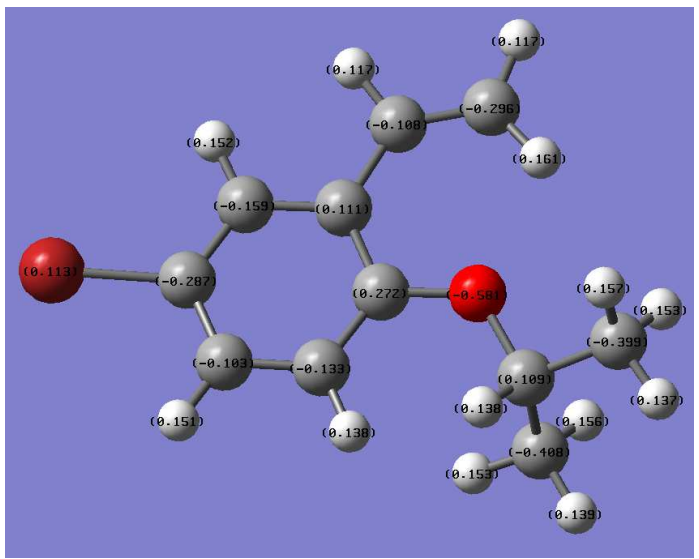

Figure 9. 2-Isopropoxy-5-bromostyrene

\section{1D and 2D NMR Spectra}




\section{Compound 6a}

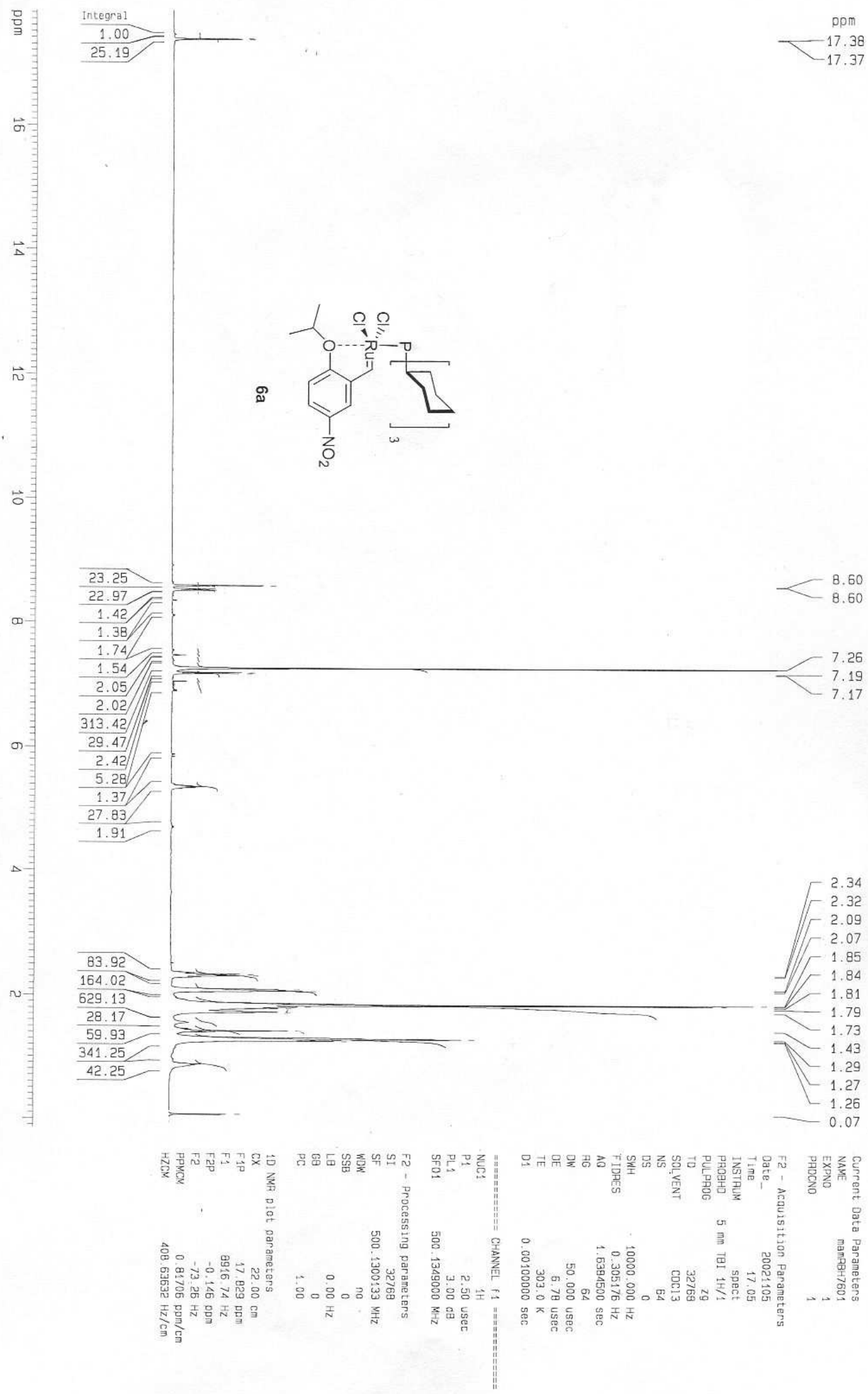



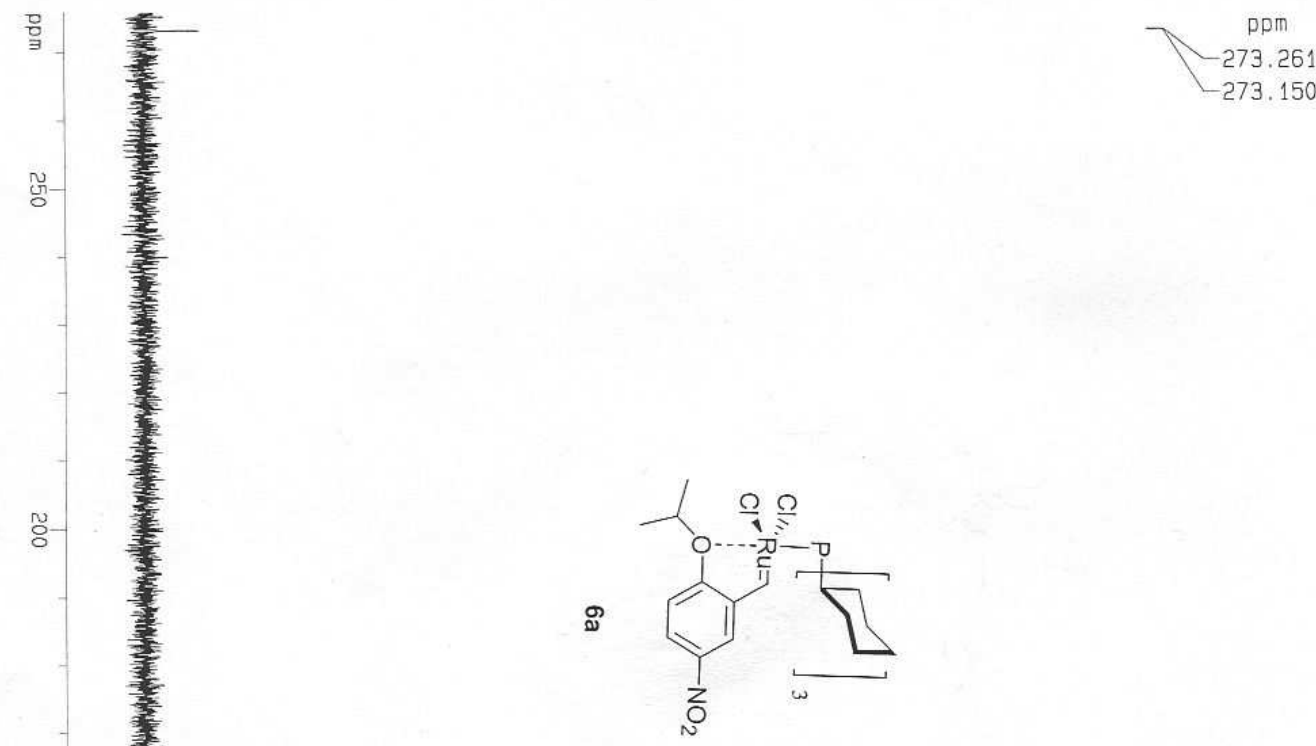

$-273.150$

药

하

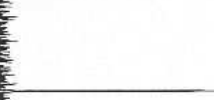

156.986

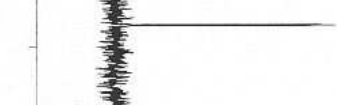

$-143.296$

143,141

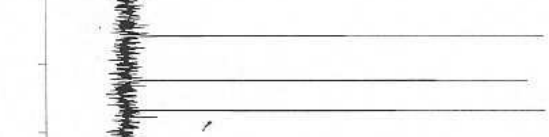

$-117.621$

$-113.215$

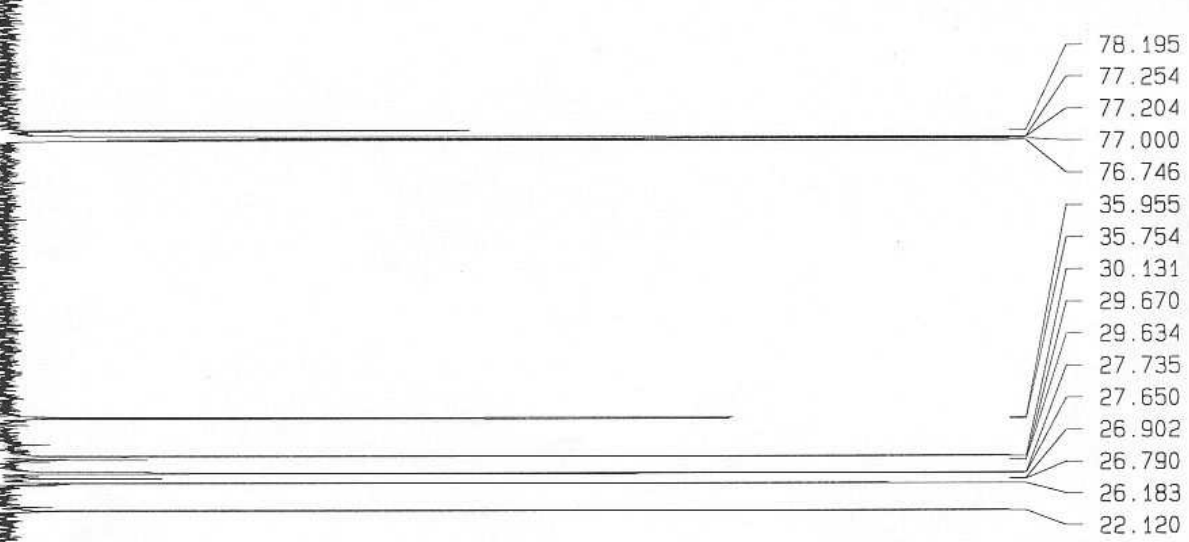

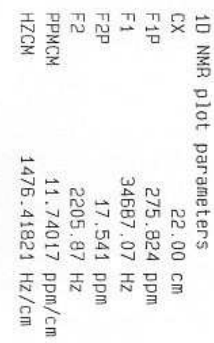

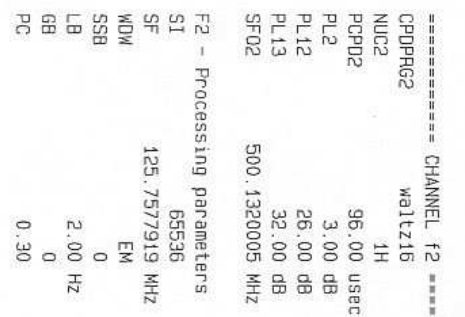

뉼물모름

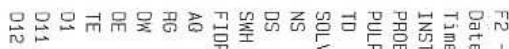

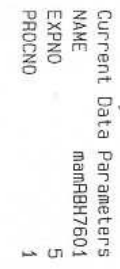




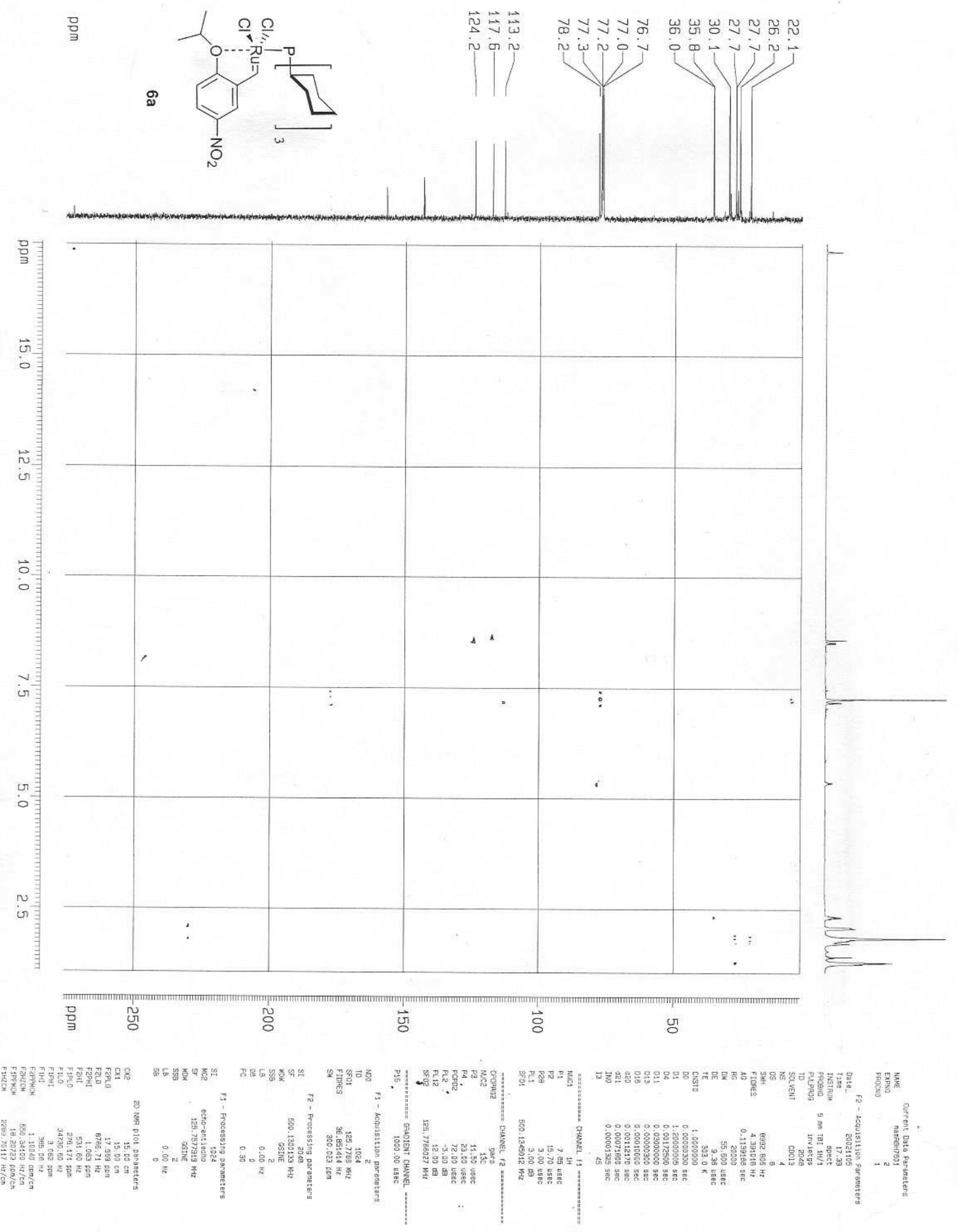




\section{Compound $6 \mathrm{~b}$}

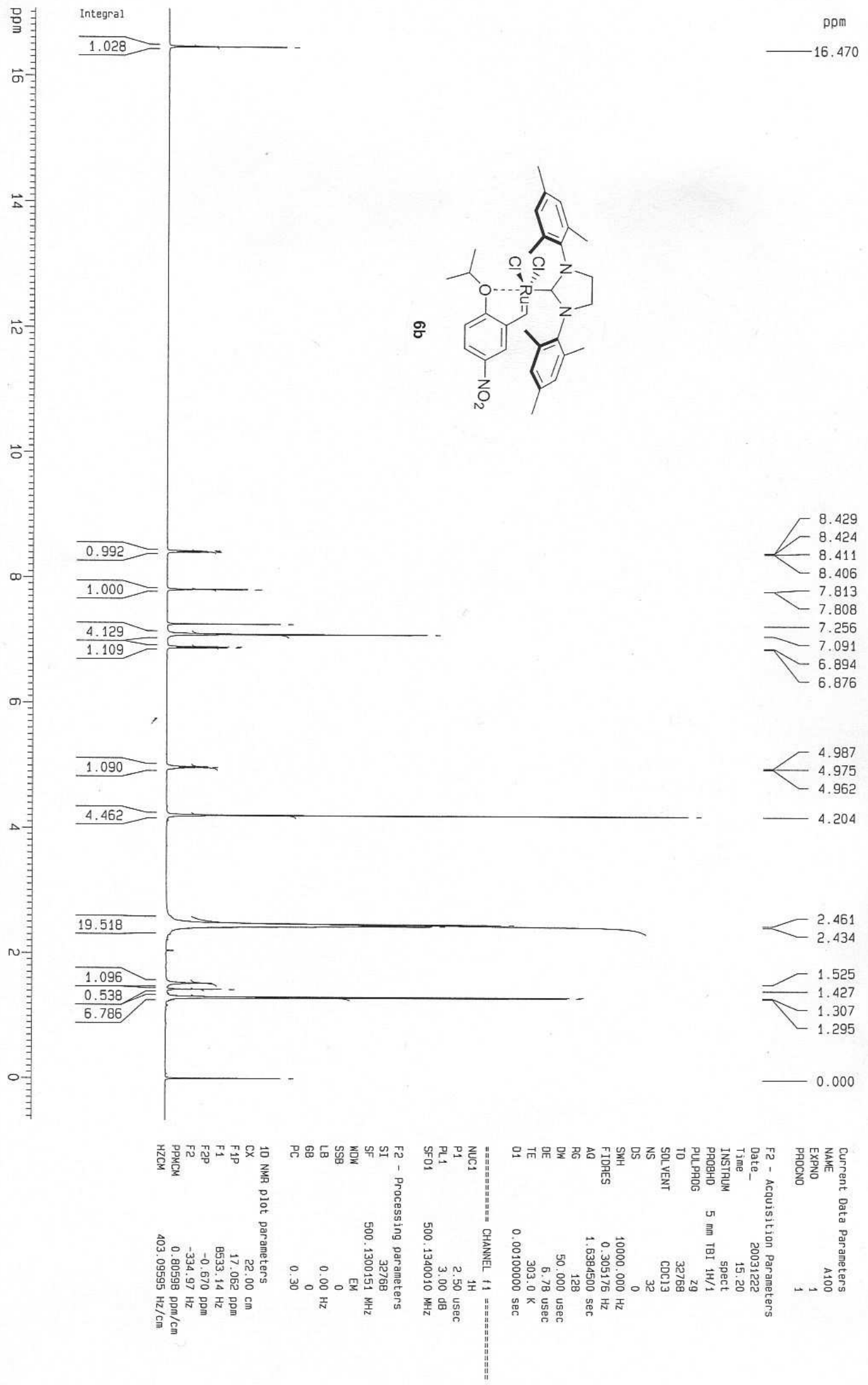



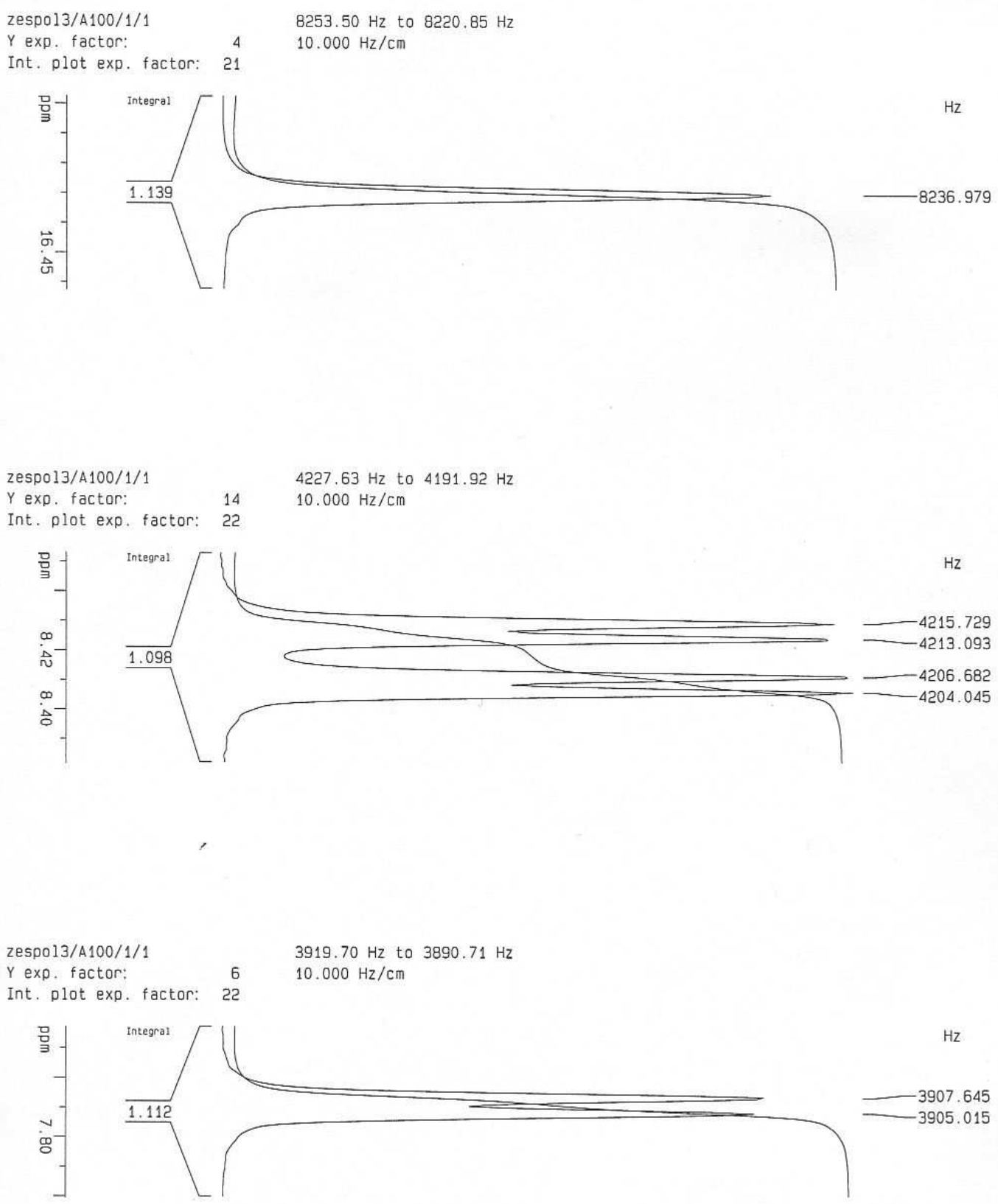

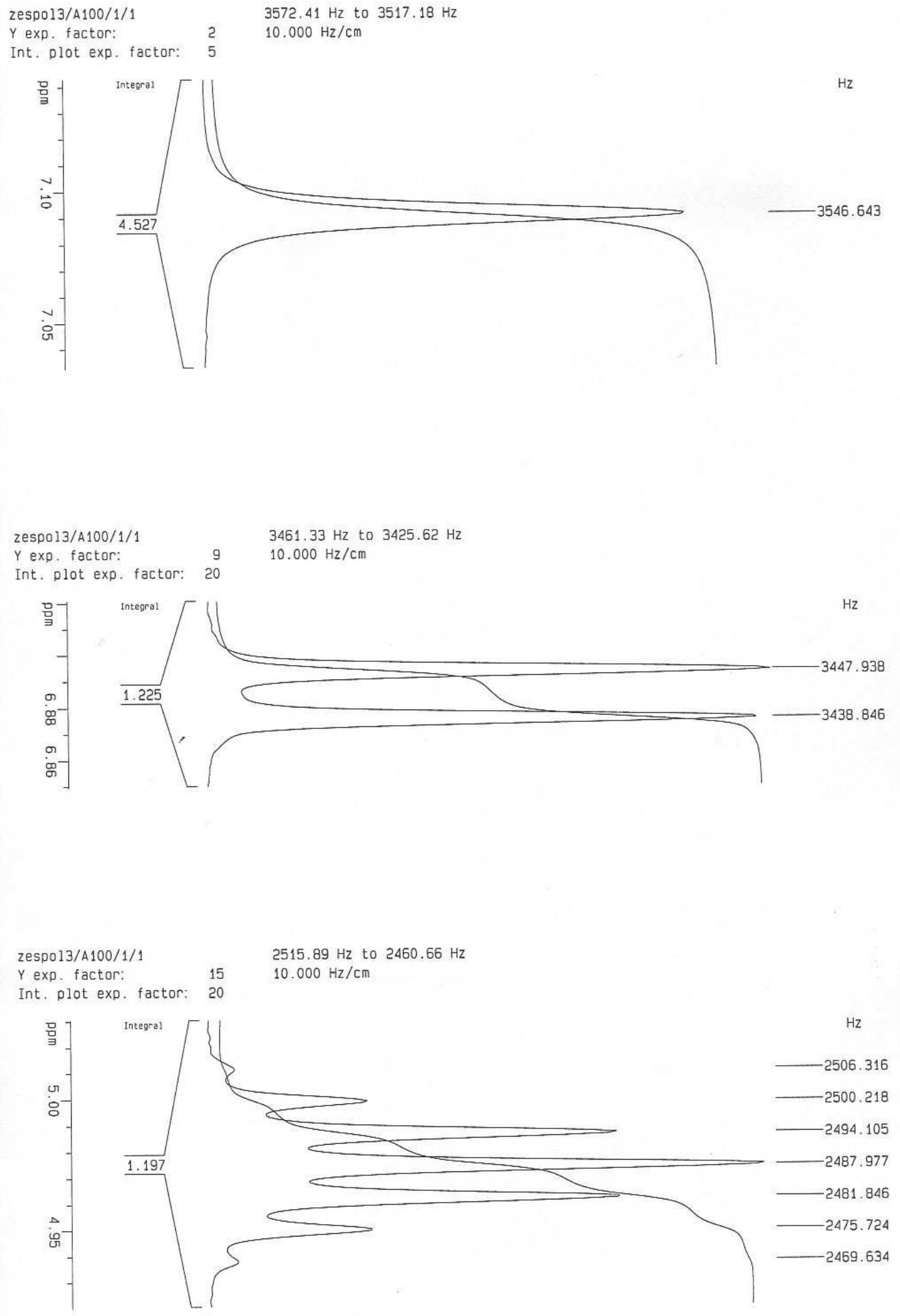


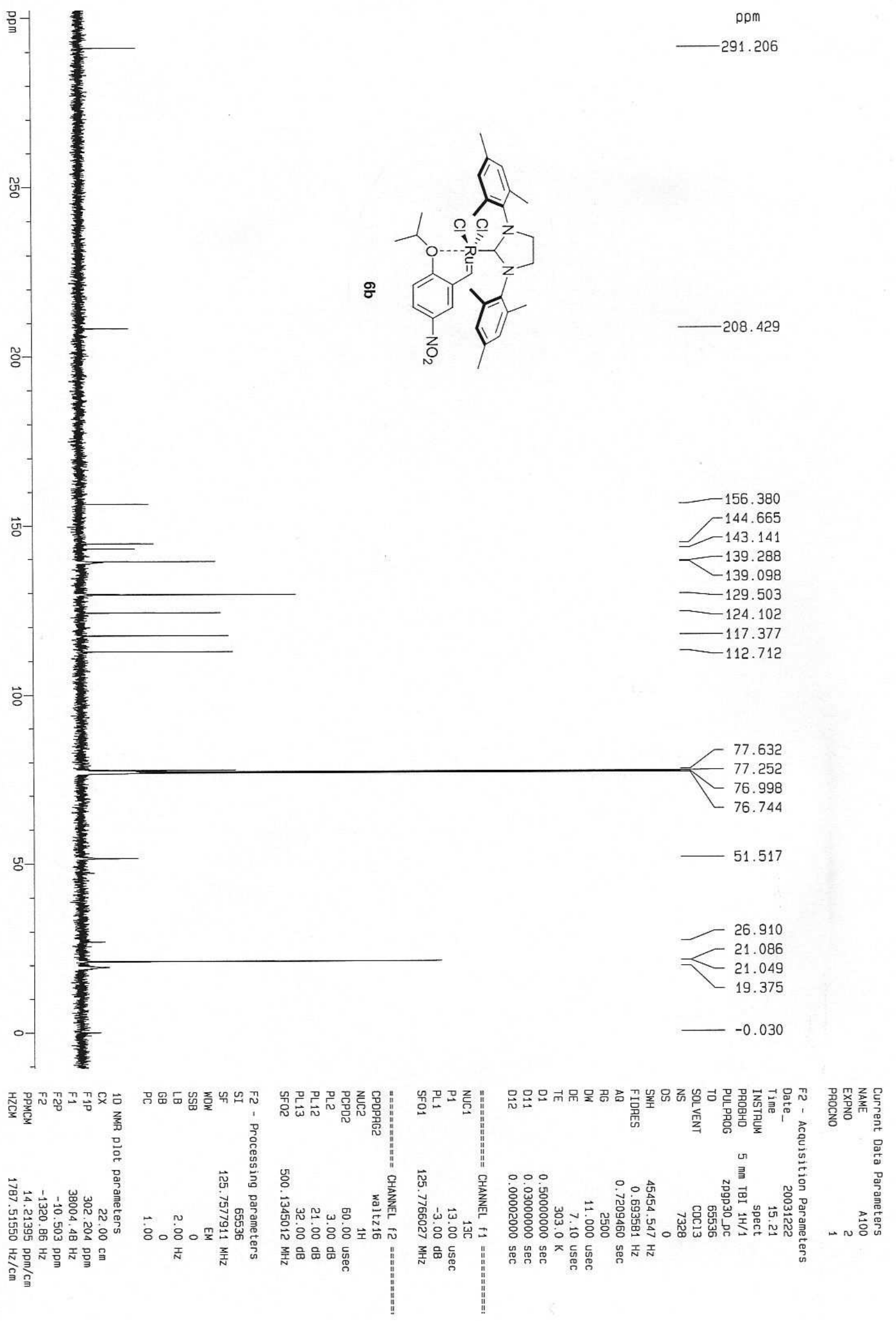




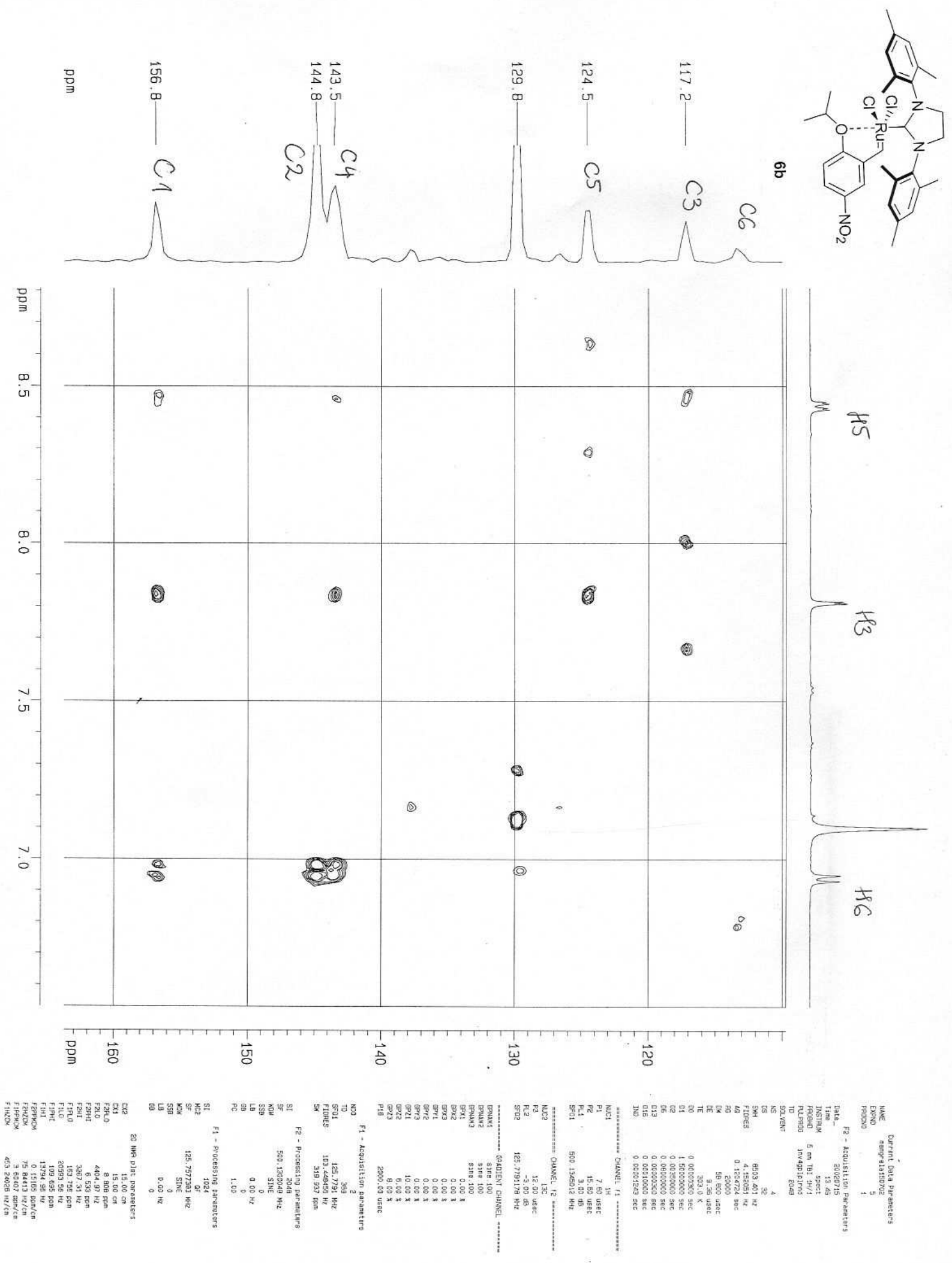


뭌

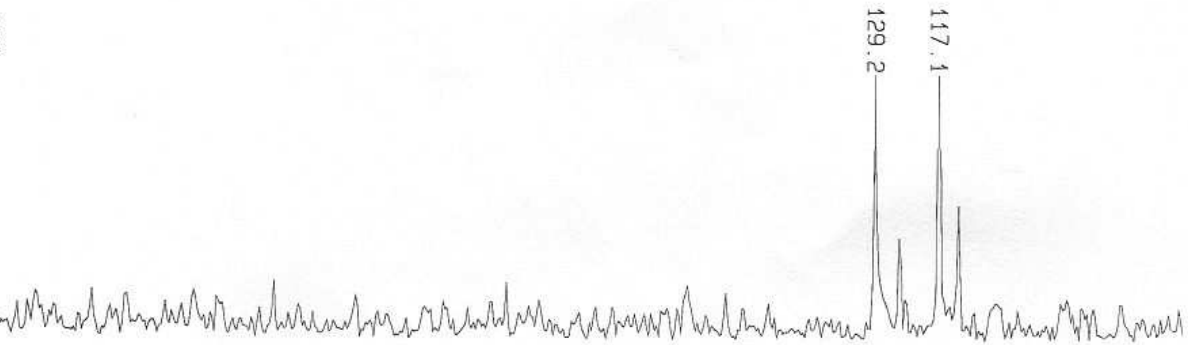
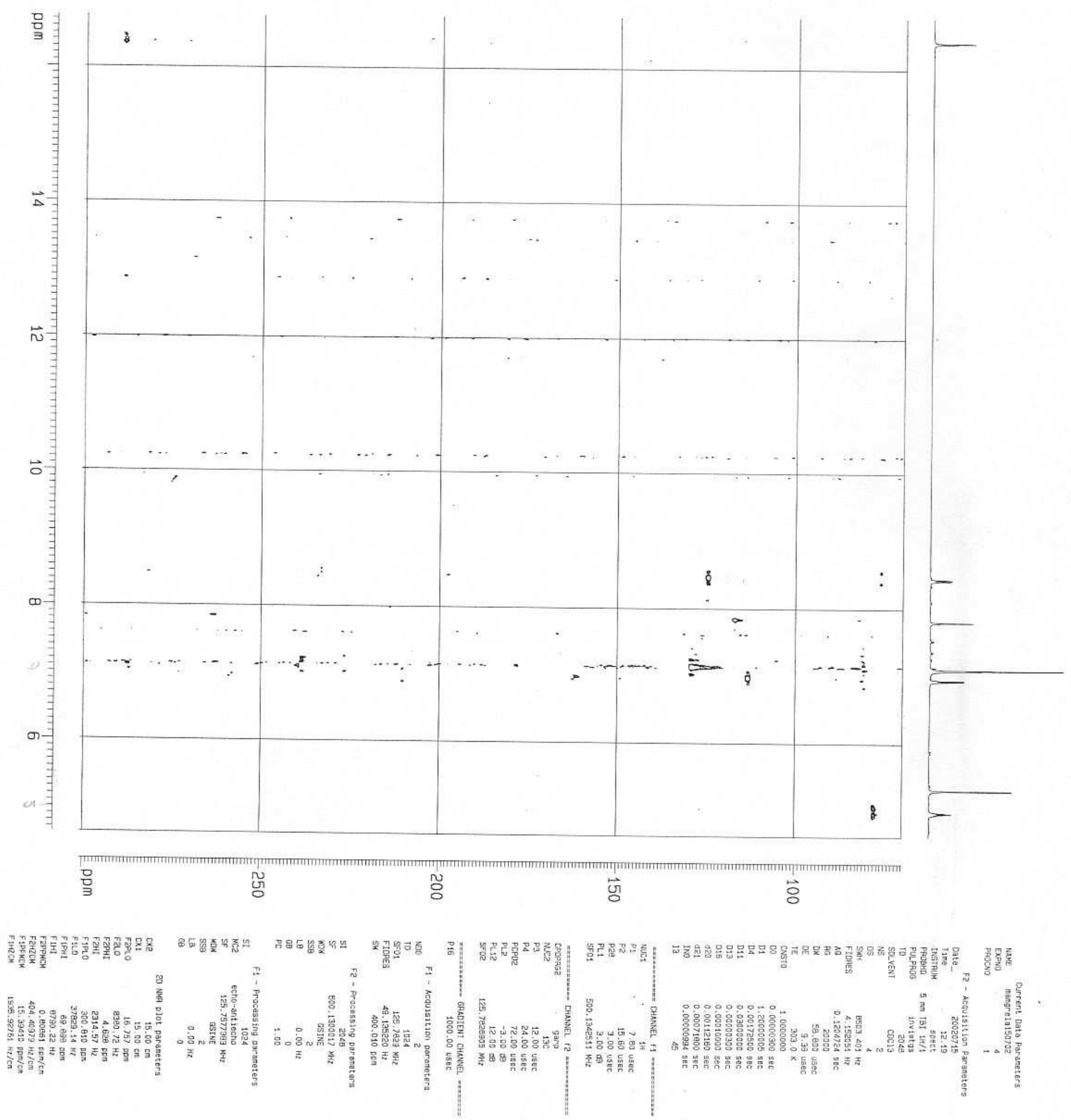


\section{Compound $7 \mathrm{~b}$}

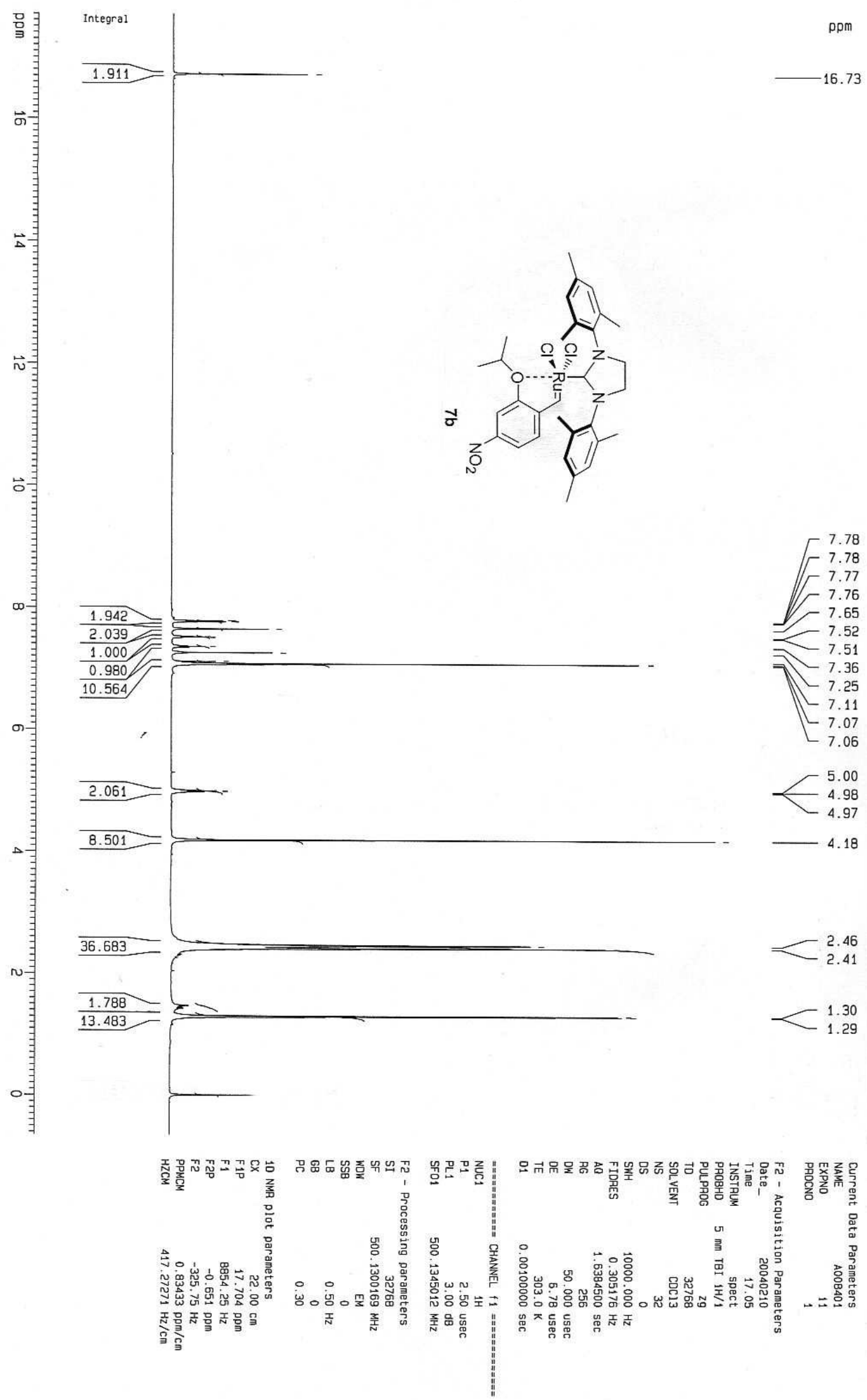



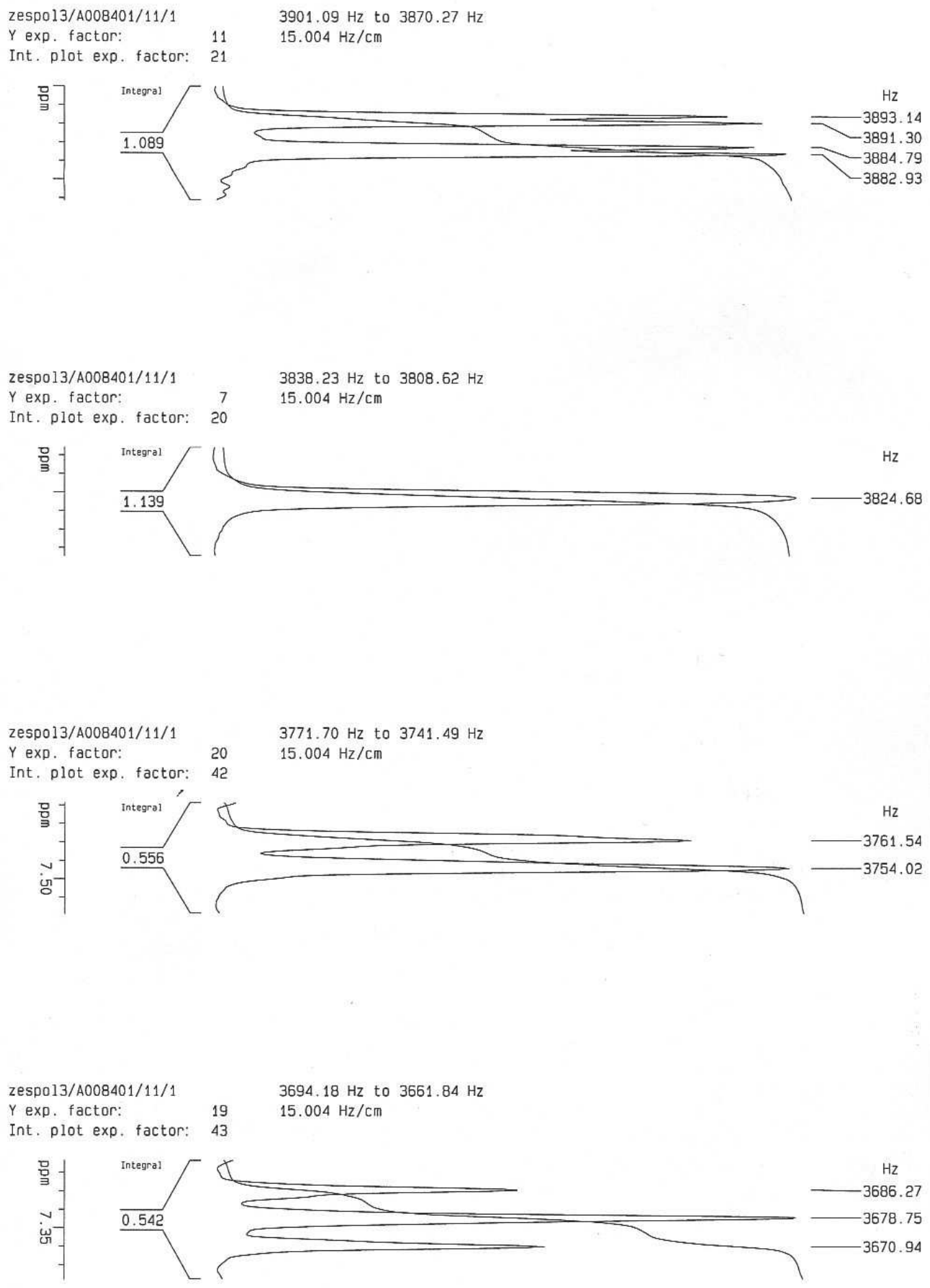


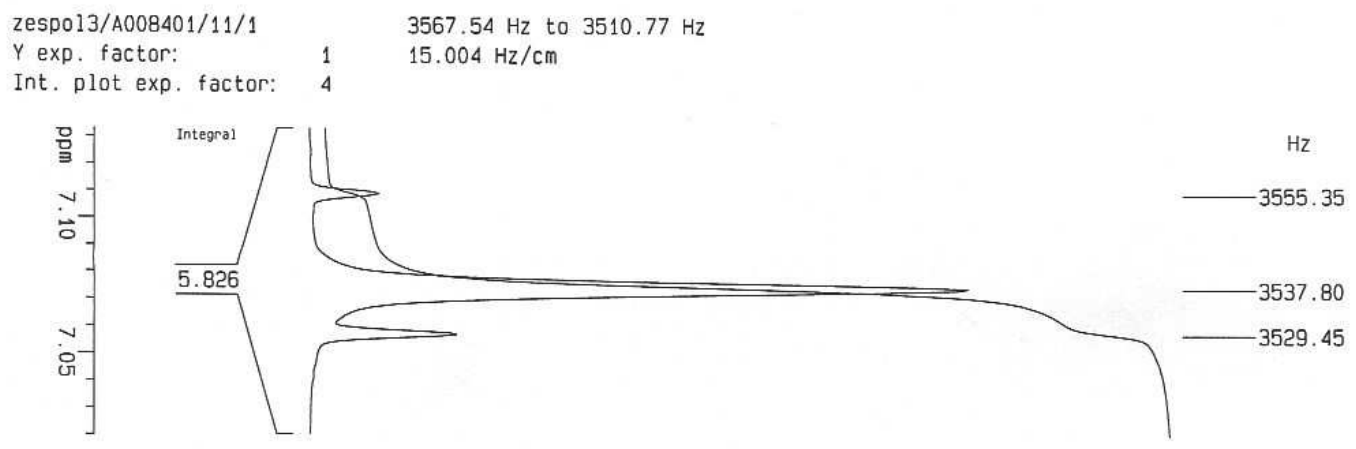

$\begin{array}{lll}\text { zespo13/A008401/11/1 } & 2516.51 \mathrm{~Hz} \text { to } 2464.33 \mathrm{~Hz} \\ \text { Y exp. factor: } & 14 & 15.004 \mathrm{~Hz} / \mathrm{cm}\end{array}$

Int. plot exp. factor: 20

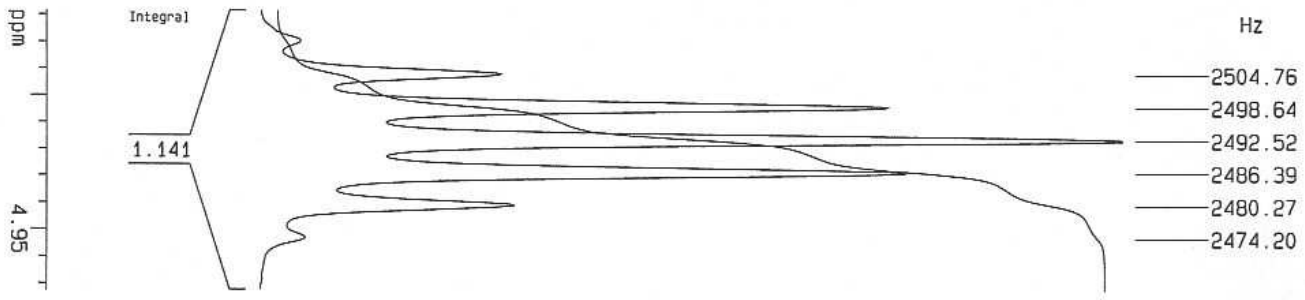

$\begin{array}{ll}\text { zespol3/A008401/11/1 } & 2117.04 \mathrm{~Hz} \text { to } 2063.02 \mathrm{~Hz} \\ \text { Y exp. factor: } & 15.004 \mathrm{~Hz} / \mathrm{cm}\end{array}$

Int. plot exp. factor: 5

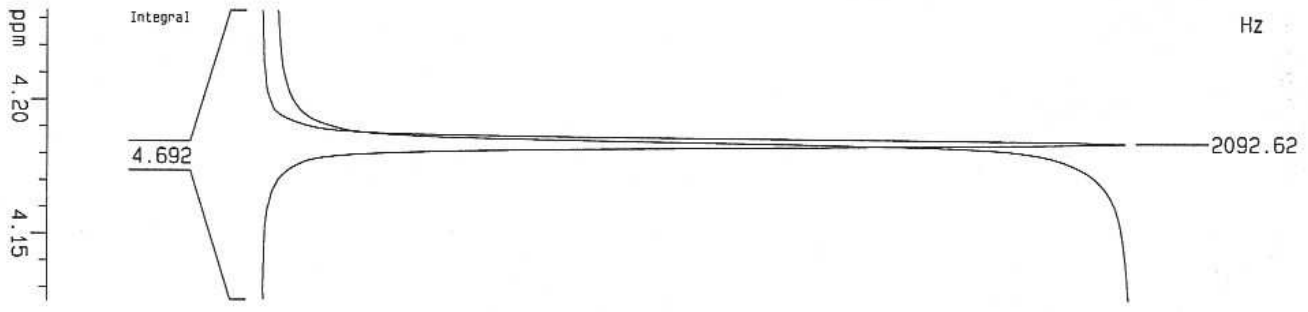



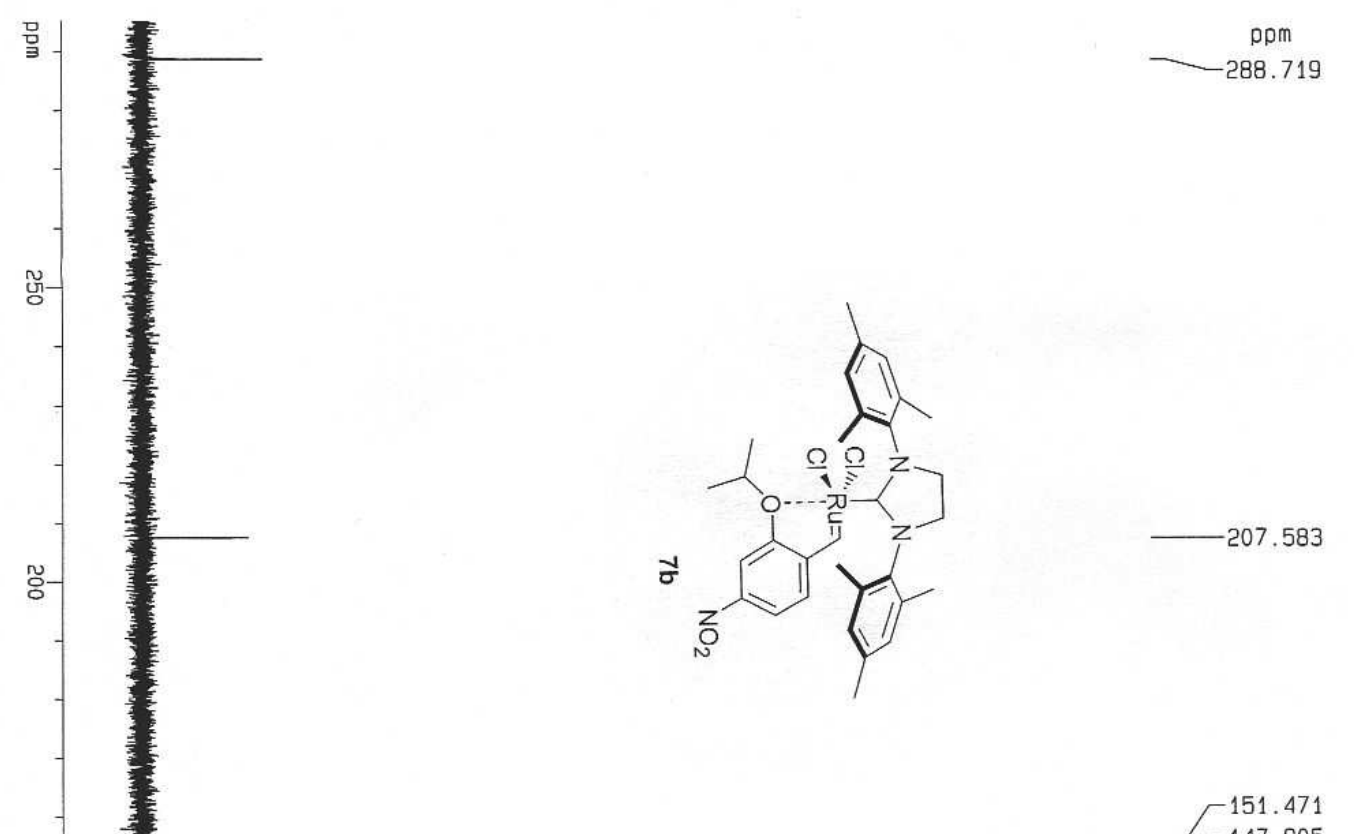

$-145.337$

$-139.114$

134.065

$-129.443$

$-128.705$

$-128.656$

$-127.593$

$-126.494$

$-121.401$

$-119.250$

$-108.200$

77.261

77.007

76.753

nᄂ -

51.532

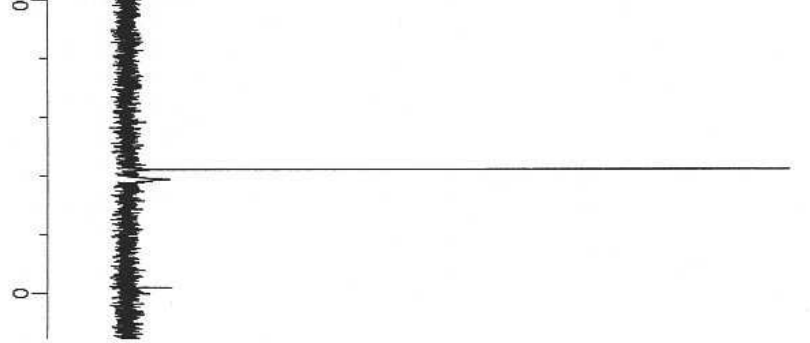

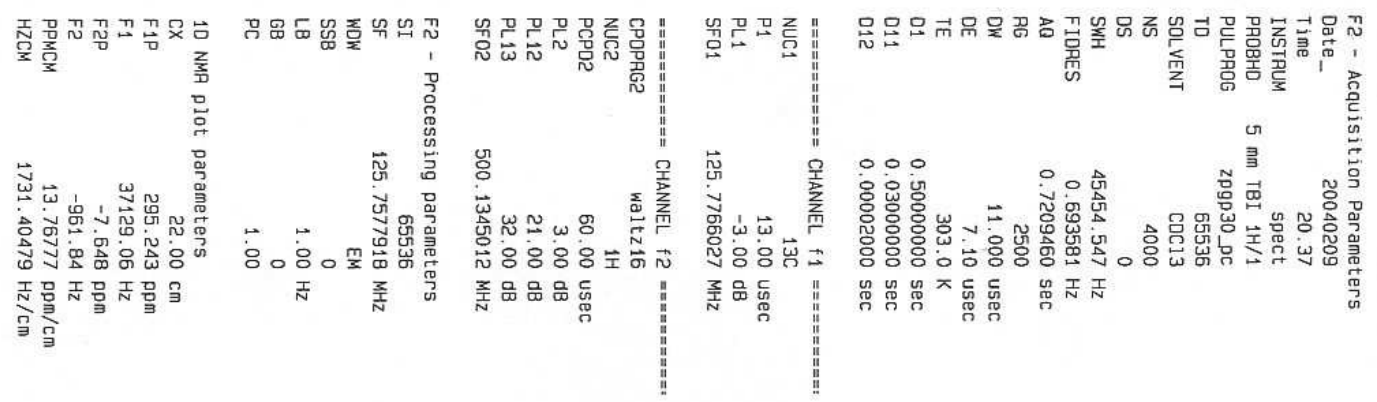

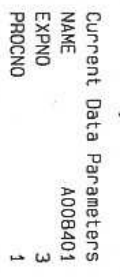




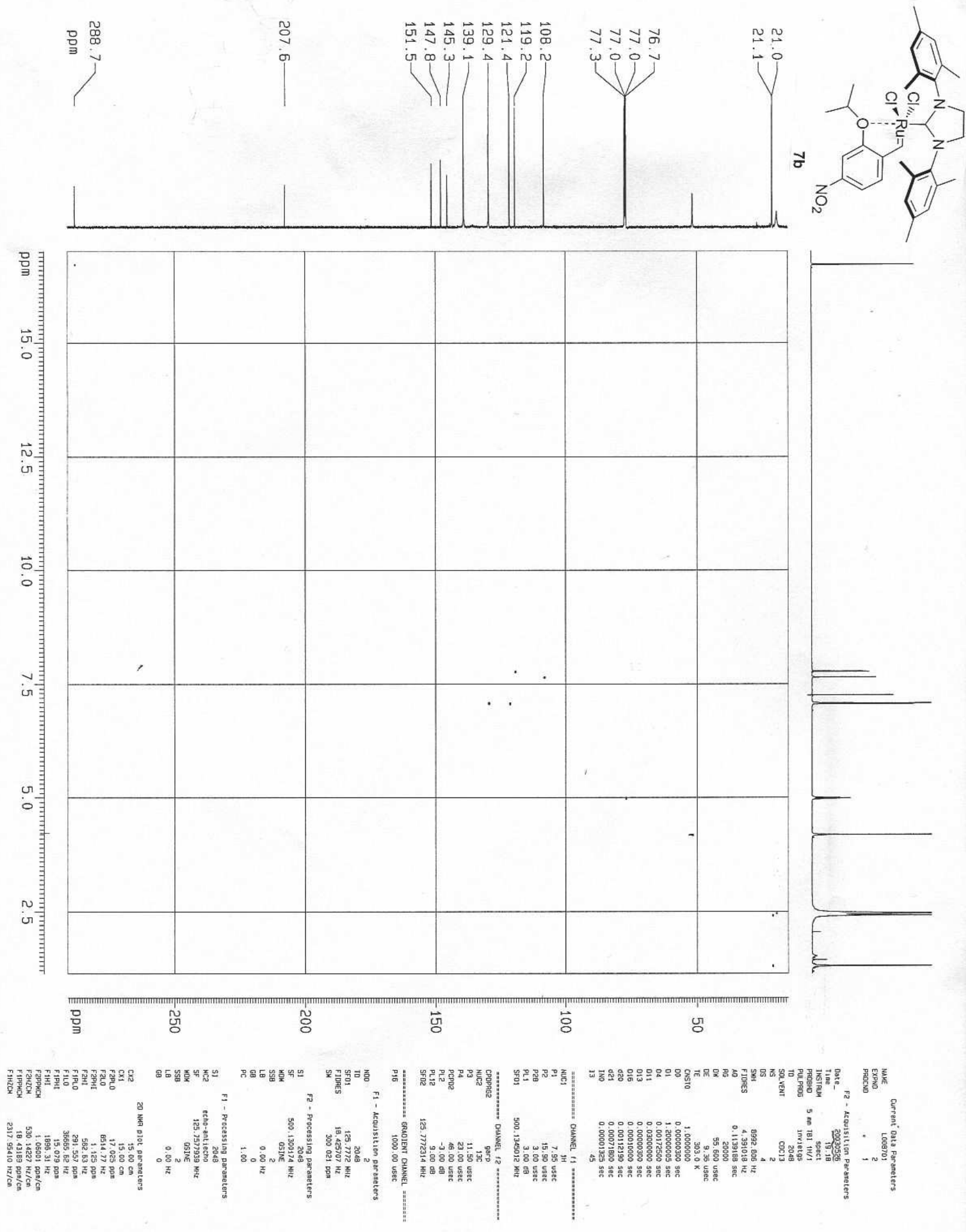




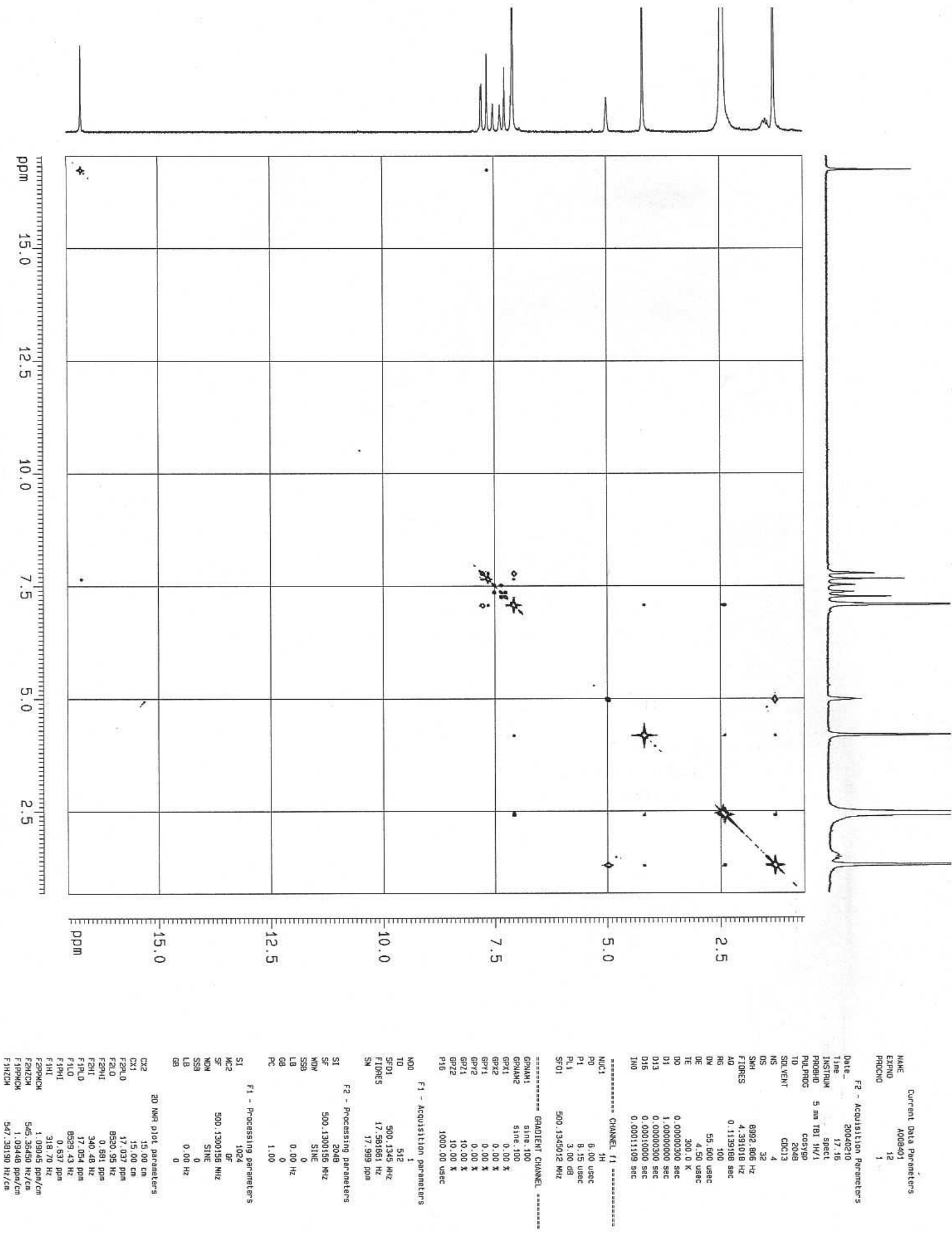




\section{Compound 14}

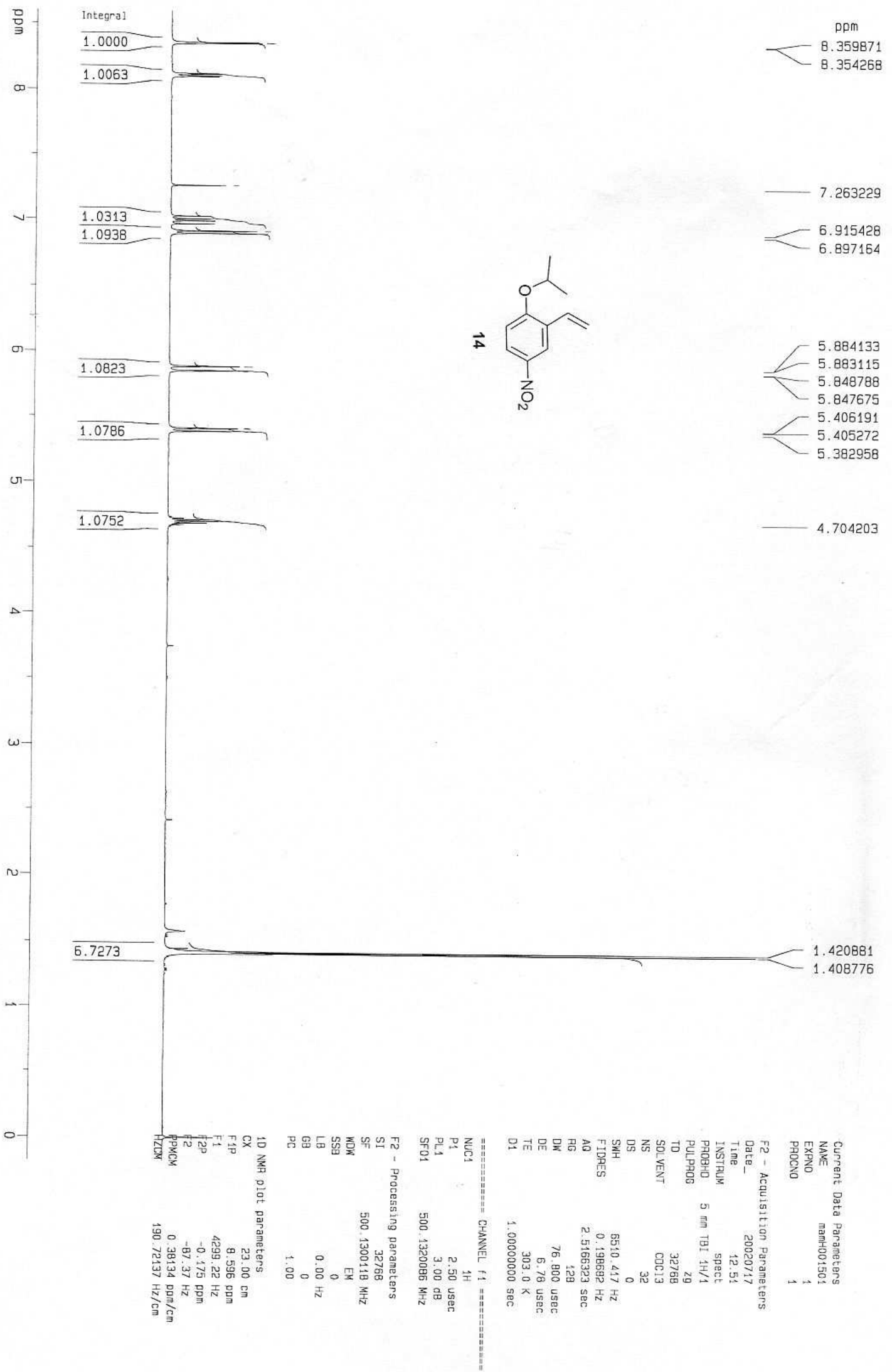




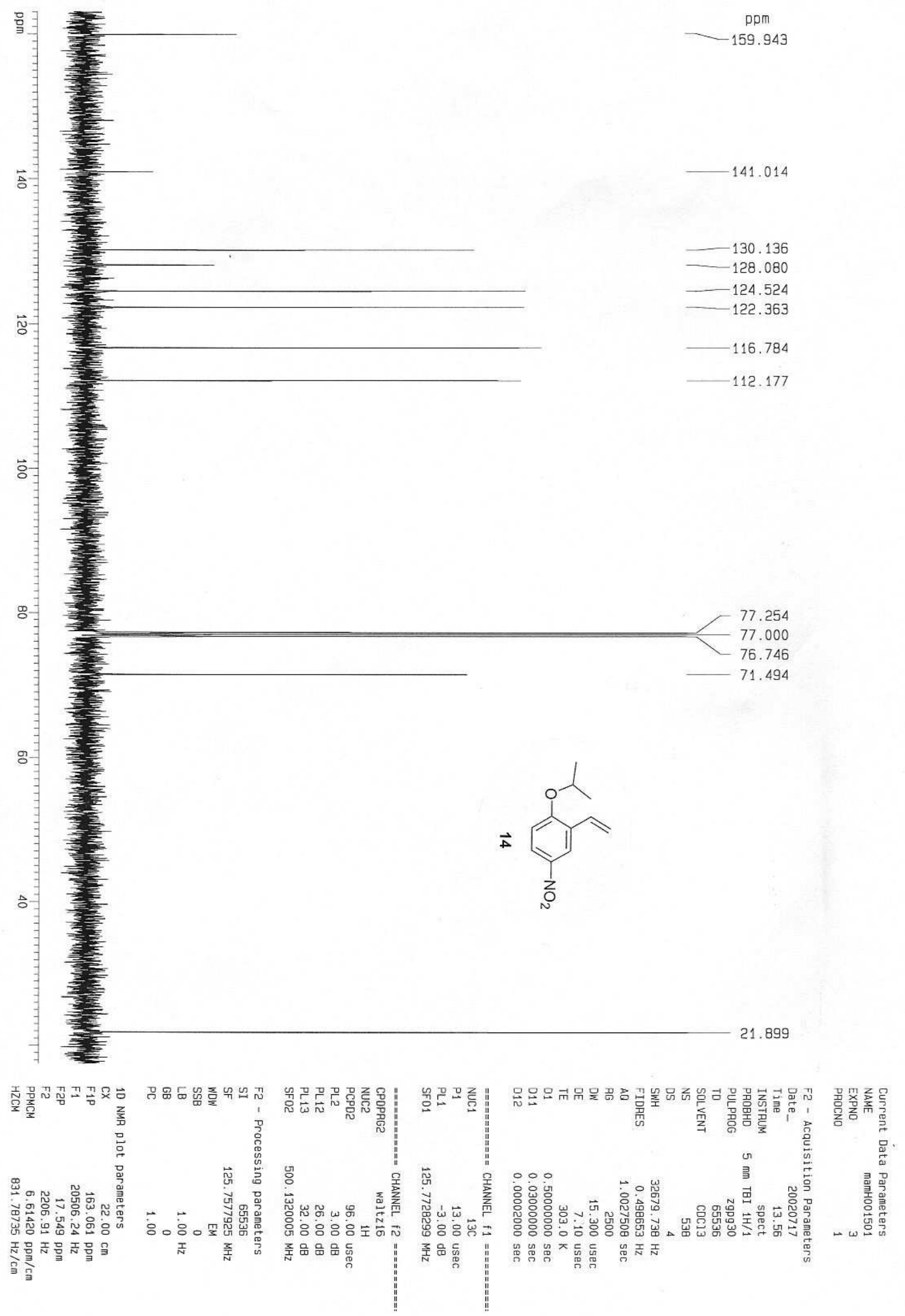




\section{Compound 15}

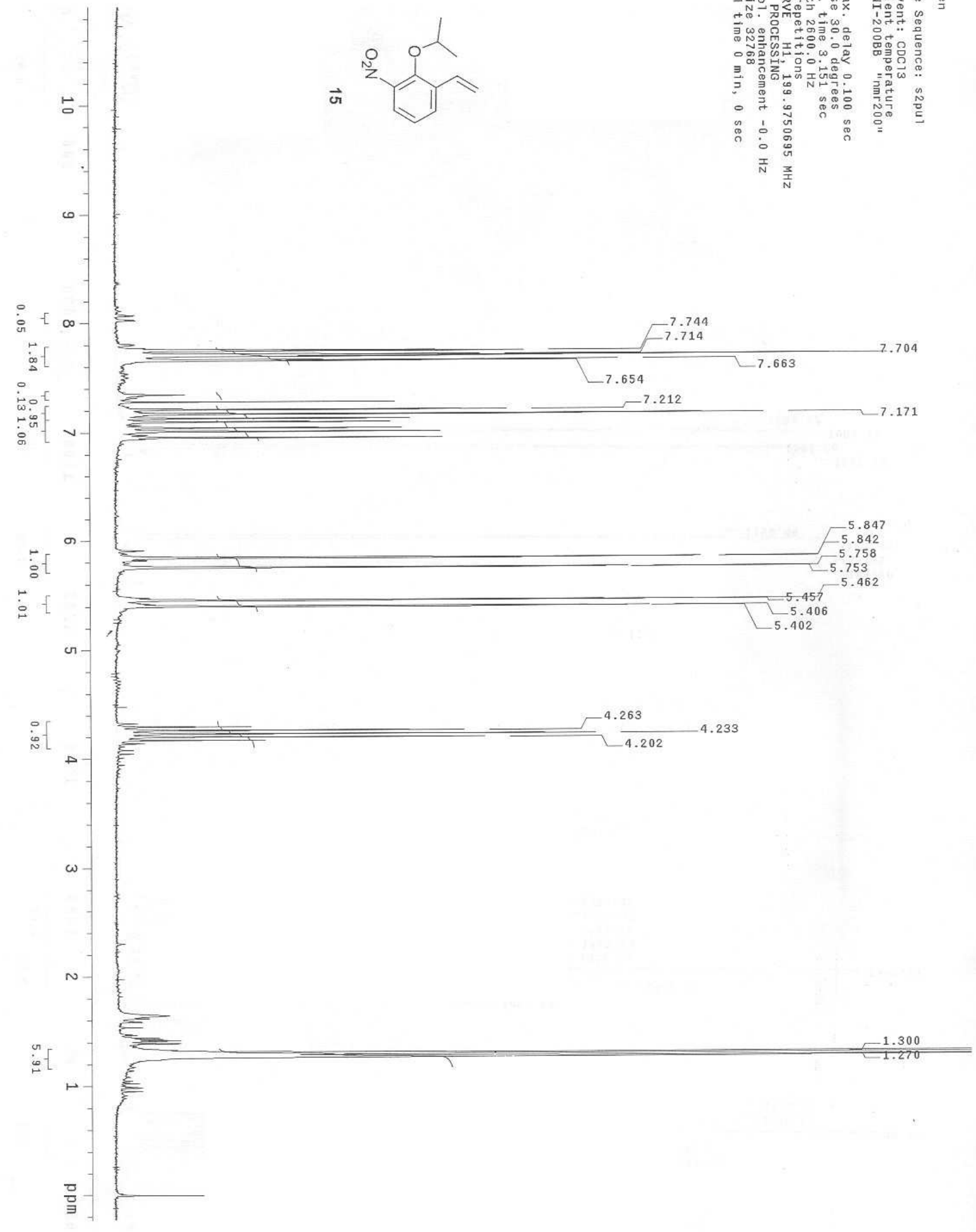




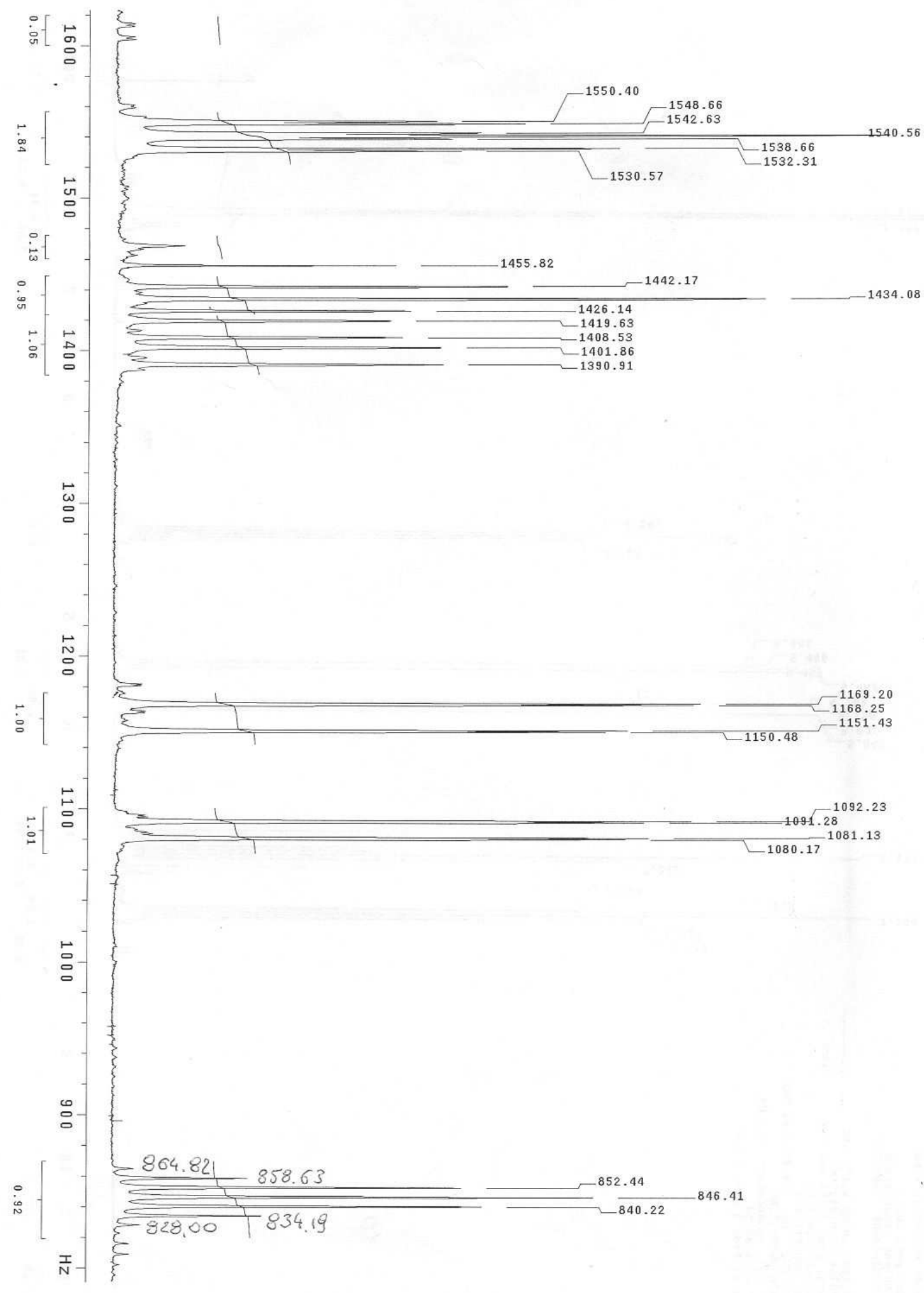




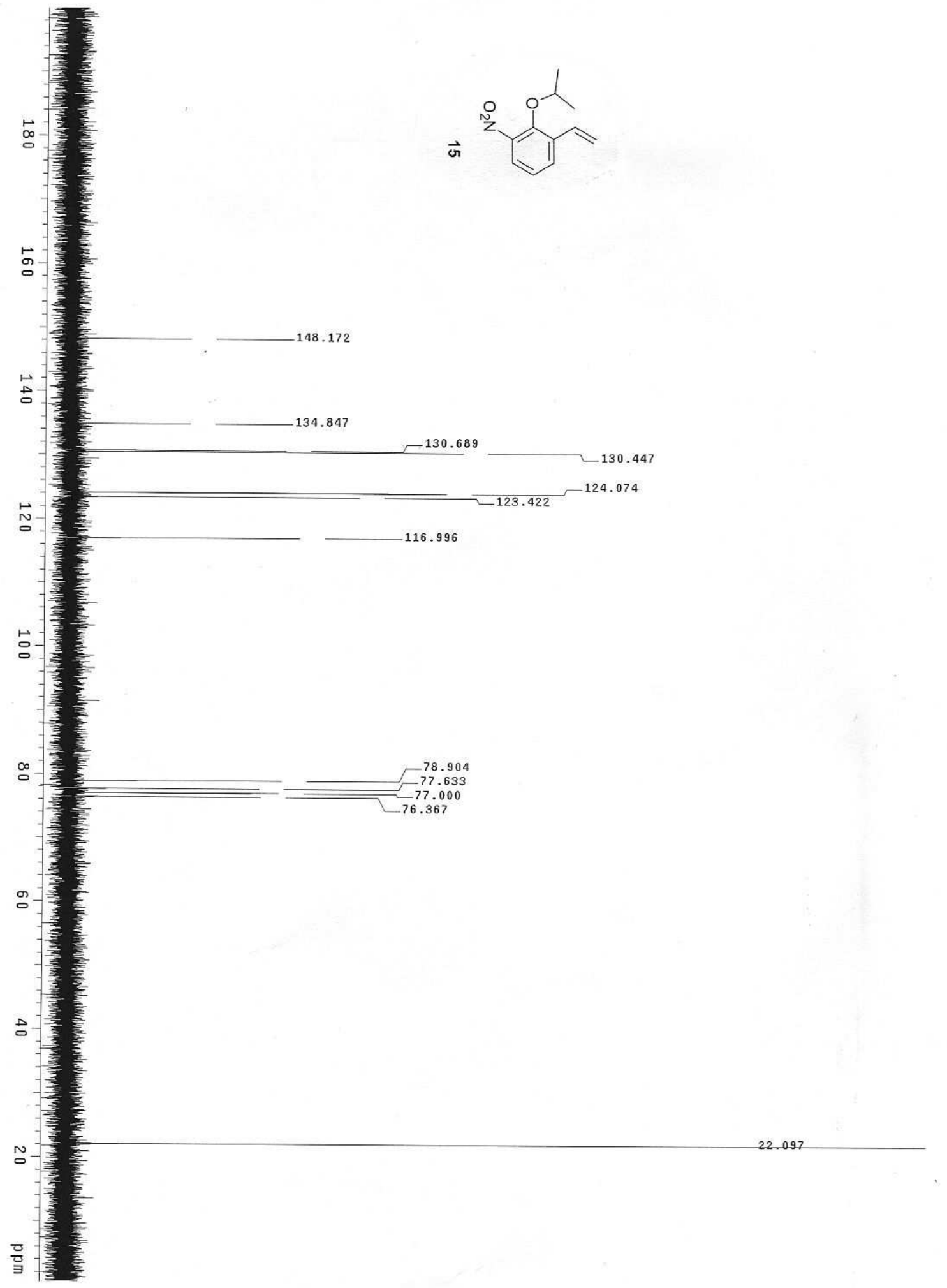




\section{Compound 16}

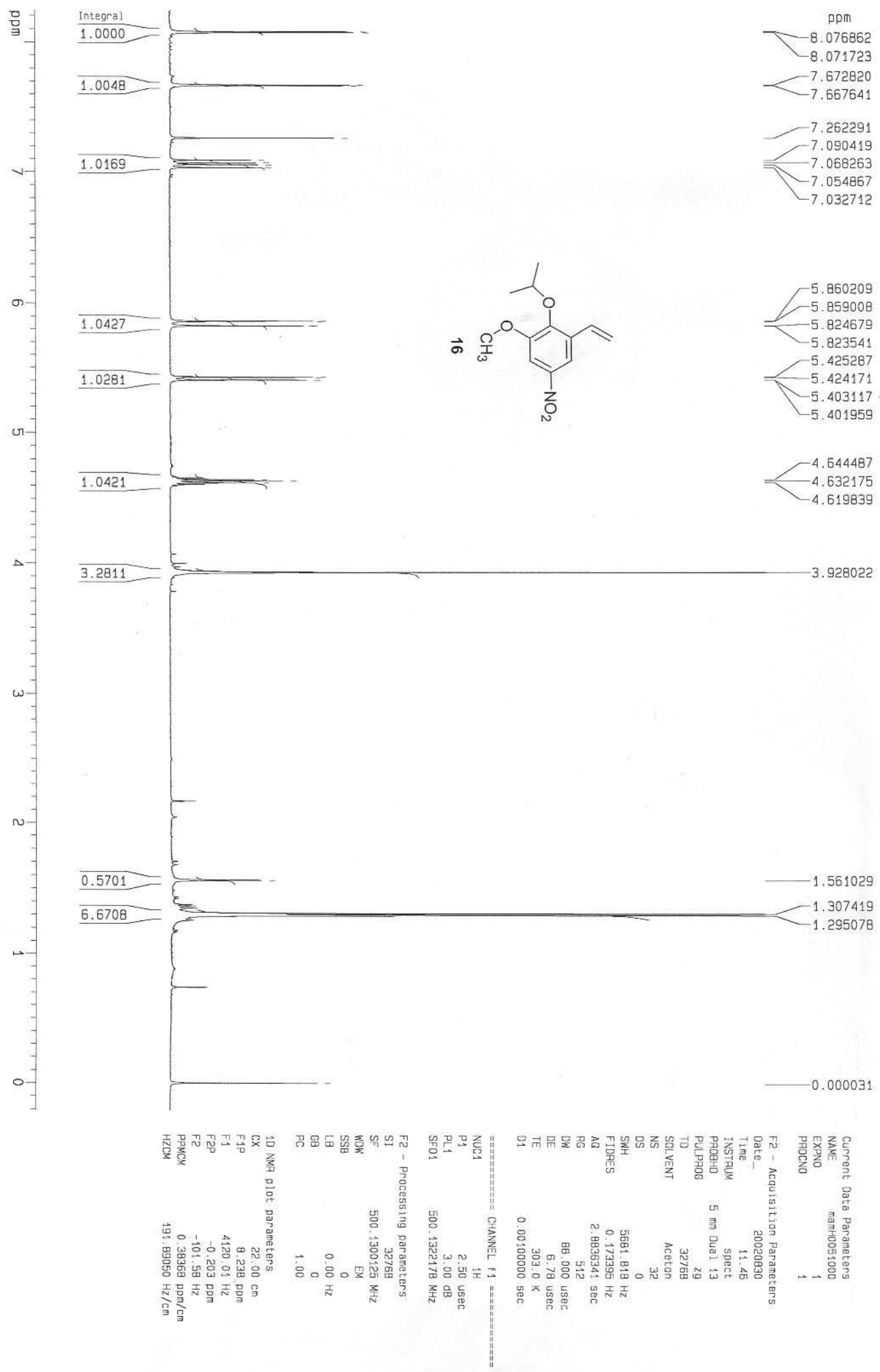



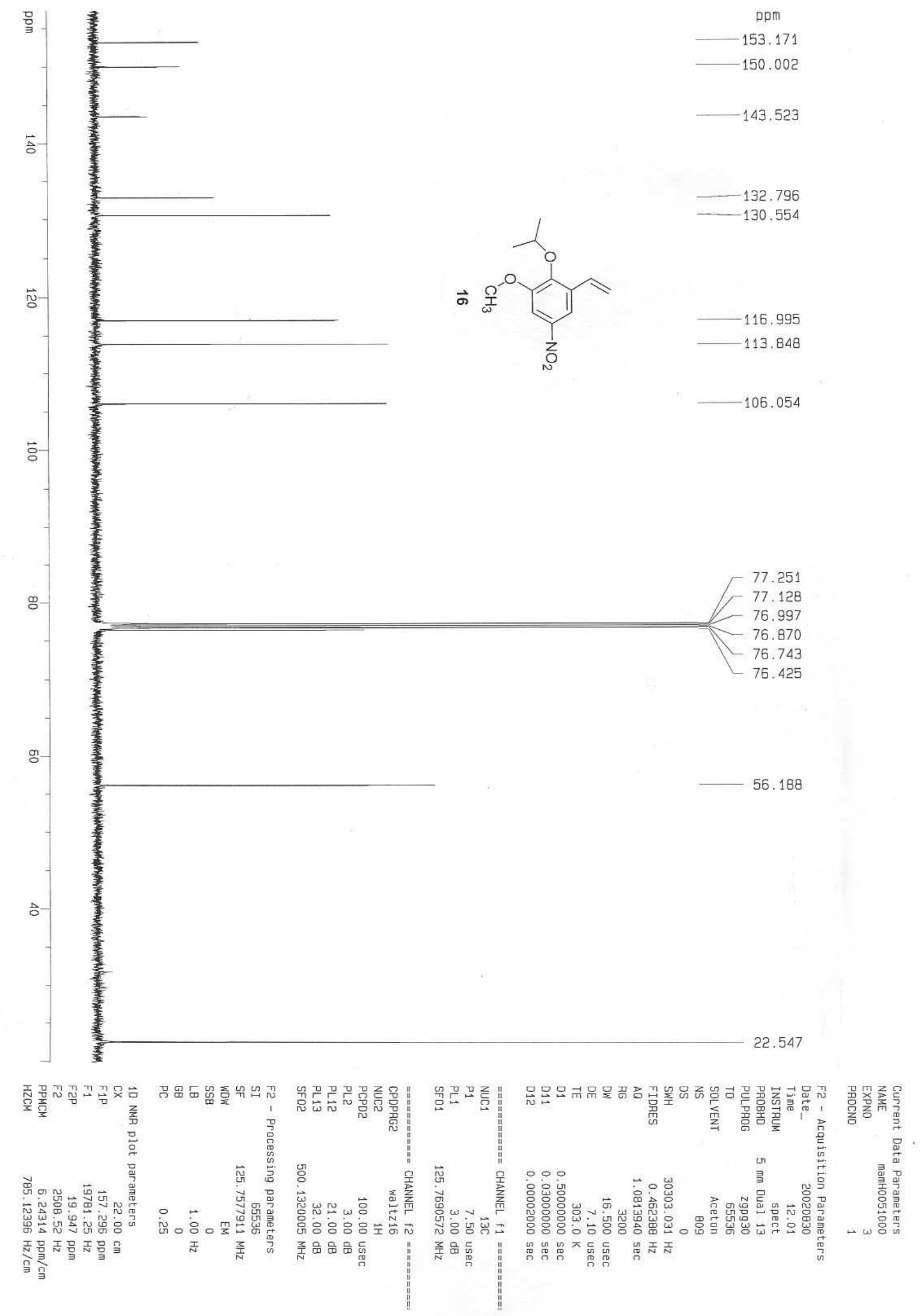


\section{Compound 19a}

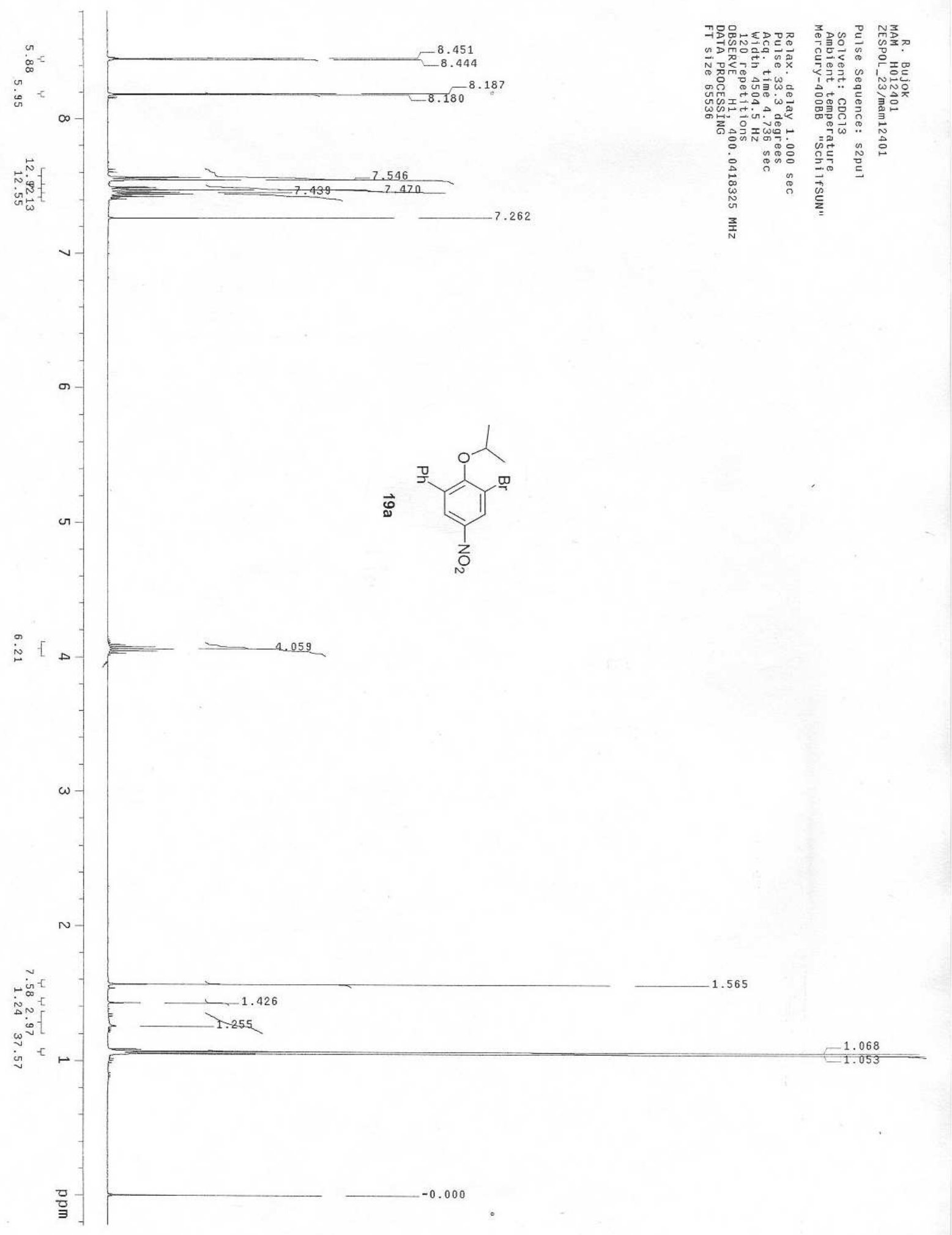




\section{Compound 20}

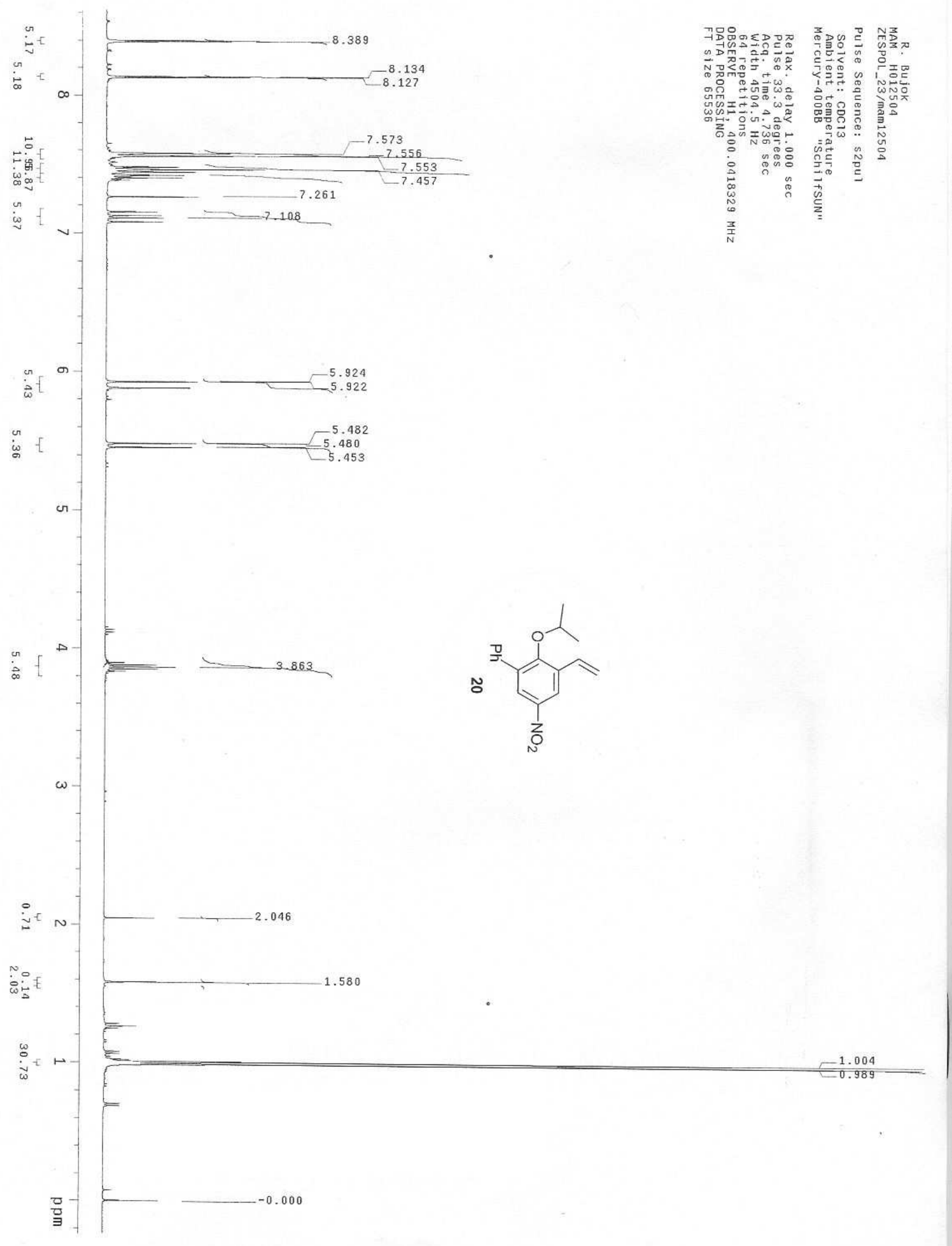




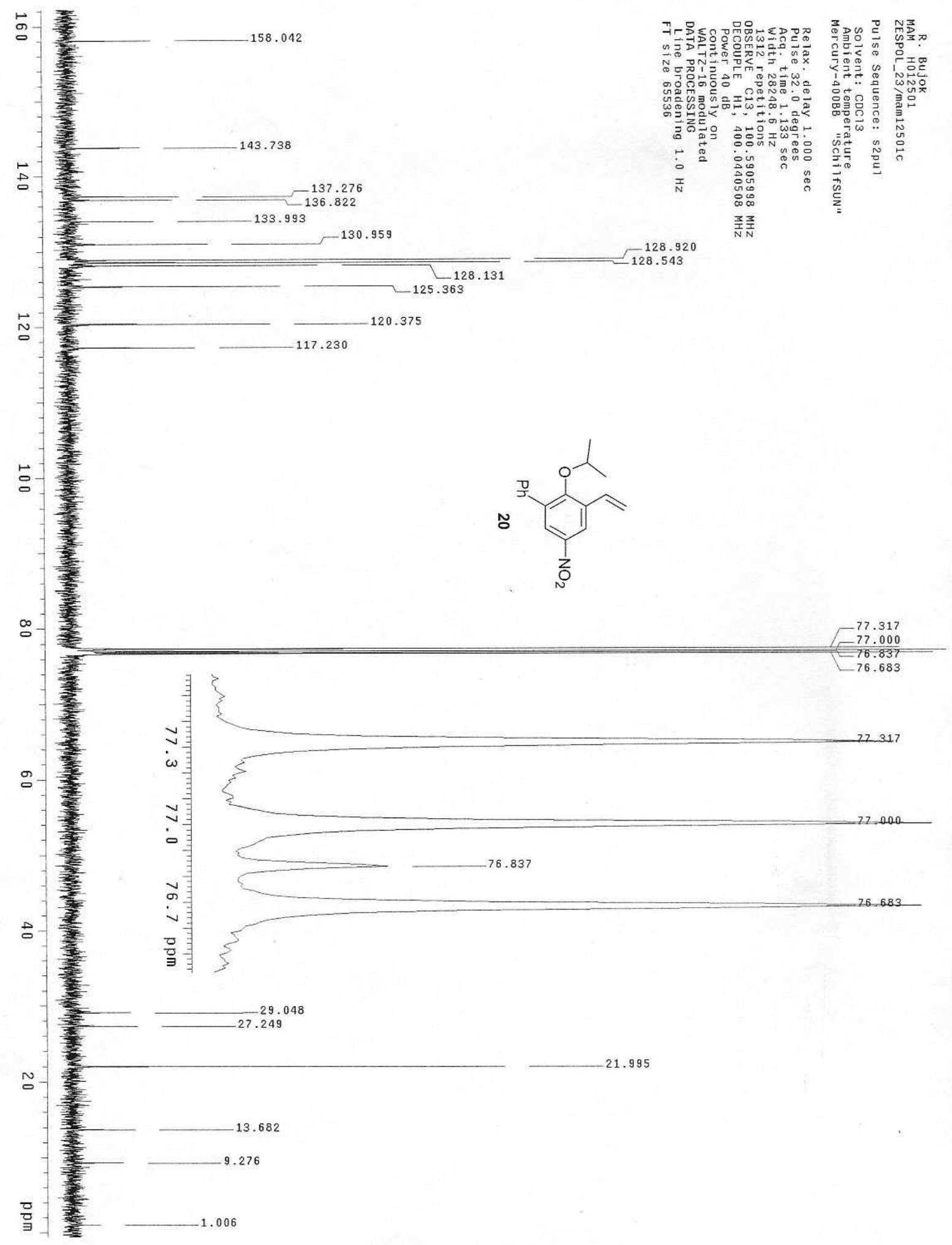




\section{Compound 22a}

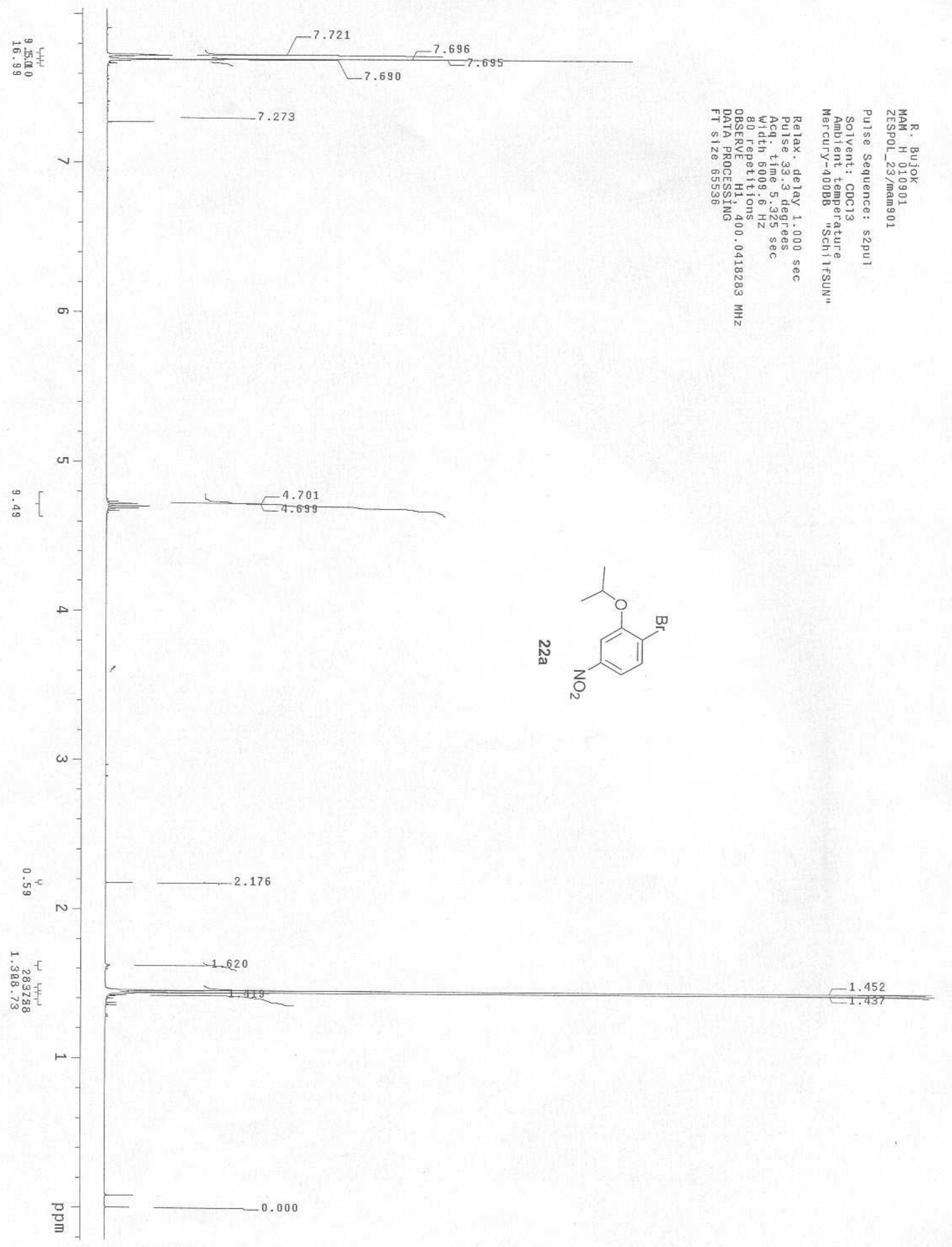




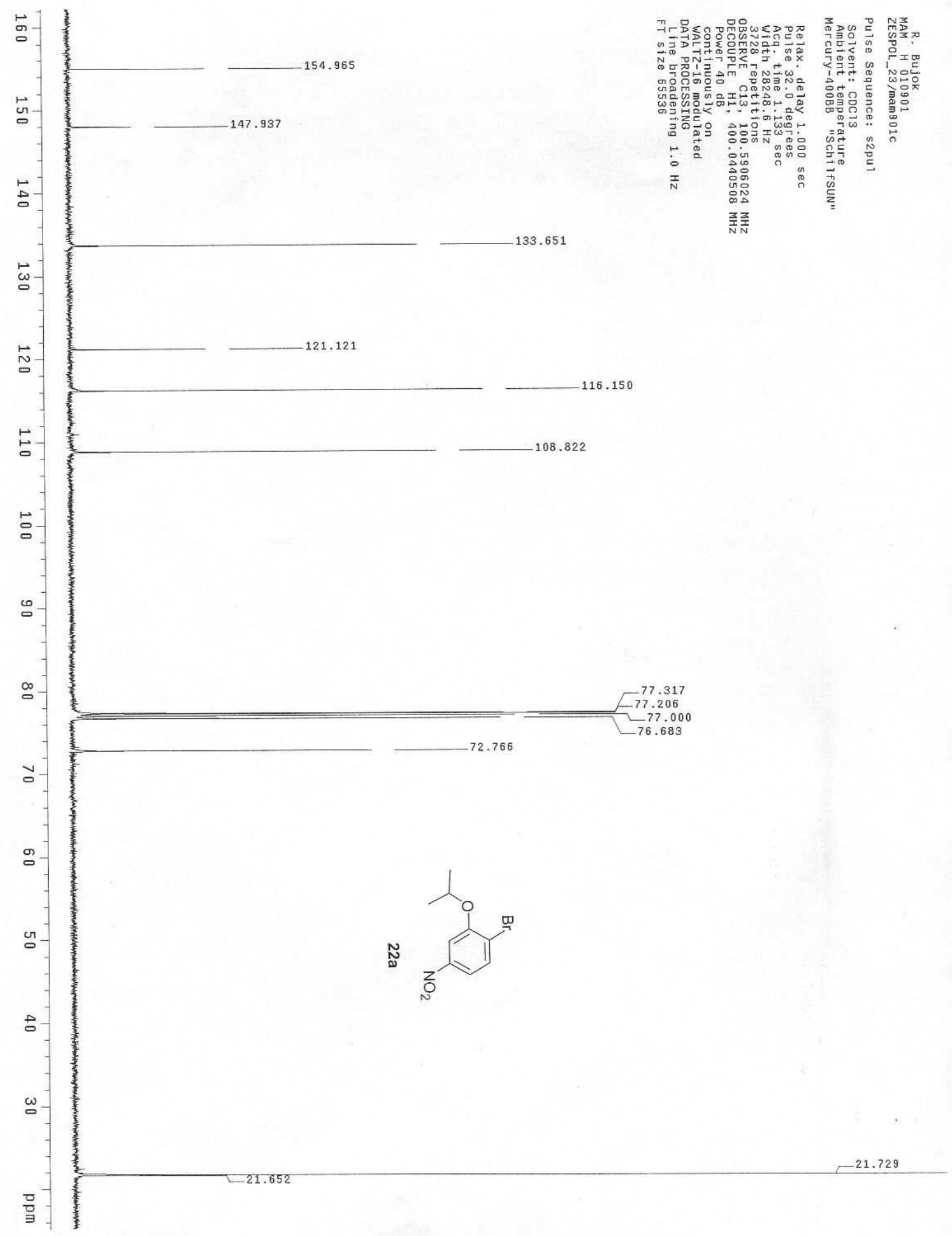




\section{Compound 23}

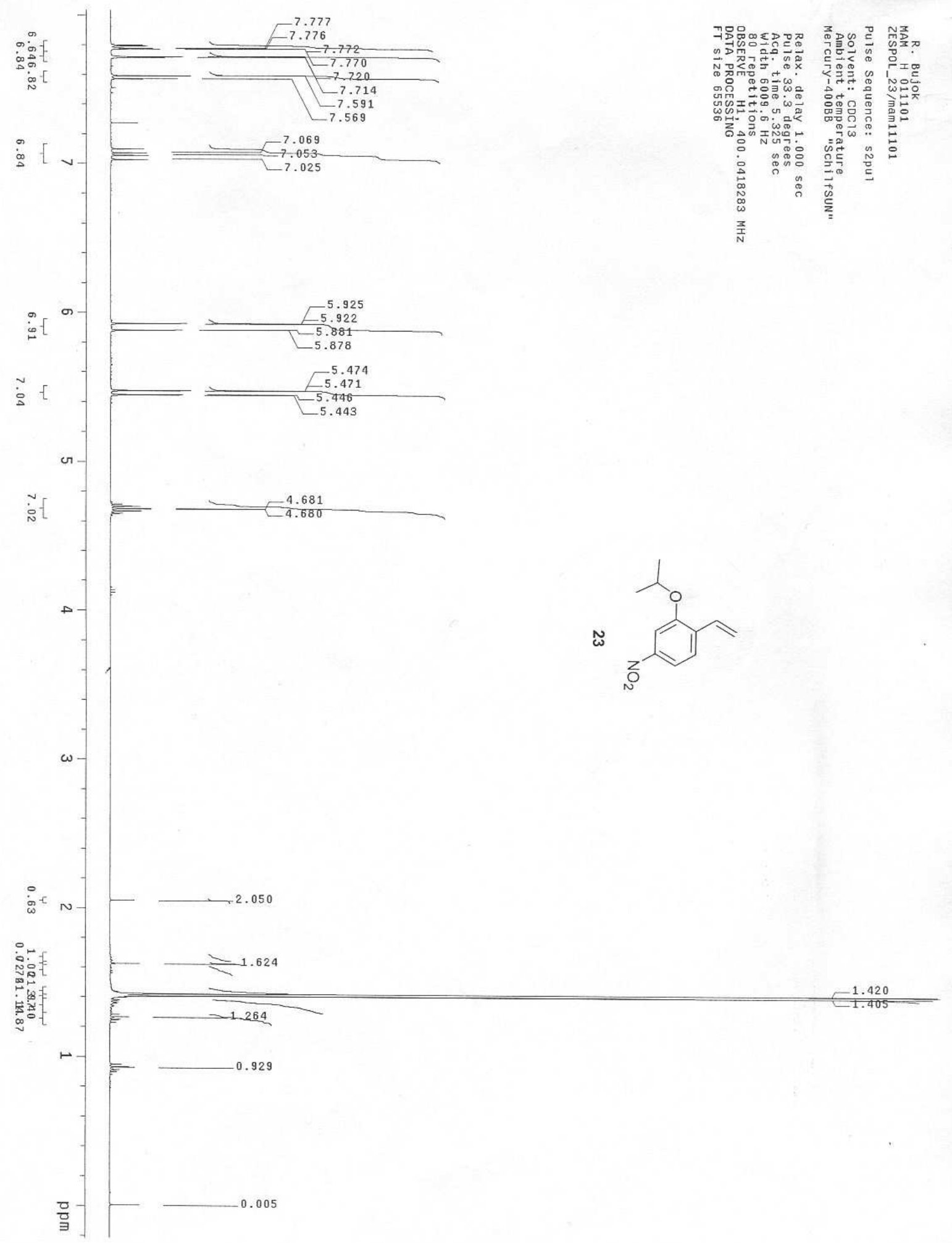




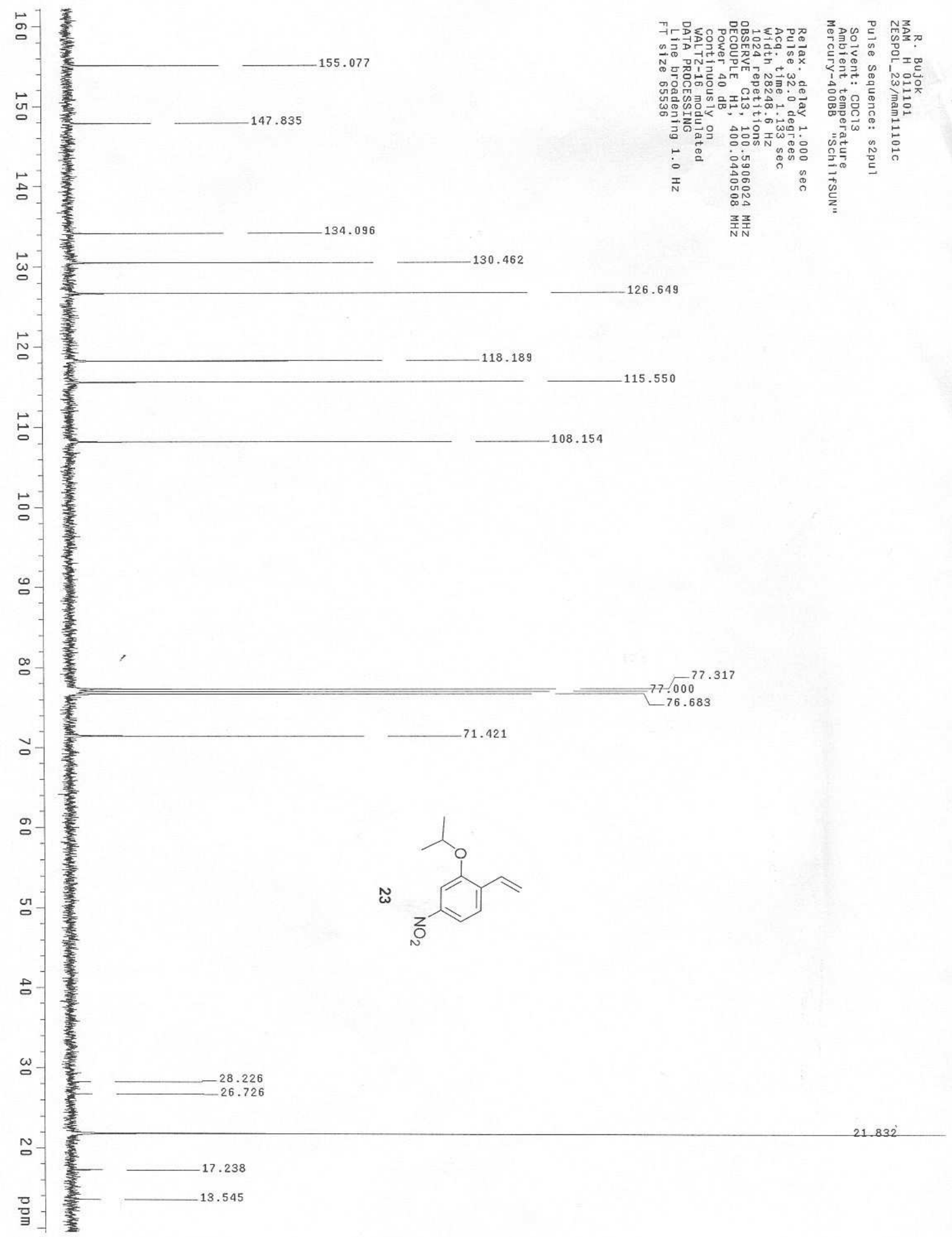




\section{Compound 70}

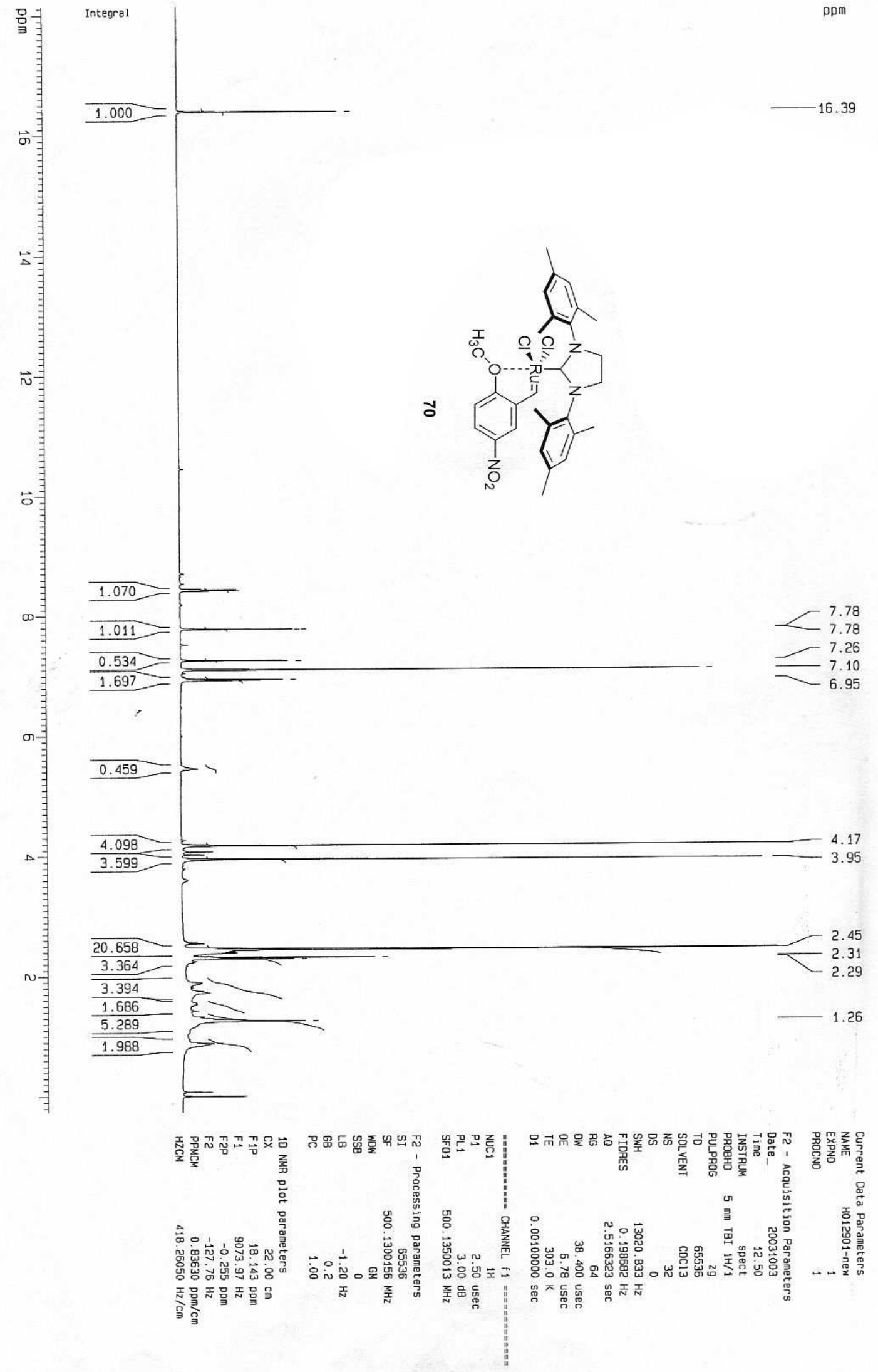




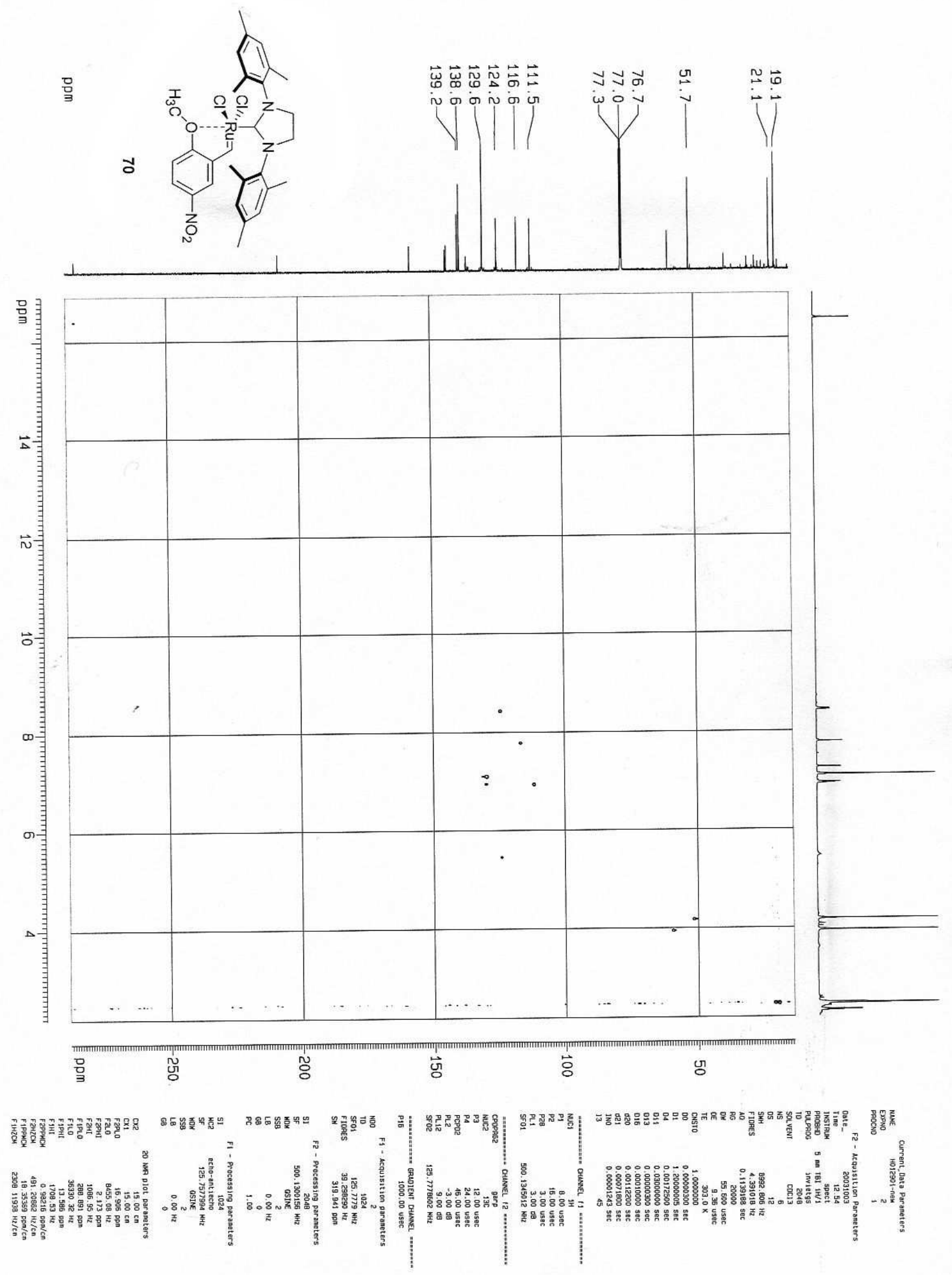




\section{Compound 70a}

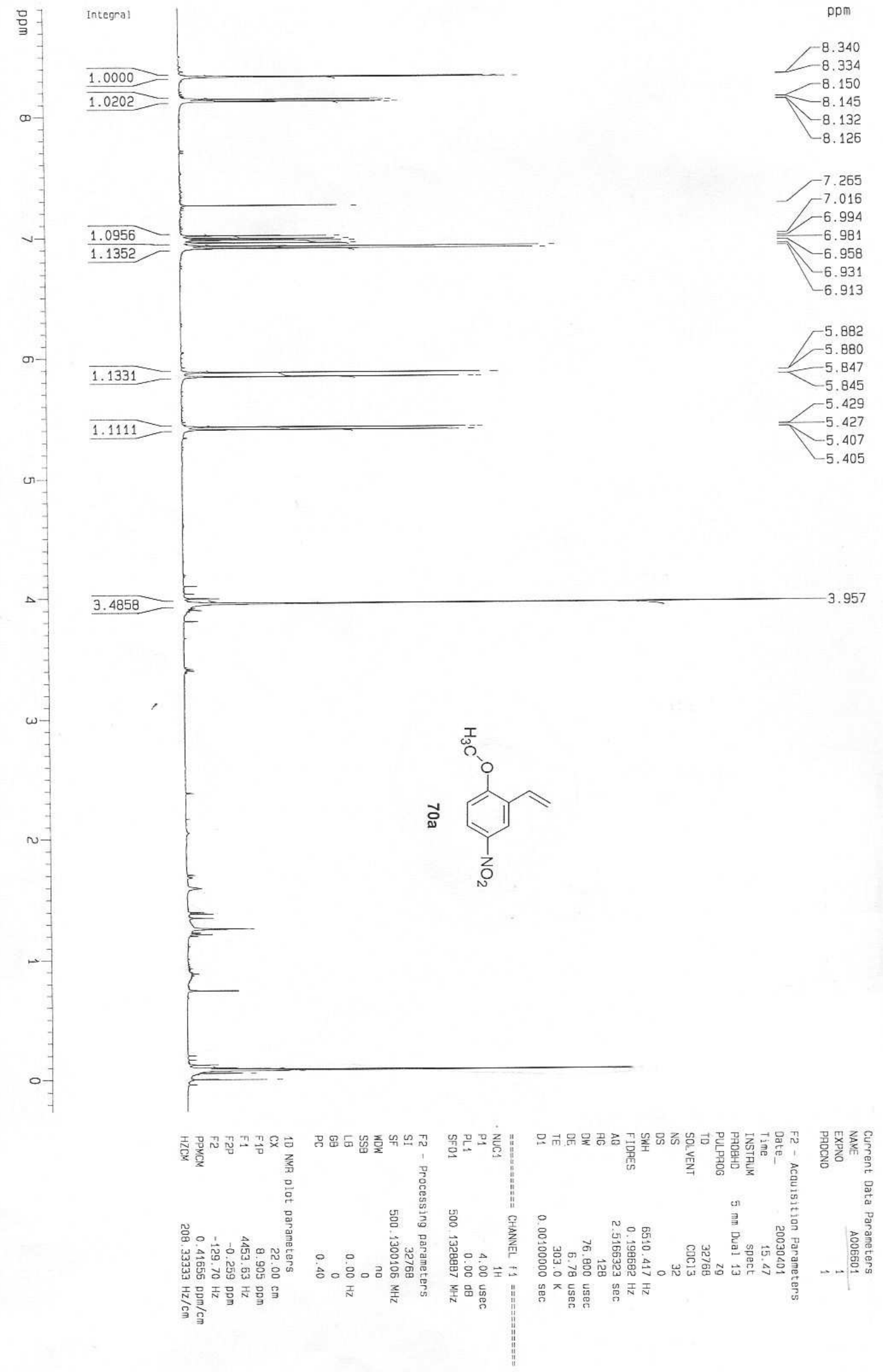




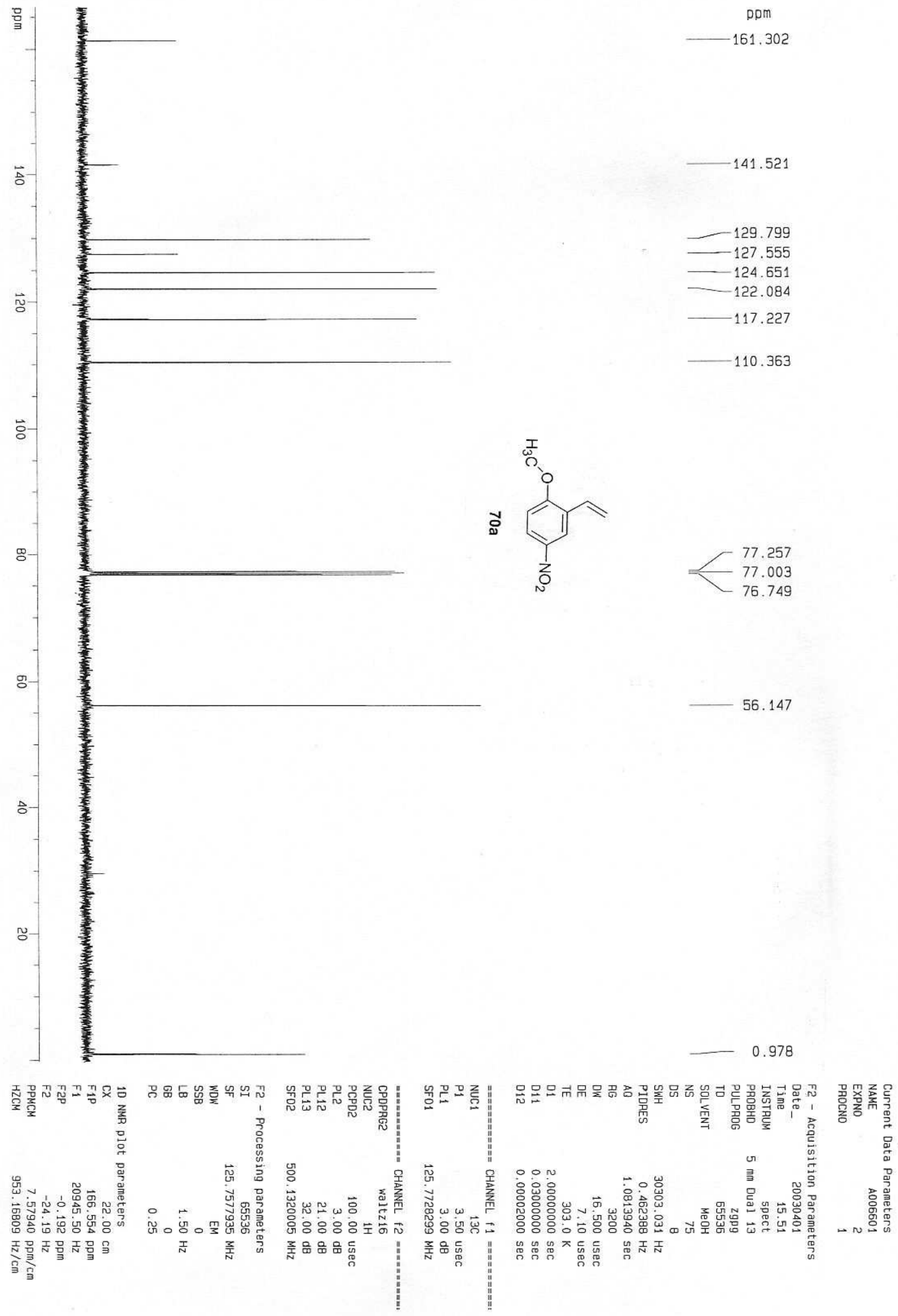

